\title{
FLPNO Nitroxide Radical Formation by a 1,1-Carboboration Route
}

\author{
Supporting Information
}

René Liedtke, ${ }^{\text {a }}$ Christina Eller, ${ }^{\text {a }}$ Constantin G. Daniliuc, ${ }^{a}$ Kamille Williams, ${ }^{b}$ Timothy H. Warren, ${ }^{\mathrm{b}}$ Matthias Tesch, ${ }^{\mathrm{a}}$ Armido Studer, ${ }^{\text {a }}$ Gerald Kehr, ${ }^{\text {a }}$ Gerhard Erker $^{\mathrm{a} *}$.

${ }^{a}$ Organisch-Chemisches Institut der Universität Münster, Corrensstraße 40, 48149 Münster, Germany

${ }^{\mathrm{b}}$ Georgetown University, 37th and O Streets NW, Washington, DC 20057-1227, United States

General Information S2

Synthesis of compound 7a S3

Synthesis of compound $\mathbf{7 b}$ S5

Synthesis of compound 7c S7

Synthesis of compound $\mathbf{8 a}$ S9

Synthesis of compound $\mathbf{8 b}$ S12

Synthesis of compound 9 S15

Synthesis of compound 12 S22

Synthesis of compound $\mathbf{1 3}$ S25

Synthesis of the diastereomers 14/14' S30

Polymerzation reactions S35 
General Information. All syntheses involving air- and moisture-sensitive compounds were carried out using standard Schlenk-type glassware (or in a glove box) under an atmosphere of argon. Solvents were dried and stored under an argon atmosphere. The following instruments were used for physical characterization of the compounds: Bruker $\operatorname{AMX} 400\left({ }^{1} \mathrm{H}: 400 \mathrm{MHz},{ }^{13} \mathrm{C}: 101 \mathrm{MHz}\right)$ Varian Inova 500 $\left({ }^{1} \mathrm{H}: 500 \mathrm{MHz},{ }^{13} \mathrm{C}: 126 \mathrm{MHz},{ }^{19} \mathrm{~F}: 470 \mathrm{MHz},{ }^{11} \mathrm{~B}: 160 \mathrm{MHz},{ }^{31} \mathrm{P}: 202 \mathrm{MHz}\right)$, Varian UnityPlus $600\left({ }^{1} \mathrm{H}\right.$ : $\left.600 \mathrm{MHz},{ }^{13} \mathrm{C}: 151 \mathrm{MHz},{ }^{19} \mathrm{~F}: 564 \mathrm{MHz},{ }^{11} \mathrm{~B}: 192 \mathrm{MHz},{ }^{31} \mathrm{P}: 243 \mathrm{MHz}\right) .{ }^{1} \mathrm{H}$ NMR and ${ }^{13} \mathrm{C}$ NMR: chemical shift $\delta$ is given relative to TMS and referenced to the solvent signal. ${ }^{19} \mathrm{~F}$ NMR: chemical shift $\delta$ is given relative to $\mathrm{CFCl}_{3}\left(\delta\left(\mathrm{CFCl}_{3}\right)=0\right.$, external reference); ${ }^{11} \mathrm{~B}$ NMR: chemical shift $\delta$ is given relative to $\mathrm{BF}_{3} \cdot \mathrm{Et}_{2} \mathrm{O}\left(\delta\left(\mathrm{BF}_{3} \cdot \mathrm{Et}_{2} \mathrm{O}\right)=0\right.$, external reference). NMR assignments are supported by additional 2D NMR experiments. Elemental analyses were performed on a Elementar Vario EI III. IR spectra were recorded on a Varian 2100 FT-IR (Excalibur Series). Melting points were obtained with a DSC Q20 (TA Instruments).

X-Ray diffraction: Data sets were collected with a Nonius KappaCCD diffractometer. Programs used: data collection, COLLECT (R. W. W. Hooft, Bruker AXS, 2008, Delft, The Netherlands); data reduction Denzo-SMN (Z. Otwinowski, W. Minor, Methods Enzymol. 1997, 276, 307-326); absorption correction, Denzo (Z. Otwinowski, D. Borek, W. Majewski, W. Minor, Acta Crystallogr. 2003, A59, 228-234); structure solution SHELXS-97 (G. M. Sheldrick, Acta Crystallogr. 1990, A46, 467-473); structure refinement SHELXL-97 (G. M. Sheldrick, Acta Crystallogr. 2008, A64, 112-122) and graphics, XP (BrukerAXS, 2000). $R$-values are given for observed reflections, and $w R^{2}$ values are given for all reflections. Exceptions and special features: For compound 8a two half dichloromethane molecules, for compound $\mathbf{9}$ one half heptane molecule, for compound $\mathbf{1 2}$ two dichloromethane molecules and for compound $\mathbf{1 3}$ one benzene molecule were found badly disordered in the asymmetric unit. The program SQUEEZE (A. L. Spek J. Appl. Cryst., 2003, 36, 7-13) was therefore used to remove mathematically the effect of the solvent. The quoted formula and derived parameters are not included the squeezed solvent molecules. For compound $\mathbf{8 a}$ one $\mathrm{SiMe}_{3}$ group and for compound $\mathbf{1 4}$ two phenyl groups are disordered over two positions. Several restraints (SADI, SAME, ISOR and SIMU) were used in order to improve refinement stability.

Materials: The following compounds were synthesized to modified literatures: a) Ethynyldi-otolylphosphane: Liedtke, R.; Kehr, G.; Fröhlich, R.; Daniliuc, C. G.; Wibbeling, B.; Petersen, J. L.; Erker, G. Helv. Chim. Acta 2012, 95, 2515; b) Ethynyldimesitylphosphane: Zhao, Y.; Lough, A. J.; Stephan, D. W. Chem. Eur. J. 2011, 17, 6731; c) [(2-bromophenyl)ethynyl]trimethylsilane (6): Swenton, J. S.; Callinan, A.; Wang, S. J. Org. Chem. 1992, 57, 78. 


\section{Syntheses of P-substituted Diynes}

\section{Synthesis of compound 7a}

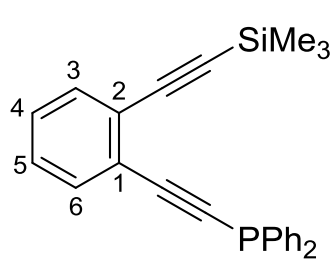

Scheme S1

1,2-Diethynylbenzene $(230 \mathrm{mg}, 1.83 \mathrm{mmol}, 1 \mathrm{eq})$ was dissolved in THF ( $20 \mathrm{~mL}$ ) and the resulting solution cooled down to $-78^{\circ} \mathrm{C}$. At this temperature a $1.6 \mathrm{M}$ solution of $n$-BuLi $(1.14 \mathrm{~mL}, 1.83 \mathrm{mmol}, 1 \mathrm{eq})$ in $n$-hexane was added slowly and the mixture was allowed to stir for $1 \mathrm{~h}$ at $-78^{\circ} \mathrm{C}$. Then $\mathrm{Ph}_{2} \mathrm{PCl}$ $(0.33 \mathrm{~mL}, 1.83 \mathrm{mmol}, 1 \mathrm{eq})$ was added and the mixture was allowed to warm to room temperature. After stirring for $2 \mathrm{~h}$ at room temperature the solvent was removed in vacuo and the residue redissolved in $\mathrm{CH}_{2} \mathrm{Cl}_{2}(\sim 50 \mathrm{~mL})$ and filtered through Celite. The solvent of the filtrate was removed in vacuo and the residue redissolved in THF $(\sim 20 \mathrm{~mL})$ and cooled to $-78^{\circ} \mathrm{C}$. At this temperature a $1.6 \mathrm{M}$ solution of $n$-BuLi $(1.14 \mathrm{~mL}, 1.83 \mathrm{mmol}, 1 \mathrm{eq})$ in $n$-hexane was added slowly and the resulting mixture was stirred for $1 \mathrm{~h}$ at $-78^{\circ} \mathrm{C}$. Then $\mathrm{CISiMe}_{3}(0.24 \mathrm{~mL}, 1.83 \mathrm{mmol}, 1 \mathrm{eq})$ was added and the mixture was allowed to warm to room temperature by stirring overnight. On the next day the solvent was removed in vacuo, the obtained residue redissolved in $\mathrm{CH}_{2} \mathrm{Cl}_{2}(\sim 50 \mathrm{~mL})$ and filtered through Celite. The solvent of the filtrate was removed in vacuo and the residue purified by flash-chromatography $\left(\mathrm{SiO}_{2}, n\right.$-pentane : $\mathrm{CH}_{2} \mathrm{Cl}_{2}=4: 1$ ) to give compound 7a $(261 \mathrm{mg}, 0.68 \mathrm{mmol}$, $37 \%)$ as a yellow oil.

IR (KBr): $\tilde{v}\left[\mathrm{~cm}^{-1}\right]=3055(w), 2961(w), 2160(w), 1476(m), 1435(m), 1250(m), 1098(m), 1027(m)$, $874(\mathrm{~s}), 844(\mathrm{~s}), 799(\mathrm{~m}), 759(\mathrm{~s}), 695(\mathrm{~s}), 647(\mathrm{w}), 512(\mathrm{~s}), 452(\mathrm{w})$.

Elemental analysis: Calcd. for $\mathrm{C}_{25} \mathrm{H}_{23}$ PSi: $\mathrm{C}, 78.50 ; \mathrm{H}, 6.06$. Found: $\mathrm{C}, 77.18 ; \mathrm{H}, 6.20$.

${ }^{1} \mathrm{H}$ NMR (500 MHz, $\left.\mathrm{CD}_{2} \mathrm{Cl}_{2}, 299 \mathrm{~K}\right): \delta=7.72(\mathrm{~m}, 4 \mathrm{H}, o-\mathrm{Ph}), 7.55(\mathrm{~m}, 1 \mathrm{H}, 6-\mathrm{H}), 7.51(\mathrm{~m}, 1 \mathrm{H}, 3-\mathrm{H}), 7.37$ (m, 6H, m,p-Ph), $7.32(\mathrm{~m}, 2 \mathrm{H}, 4,5-\mathrm{H}), 0.16\left(\mathrm{~s}^{2}{ }^{2} \mathrm{~J}_{\mathrm{SiH}}=7.1 \mathrm{~Hz}, 9 \mathrm{H}, \mathrm{SiCH}_{3}\right)$.

${ }^{13} \mathrm{C}\left\{{ }^{1} \mathrm{H}\right\}$ NMR (126 MHz, CD ${ }_{2} \mathrm{Cl}$, $\left.299 \mathrm{~K}\right): \delta=136.7\left(\mathrm{~d},{ }^{1} \mathrm{JPC}_{\mathrm{PC}}=6.8 \mathrm{~Hz}, i-\mathrm{Ph}\right), 133.0\left(\mathrm{~d},{ }^{2} J_{\mathrm{PC}}=21.2 \mathrm{~Hz}, o-\mathrm{Ph}\right)$, $132.8(\mathrm{C} 3), 132.6\left(\mathrm{~d},{ }^{4} J_{\mathrm{PC}}=1.6 \mathrm{~Hz}, \mathrm{C} 6\right), 129.5\left(\mathrm{~d},{ }^{4} J_{\mathrm{PC}}=0.6 \mathrm{~Hz}, p-\mathrm{Ph}\right), 129.06\left(\mathrm{~d},{ }^{3} \mathrm{JPC}_{\mathrm{PC}}=7.7 \mathrm{~Hz}, m-\mathrm{Ph}\right)$, 129.05 (C4), 128.7 (C5), 126.1 (d, $\left.{ }^{4} \mathrm{~J}_{\mathrm{PC}}=1.8 \mathrm{~Hz}, \mathrm{C} 2\right), 125.6$ (d, $\left.{ }^{3} \mathrm{JPC}_{\mathrm{PC}}=1.3 \mathrm{~Hz}, \mathrm{C} 1\right), 106.6\left(\mathrm{~d},{ }^{2} \mathrm{JPC}_{\mathrm{PC}}=3.9 \mathrm{~Hz}\right.$, $\left.{ }^{\mathrm{C} 1} \mathrm{C} \equiv\right), 103.4\left({ }^{2} J_{\mathrm{SiC}}=15.8 \mathrm{~Hz},{ }^{\mathrm{C}} \mathrm{C} \equiv\right), 99.6\left({ }^{1} J_{\mathrm{SiC}}=81.4 \mathrm{~Hz}, \equiv \mathrm{CSi}\right), 90.2\left(\mathrm{~d},{ }^{1} J_{\mathrm{PC}}=8.2 \mathrm{~Hz}, \equiv \mathrm{CP}\right),-0.1\left({ }^{1} J_{\mathrm{SiC}}=\right.$ $\left.56.4 \mathrm{~Hz}, \mathrm{SiCH}_{3}\right)$.

${ }^{31} \mathrm{P}\left\{{ }^{1} \mathrm{H}\right\}$ NMR (202 MHz, $\left.\mathrm{CD}_{2} \mathrm{Cl}_{2}, 299 \mathrm{~K}\right): \delta=-33.3\left(\mathrm{v}_{1 / 2} \sim 2 \mathrm{~Hz}\right)$.

${ }^{31} \mathrm{P}$ NMR (202 MHz, $\mathrm{CD}_{2} \mathrm{Cl}_{2}, 299 \mathrm{~K}$ ): $\delta=-33.3$ (quin $\mathrm{m},{ }^{3} \mathrm{~J}_{\mathrm{PH}} \sim 8 \mathrm{~Hz}$ ).

${ }^{29} \mathrm{Si}\left\{{ }^{1} \mathrm{H}\right\}$ DEPT NMR $\left(99 \mathrm{MHz}, \mathrm{CD}_{2} \mathrm{Cl}_{2}, 299 \mathrm{~K}\right): \delta=-17.3\left(\mathrm{v}_{1 / 2} \sim 2 \mathrm{~Hz}\right)$. 


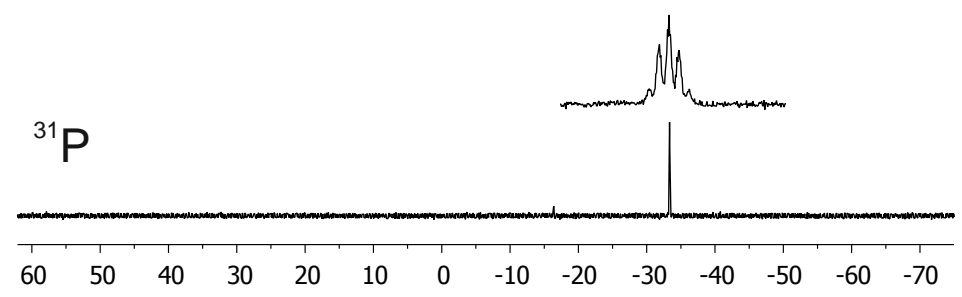

\section{$\mathrm{SiCH}_{3}$}
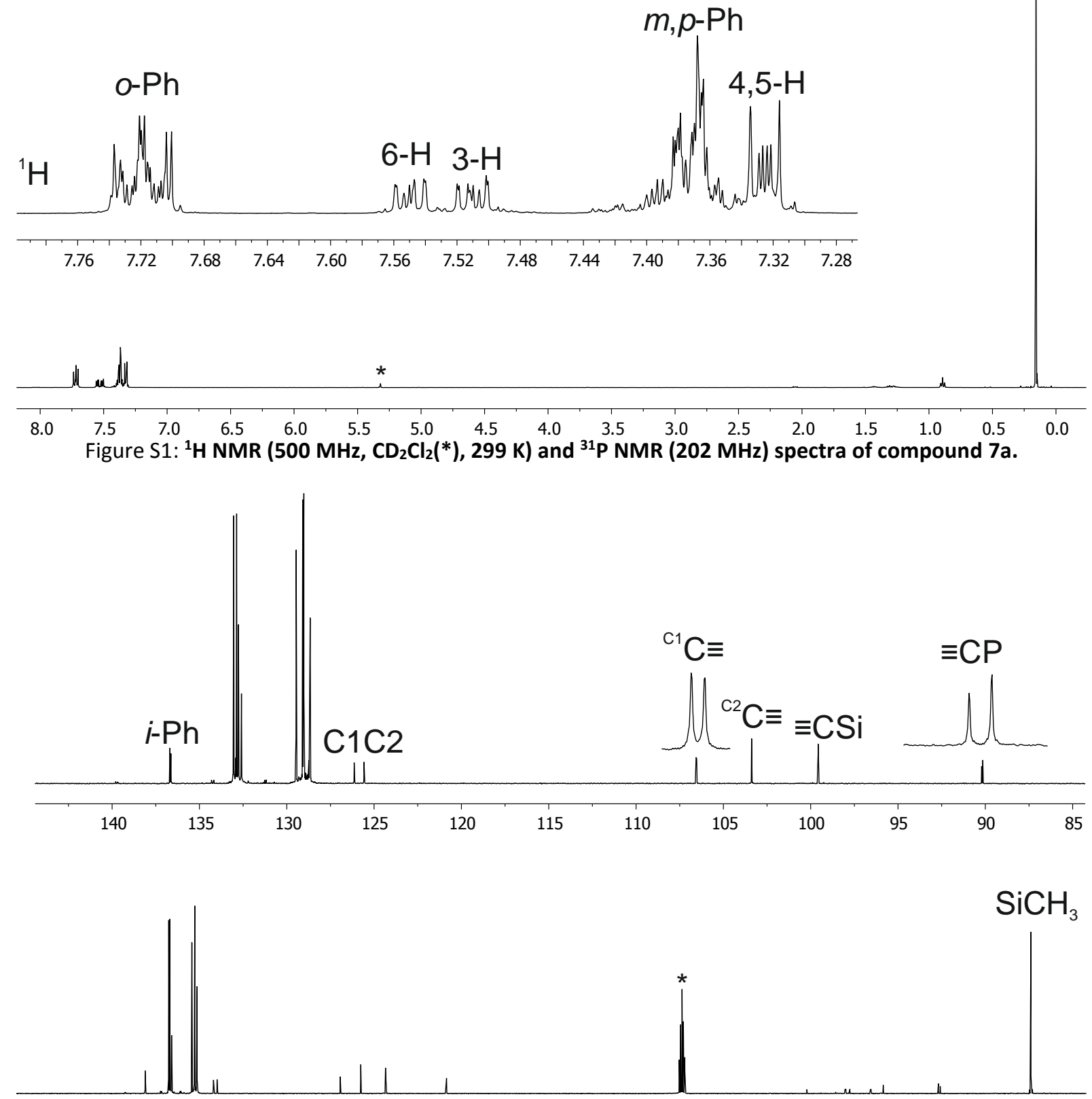

$\mathrm{SiCH}_{3}$

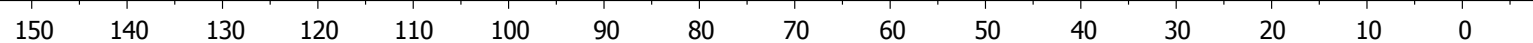
Figure S2: ${ }^{13} \mathrm{C}\left\{{ }^{1} \mathrm{H}\right\}$ NMR $\left(126 \mathrm{MHz}, \mathrm{CD}_{2} \mathrm{Cl}_{2}\left({ }^{*}\right), 299 \mathrm{~K}\right)$ spectrum of compound $7 \mathrm{a}$. 


\section{Synthesis of compound $7 \mathrm{~b}$}

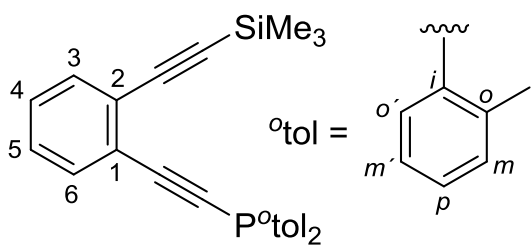

Scheme S2

Ethynyldi-o-tolylphosphane $(1.3 \mathrm{~g}, 5.46 \mathrm{mmol}, 1.2 \mathrm{eq})$ was dissolved in THF $(\sim 10 \mathrm{~mL})$. The solution was cooled to $0{ }^{\circ} \mathrm{C}$ and at this temperature a $1.6 \mathrm{M}$ solution of $n$-BuLi $(3.41 \mathrm{~mL}$, $5.46 \mathrm{mmol}, 1.2 \mathrm{eq})$ in $n$-hexane was added. The resulting mixture was stirred for $1 \mathrm{~h}$ at $0{ }^{\circ} \mathrm{C}$. Then a solution of $\mathrm{ZnCl}_{2}$ (744 mg, $5.46 \mathrm{mmol}, 1.2 \mathrm{eq}$, dried at $110^{\circ} \mathrm{C}$ overnight) in THF $(\sim 10 \mathrm{~mL}$ ) was added and the resulting reaction mixture was allowed to warm to room temperature. The mixture was stirred for $30 \mathrm{~min}$ and then a mixture of [(2-bromophenyl)ethynyl]trimethylsilane $\left(6,1.15 \mathrm{~g}, 4.55 \mathrm{mmol}, 1\right.$ eq) and $\mathrm{Pd}\left(\mathrm{PPh}_{3}\right)_{4}$ (636 mg, $0.55 \mathrm{mmol}, 10 \mathrm{~mol} \%)$ in toluene $(\sim 10 \mathrm{~mL})$ was added. The resulting reaction mixture was stirred for $3 \mathrm{~d}$ at $80^{\circ} \mathrm{C}$. After cooling to room temperature a $1 \mathrm{M}$ solution of $\mathrm{HCl}(\sim 100 \mathrm{~mL})$ was added and subsequently the phases were separated. The aqueous phase was extracted with $\mathrm{Et}_{2} \mathrm{O}(3 \mathrm{x}$ $\sim 50 \mathrm{~mL})$ and the combined organic phases were washed with brine $(\sim 50 \mathrm{~mL})$ and $\mathrm{H}_{2} \mathrm{O}(\sim 50 \mathrm{~mL})$. Then the organic phase was dried with $\mathrm{MgSO}_{4}$ and the solvent was removed in vacuo. The residue was purified by flash-chromatography ( $n$-pentane : $\mathrm{CH}_{2} \mathrm{Cl}_{2}=7: 1$ ) to give compound $7 \mathbf{b}$ (490 $\mathrm{mg}$, $1.19 \mathrm{mmol}, 26 \%$ ) as a yellow oil.

IR (KBr): $\tilde{v}\left[\mathrm{~cm}^{-1}\right]=3057(\mathrm{~m}), 3006(\mathrm{w}), 2959(\mathrm{~m}), 2159(\mathrm{~m}), 1589(\mathrm{w}), 1473(\mathrm{~m}), 1452(\mathrm{~m}), 1379(\mathrm{w})$, $1249(m), 1229(w), 1201(w), 1160(w), 1131(w), 1098(w), 1036(w), 949(w), 875(s), 842(s), 798$ $(\mathrm{m}), 750(\mathrm{~s}), 715(\mathrm{~s}), 646(\mathrm{~m}), 553(\mathrm{~m}), 523(\mathrm{~s}), 453(\mathrm{~s})$.

Elemental analysis: Calcd. for $\mathrm{C}_{27} \mathrm{H}_{27} \mathrm{PSi}$ : $\mathrm{C}, 78.99 ; \mathrm{H}, 6.63$. Found: $\mathrm{C}, 78.98 ; \mathrm{H}, 6.34$.

${ }^{1} \mathrm{H}$ NMR (500 MHz, $\left.\mathrm{CD}_{2} \mathrm{Cl}_{2}, 299 \mathrm{~K}\right): \delta=7.63\left(\mathrm{~m}, 2 \mathrm{H}, o^{\prime}-^{\circ} \mathrm{tol}\right), 7.50(\mathrm{~m}, 1 \mathrm{H}, 6-\mathrm{H}), 7.48(\mathrm{~m}, 1 \mathrm{H}, 3-\mathrm{H}), 7.30$ $\left(\mathrm{m}, 4 \mathrm{H}, p^{\circ}{ }^{\circ}\right.$ tol $\left., 4,5-\mathrm{H}\right), 7.22\left(\mathrm{~m}, 2 \mathrm{H}, m^{-}{ }^{\circ} \mathrm{tol}\right), 7.21\left(\mathrm{~m}, 2 \mathrm{H}, m^{\prime}-{ }^{\circ} \mathrm{tol}\right), 2.50\left(\mathrm{~m}, 6 \mathrm{H}, o-\mathrm{CH}_{3}{ }^{\mathrm{tol}}\right), 0.16\left(\mathrm{~s}^{2}{ }^{2} \mathrm{~J}_{\mathrm{SiH}}=\right.$ $7.1 \mathrm{~Hz}, 9 \mathrm{H}, \mathrm{SiCH}_{3}$ ).

${ }^{13} \mathrm{C}\left\{{ }^{1} \mathrm{H}\right\}$ NMR $\left(126 \mathrm{MHz}, \mathrm{CD}_{2} \mathrm{Cl}, 299 \mathrm{~K}\right): \delta=142.3\left(\mathrm{~d},{ }^{2} J_{\mathrm{PC}}=27.2 \mathrm{~Hz}, \mathrm{o}^{-}{ }^{\circ}\right.$ tol $), 133.42\left(\mathrm{~d},{ }^{2} \mathrm{JPC}_{\mathrm{PC}}=3.3 \mathrm{~Hz}\right.$, $o^{\prime}{ }^{\circ}{ }^{\circ}$ tol), 133.37 (d, ${ }^{1} J_{\mathrm{PC}}=6.2 \mathrm{~Hz}, i^{\circ}{ }^{\circ}$ tol), $132.8(\mathrm{C} 3), 132.7$ (d, $\left.{ }^{4} J_{\mathrm{PC}}=1.9 \mathrm{~Hz}, \mathrm{C} 6\right), 130.6\left(\mathrm{~d},{ }^{3} \mathrm{JPC}_{\mathrm{PC}}=5.3 \mathrm{~Hz}\right.$, $m$ - $^{\circ}$ tol), 129.7 (p- ${ }^{\circ}$ tol), 128.9 (C4), 128.6 (C5), 126.7 (d, ${ }^{3} J_{\mathrm{PC}}=2.3 \mathrm{~Hz}, m^{\prime}-{ }^{\circ}$ tol), 125.9 (d, ${ }^{4} \mathrm{JCC}_{\mathrm{PC}}=1.9 \mathrm{~Hz}$, C2), $125.7\left(d,{ }^{3} J_{P C}=1.5 \mathrm{~Hz}, C 1\right), 106.6\left(d,{ }^{2} J_{P C}=4.1 \mathrm{~Hz},{ }^{C 1} C \equiv\right), 103.4\left({ }^{C 2} \mathrm{C} \equiv\right), 99.4$ (三CSi), 89.9 (d, ${ }^{1} J_{P C}=9.3$ $\mathrm{Hz}, \equiv C P), 21.4\left(\mathrm{~d}^{3} \mathrm{JPC}_{\mathrm{PC}}=20.9 \mathrm{~Hz}, \mathrm{o}-\mathrm{CH}_{3}{ }^{\mathrm{tol}}\right),-0.1\left({ }^{1} \mathrm{JSC}_{\mathrm{SiC}}=56.2 \mathrm{~Hz}, \mathrm{SiCH}_{3}\right)$.

${ }^{31} \mathrm{P}\left\{{ }^{1} \mathrm{H}\right\}$ NMR (202 MHz, $\left.\mathrm{CD}_{2} \mathrm{Cl}_{2}, 299 \mathrm{~K}\right): \delta=-47.4\left(\mathrm{v}_{1 / 2} \sim 1 \mathrm{~Hz}\right)$.

${ }^{29} \mathrm{Si}\left\{{ }^{1} \mathrm{H}\right\}$ DEPT (99 MHz, $\left.\mathrm{CD}_{2} \mathrm{Cl}_{2}, 299 \mathrm{~K}\right): \delta=-17.4\left(\mathrm{v}_{1 / 2} \sim 2 \mathrm{~Hz}\right)$. 


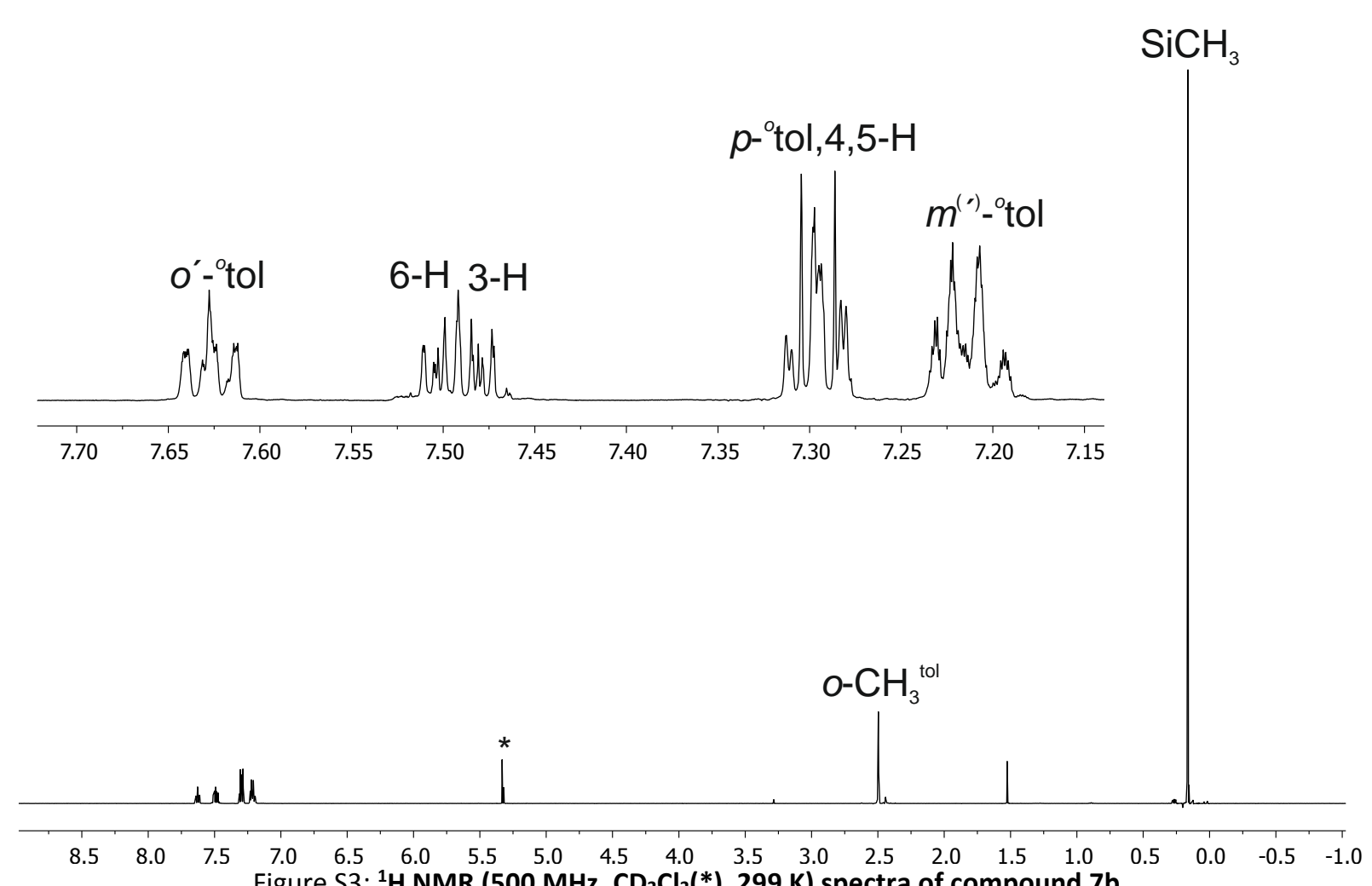
Figure S3: ${ }^{1} \mathrm{H}$ NMR (500 MHz, $\mathrm{CD}_{2} \mathrm{Cl}_{2}(*)$, $299 \mathrm{~K}$ ) spectra of compound 7b.
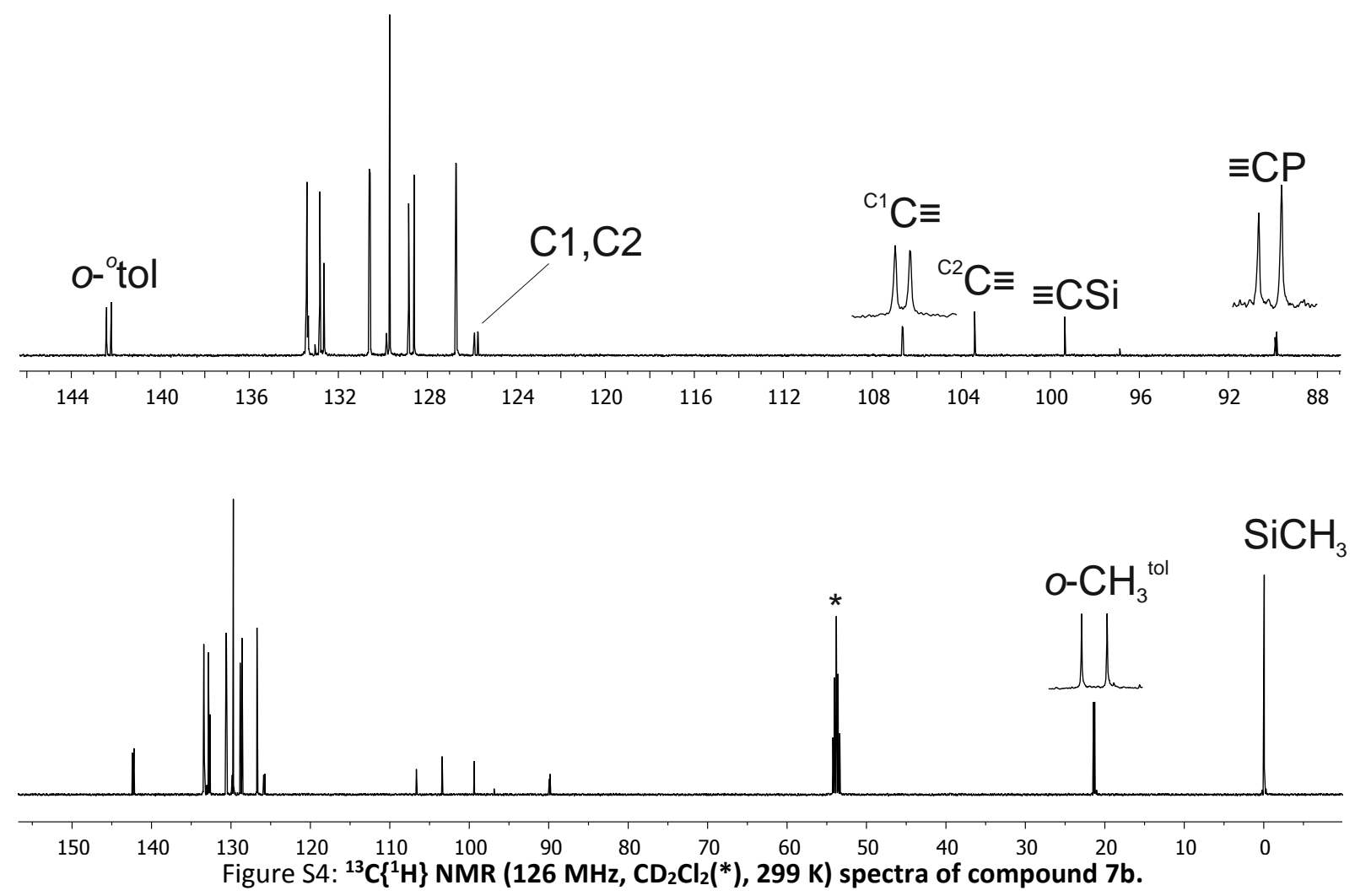


\section{Synthesis of compound 7c}

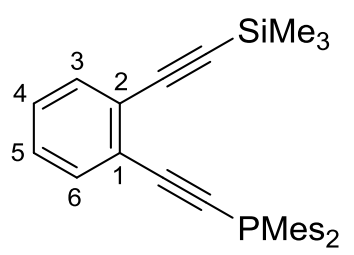

Scheme S3

Ethynyldimesitylphosphane (1.2 g, $4.1 \mathrm{mmol}, 1.2 \mathrm{eq})$ was dissolved in THF ( $10 \mathrm{~mL}$ ) and the resulting solution cooled to $0{ }^{\circ} \mathrm{C}$. At this temperature a $1.6 \mathrm{M}$ solution of $n$-BuLi $(2.6 \mathrm{~mL}, 4.1 \mathrm{mmol}, 1.2 \mathrm{eq})$ in $n$-hexane was added dropwise. After complete addition the resulting mixture was allowed to warm to room temperature and stirred for $1 \mathrm{~h}$ at this temperature. Then the solution was cooled to $0{ }^{\circ} \mathrm{C}$ again and a solution of $\mathrm{ZnCl}_{2}(556 \mathrm{mg}, 4.1 \mathrm{mmol}, 1.2 \mathrm{eq})$ in THF ( $10 \mathrm{~mL}$ ) was added at this temperature. The mixture was allowed to warm to room temperature and stirred for $30 \mathrm{~min}$. To this reaction mixture a solution of [(2-bromophenyl)ethynyl]trimethylsilane (6, $897 \mathrm{mg}, 3.42 \mathrm{mmol}, 1 \mathrm{eq})$ in toluene $(\sim 20 \mathrm{~mL})$ and $\mathrm{Pd}\left(\mathrm{PPh}_{3}\right)_{4}(392 \mathrm{mg}, 0.41 \mathrm{mmol}, 10 \mathrm{~mol} \%)$ was added. The resulting mixture was stirred at $80^{\circ} \mathrm{C}$ for $5 \mathrm{~d}$. After cooling to room temperature a $1 \mathrm{M}$ aqueous solution of $\mathrm{HCl}(\sim 100 \mathrm{~mL})$ was added and the phases were separated. The aqueous phase was extracted with $\mathrm{Et}_{2} \mathrm{O}(3 \mathrm{x} \sim 50 \mathrm{~mL})$ and the combined organic phases were washed with brine ( $50 \mathrm{~mL})$ and $\mathrm{H}_{2} \mathrm{O}(\sim 50 \mathrm{~mL})$. After drying with $\mathrm{MgSO}_{4}$ and removing the solvent in vacuo the crude product was purified by flash-chromatography $\left(\mathrm{SiO}_{2}, n\right.$-pentane : $\left.\mathrm{CH}_{2} \mathrm{Cl}_{2}=8: 1\right)$ to give compound 7c $(1.20 \mathrm{~g}, 2.67 \mathrm{mmol}, 75 \%)$ as a yellow oil.

IR (KBr): $\tilde{v}\left[\mathrm{~cm}^{-1}\right]=2959(\mathrm{br}), 2158(\mathrm{~m}), 1602(\mathrm{w}), 1558(\mathrm{w}), 1473(\mathrm{~m}), 1451(\mathrm{~m}), 1377(\mathrm{w}), 1248(\mathrm{~m})$, $1202(w), 1159(w), 1098 w), 1035(w), 875(s), 884(s), 755(s), 714(w), 647(w), 619(w), 554(w)$, $512(w)$.

Elemental analysis: Calcd. for $\mathrm{C}_{31} \mathrm{H}_{35}$ PSi: $\mathrm{C}, 79.79 ; \mathrm{H}, 7.56$. Found: $\mathrm{C}, 79.73 ; \mathrm{H}, 7.55$

${ }^{1} \mathrm{H}$ NMR (500 MHz, $\left.\mathrm{CD}_{2} \mathrm{Cl}_{2}, 299 \mathrm{~K}\right): \delta=7.44(\mathrm{~m}, 1 \mathrm{H}, 3-\mathrm{H}), 7.37(\mathrm{~m}, 1 \mathrm{H}, 6-\mathrm{H}), 7.25(\mathrm{~m}, 1 \mathrm{H}, 5-\mathrm{H}), 7.24(\mathrm{~m}$, $1 \mathrm{H}, 4-\mathrm{H}), 6.83\left(\mathrm{dm},{ }^{4} J_{\mathrm{PH}}=3.3 \mathrm{~Hz}, 4 \mathrm{H}, m-\mathrm{Mes}\right), 2.43\left(\mathrm{~s}, 12 \mathrm{H}, o-\mathrm{CH}_{3}{ }^{\text {Mes }}\right), 2.25\left(\mathrm{~s}, 6 \mathrm{H}, p-\mathrm{CH}_{3}{ }^{\text {Mes }}\right), 0.19$ (s, $\left.{ }^{2} J_{\text {SiH }}=7.7 \mathrm{~Hz}, 9 \mathrm{H}, \mathrm{SiCH}_{3}\right)$.

${ }^{13} \mathrm{C}\left\{{ }^{1} \mathrm{H}\right\}$ NMR (126 MHz, $\left.\mathrm{CD}_{2} \mathrm{Cl}_{2}, 299 \mathrm{~K}\right): \delta=142.4$ (d, $\left.{ }^{2} \mathrm{JPC}_{\mathrm{PC}}=15.6 \mathrm{~Hz}, \mathrm{o}-\mathrm{Mes}\right), 138.9$ (p-Mes), 133.0 (C3), $132.3\left(\mathrm{~d},{ }^{4} \mathrm{~J}_{\mathrm{PC}}=2.4 \mathrm{~Hz}, \mathrm{C} 6\right), 130.2\left(\mathrm{~d},{ }^{3} \mathrm{~J}_{\mathrm{PC}}=3.8 \mathrm{~Hz}, m-\mathrm{Mes}\right), 129.8$ (d, $\left.{ }^{1} J_{\mathrm{PC}}=11.8 \mathrm{~Hz}, i-\mathrm{Mes}\right), 128.5$ (C5), $128.4(C 4), 126.3\left(d,{ }^{3} J_{P C}=1.6 \mathrm{~Hz}, C 1\right), 125.4\left(d,{ }^{4} J_{P C}=2.4 \mathrm{~Hz}, C 2\right), 105.5$ (d, $\left.{ }^{2} J_{\mathrm{PC}}=7.9 \mathrm{~Hz},{ }^{\mathrm{C}}{ }^{1} \mathrm{C} \equiv\right), 103.6$ $\left({ }^{\mathrm{C} 2} \mathrm{C} \equiv\right), 98.8(\equiv \mathrm{CSi}), 92.1\left(\mathrm{~d},{ }^{1} J_{\mathrm{PC}}=8.8 \mathrm{~Hz}, \equiv \mathrm{CP}\right), 23.4\left(\mathrm{~d},{ }^{3} J_{\mathrm{PC}}=14.3 \mathrm{~Hz}, o-\mathrm{CH}_{3}{ }^{\text {Mes }}\right), 21.0\left(p-\mathrm{CH}_{3}{ }^{\text {Mes }}\right),-0.1$ $\left({ }^{1} J_{\mathrm{siC}}=56.2 \mathrm{~Hz}, \mathrm{SiCH}_{3}\right)$.

${ }^{31} \mathrm{P}\left\{{ }^{1} \mathrm{H}\right\}$ NMR $\left(202 \mathrm{MHz}, \mathrm{CD}_{2} \mathrm{Cl}_{2}, 299 \mathrm{~K}\right): \delta=-56.2\left(\mathrm{v}_{1 / 2} \sim 1 \mathrm{~Hz}\right)$.

${ }^{29} \mathrm{Si}\left\{{ }^{1} \mathrm{H}\right\}$ DEPT (99 MHz, $\left.\mathrm{CD}_{2} \mathrm{Cl}_{2}, 299 \mathrm{~K}\right): \delta=-17.4\left(\mathrm{v}_{1 / 2} \sim 2 \mathrm{~Hz}\right)$. 

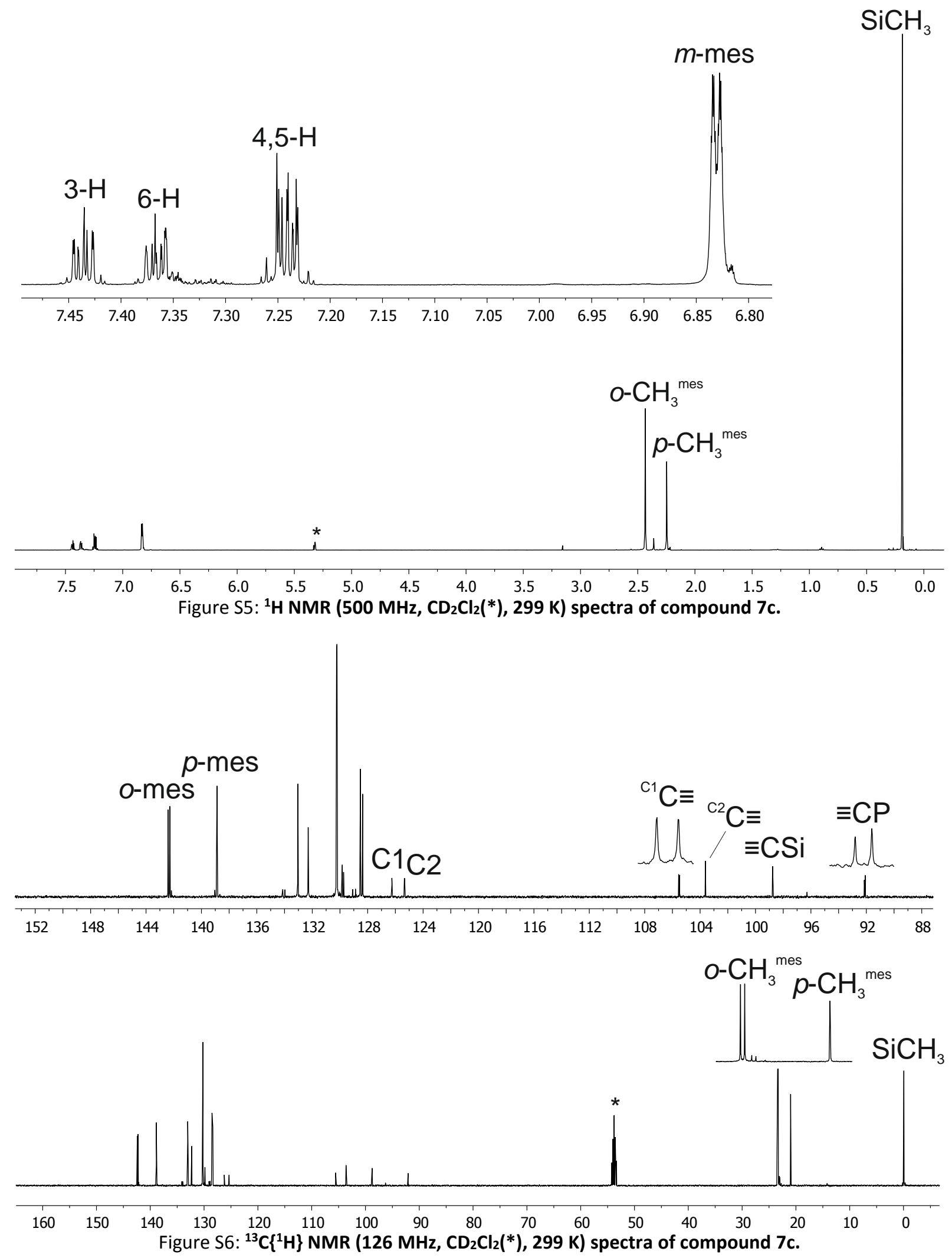


\section{Carboboration Reaction of the Diynes}

\section{Synthesis of compound 8a}<smiles>CC#Cc1ccccc1C(C(=C(c1ccccc1)C(F)(F)F)C(F)(F)F)=C(C(F)(F)F)C(F)(F)F</smiles>

Scheme S4

Compound 7a $(38.3 \mathrm{mg}, 0.1 \mathrm{mmol}, 1 \mathrm{eq})$ and $\mathrm{B}\left(\mathrm{C}_{6} \mathrm{~F}_{5}\right)_{3}(51.2 \mathrm{mg}, 0.1 \mathrm{mmol}$, $1 \mathrm{eq})$ were both dissolved in toluene $(\sim 2 \mathrm{~mL}$ each). The solutions were combined at room temperature and then stirred at $60{ }^{\circ} \mathrm{C}$ overnight. On the next day the solvent was removed in vacuo and $n$-pentane $(\sim 5 \mathrm{~mL})$ was added and directly removed in vacuo again (comment: this procedure was repeated one more time to remove remaining toluene) to give compound $8 \mathrm{a}$

(80 mg, $0.9 \mathrm{mmol}, 89 \%$ ) as a slightly yellow solid. Single crystals suitable for the X-ray crystal structure analysis were obtained from a solution of compound $8 \mathbf{a}$ in benzene at room temperature.

IR (KBr): ṽ $\left[\mathrm{cm}^{-1}\right]=3063(\mathrm{w}), 2962(\mathrm{~m}), 2903(\mathrm{w}), 2154(\mathrm{~m}), 1646(\mathrm{~m}), 1518(\mathrm{~s}), 1464(\mathrm{~s}), 1384(\mathrm{~m})$, $1316(w), 1288(m), 1251(m), 1214(w), 1189(w), 1098(s), 1054(m), 985(s), 924(m), 862(s), 844$ (s), $785(w), 761(m), 744(m), 712(w), 692(m), 643(w), 616(w)$.

Elemental analysis: : Calcd for $\mathrm{C}_{43} \mathrm{H}_{23} \mathrm{BF}_{15} \mathrm{PSi}: \mathrm{C}, 57.74 ; \mathrm{H}, 2.59$. Found: $\mathrm{C}, 57.80, \mathrm{H}, 2.47$.

Melting point (DSC): $210^{\circ} \mathrm{C}$.

${ }^{1} \mathrm{H}$ NMR (500 MHz, C $\left.7 \mathrm{D}_{8}, 299 \mathrm{~K}\right): \delta=7.28(\mathrm{~m}, 4 \mathrm{H}, o-\mathrm{Ph}), 7.23\left(\mathrm{dm},{ }^{3} \mathrm{~J}_{\mathrm{HH}}=7.8 \mathrm{~Hz}, 1 \mathrm{H}, 3-\mathrm{H}\right), 7.09(\mathrm{dm}$, $\left.{ }^{3} \mathrm{~J}_{\mathrm{HH}}=7.9 \mathrm{~Hz}, 1 \mathrm{H}, 6-\mathrm{H}\right), 6.84(\mathrm{~m}, 2 \mathrm{H}, p-\mathrm{Ph}), 6.73(\mathrm{~m}, 4 \mathrm{H}, m-\mathrm{Ph}), 6.68(\mathrm{~m}, 1 \mathrm{H}, 4-\mathrm{H}), 6.60(\mathrm{~m}, 1 \mathrm{H}, 5-\mathrm{H})$, $0.04\left(\mathrm{~s},{ }^{2} \mathrm{~J}_{\mathrm{SiH}}=7.1 \mathrm{~Hz}, \mathrm{SiCH}_{3}\right)$.

${ }^{13} \mathrm{C}\left\{{ }^{1} \mathrm{H}\right\}$ NMR (126 MHz, $\left.\mathrm{C}_{7} \mathrm{D}_{8}, 299 \mathrm{~K}\right): \delta=164.9(\mathrm{br},=\mathrm{CB}), 140.7\left(\mathrm{~d},{ }^{1} \mathrm{~J}_{\mathrm{PC}}=53.0 \mathrm{~Hz},=\mathrm{CP}\right), 136.7(\mathrm{br}, \mathrm{C} 1)$, $136.2(\mathrm{C} 3), 132.4\left(\mathrm{~d},{ }^{4} \mathrm{~J}_{\mathrm{PC}}=2.8 \mathrm{~Hz}, p-\mathrm{Ph}\right), 132.1\left(\mathrm{~d},{ }^{2} \mathrm{~J}_{\mathrm{PC}}=9.3 \mathrm{~Hz}, o-\mathrm{Ph}\right), 129.2\left(\mathrm{~d},{ }^{3} \mathrm{JPC}_{\mathrm{PC}}=10.3 \mathrm{~Hz}, m-\mathrm{Ph}\right.$ ), 129.0 (C4), 128.8 (C5), 127.0 (d, $\left.{ }^{3} J_{\mathrm{PC}}=2.6 \mathrm{~Hz}, \mathrm{C} 6\right), 125.2$ (d, $\left.{ }^{1} J_{\mathrm{PC}}=42.6 \mathrm{~Hz}, i-\mathrm{Ph}\right), 122.4$ (d, ${ }^{3} \mathrm{PC}_{\mathrm{PC}}=6.1 \mathrm{~Hz}$, C2), $103.2\left(d,{ }^{4} J_{\mathrm{PC}}=0.9 \mathrm{~Hz},{ }^{\mathrm{C} 2} \mathrm{C} \equiv\right), 99.7(\equiv \mathrm{CSi}),-0.6\left({ }^{1} J_{\mathrm{SiC}}=57.4 \mathrm{~Hz}, \mathrm{SiCH}_{3}\right),\left[\mathrm{C}_{6} \mathrm{~F}_{5}\right.$ not listed].

${ }^{11} B\left\{{ }^{1} \mathrm{H}\right\}$ NMR (160 MHz, $\left.C_{7} D_{8}, 299 \mathrm{~K}\right): \delta=-5.1\left(\mathrm{v}_{1 / 2} \sim 400 \mathrm{~Hz}\right)$.

${ }^{31} \mathrm{P}\left\{{ }^{1} \mathrm{H}\right\}$ NMR (202 MHz, $\left.\mathrm{C}_{7} \mathrm{D}_{8}, 299 \mathrm{~K}\right): \delta=17.2\left(\mathrm{v}_{1 / 2} \sim 70 \mathrm{~Hz}\right)$.

${ }^{29} \mathrm{Si}\left\{{ }^{1} \mathrm{H}\right\}$ DEPT NMR (99 MHz, $\left.\mathrm{C}_{7} \mathrm{D}_{8}, 299 \mathrm{~K}\right):-17.2\left(\mathrm{v}_{1 / 2} \sim 2 \mathrm{~Hz}\right)$.

${ }^{19} \mathrm{~F}$ NMR $\left(470 \mathrm{MHz}, \mathrm{C}_{7} \mathrm{D}_{8}, 299 \mathrm{~K}\right): \delta=-128.8(\mathrm{br}, 4 \mathrm{~F}, o),-156.7(\mathrm{br}, 2 \mathrm{~F}, p),-163.4(\mathrm{~m}, 4 \mathrm{~F}, m)\left(\mathrm{BC}_{6} \mathrm{~F}_{5}\right)$ $\left.\left[\Delta \delta^{19} \mathrm{~F}_{m, p}=6.7\right],-136.8(\mathrm{br}, 2 \mathrm{~F}, o),-154.5\left(\mathrm{t},{ }^{3}\right]_{\mathrm{FF}}=21.3 \mathrm{~Hz}, 1 \mathrm{~F}, p\right),-162.2(\mathrm{~m}, 2 \mathrm{~F}, m)\left(\mathrm{C}_{6} \mathrm{~F}_{5}\right)\left[\Delta \delta^{19} \mathrm{~F}_{m, p}=\right.$ 7.7].

${ }^{1} \mathrm{H}^{13}{ }^{13} \mathrm{C}$ GHSQC (500 MHz / $126 \mathrm{MHz}, \mathrm{C}_{7} \mathrm{D}_{8}, 299 \mathrm{~K}$ ): $\delta^{1} \mathrm{H} / \delta^{13} \mathrm{C}=7.28 / 132.1$ (o-Ph / o-Ph), 7.23 / 136.2 (3-H / C3), 7.09 / 127.0 (6-H / C6), 6.84 / 132.4 (p-Ph / p-Ph), 6.73 / 129.2 (m-Ph / m-Ph), 6.68 / 129.0 (4-H / C4), 6.60 / 128.8 (5-H / C5), 0.04 / -0.6 ( $\left.\mathrm{SiCH}_{3} / \mathrm{SiCH}_{3}\right)$.

${ }^{1} \mathrm{H}^{13} \mathrm{C}$ GHMBC (500 MHz / 126MHz, C7 $\mathrm{D}_{8}, 299 \mathrm{~K}$ ) [selected resonances]: $\delta^{1} \mathrm{H} / \delta^{13} \mathrm{C}=7.28 / 132.4$, 131.1 (o-Ph / p-Ph, o-Ph), 7.23 / 136.7, 128.8, 103.2 (3-H / C1, C5, $\left.{ }^{C 2} \mathrm{C} \equiv\right)$, 7.09 / 140.7, 129.0, 122.4 
(6-H / =CP, C4, C2), 6.73 / 132.1, 129.2, 125.2 (m-Ph / o-Ph, m-Ph, i-Ph), 6.68 / 127.0, 122.4 (4-H / C6, C2), 6.60 / 136.7, 136.2 (5-H / C1, C3), 0.04 / 99.7, -0.6 ( $\left.\mathrm{SiCH}_{3} / \equiv \mathrm{CSi}, \mathrm{SiCH}_{3}\right)$.
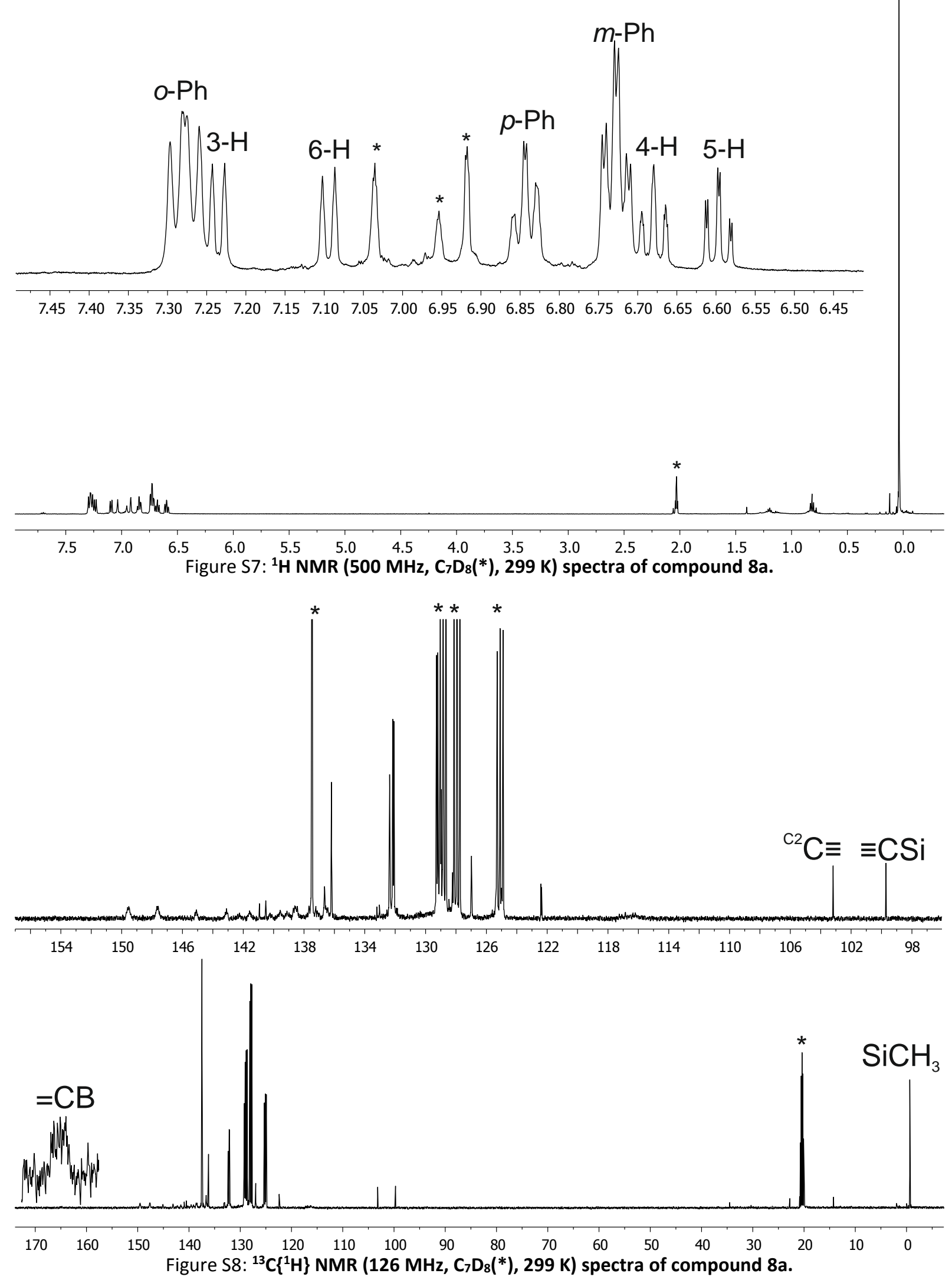


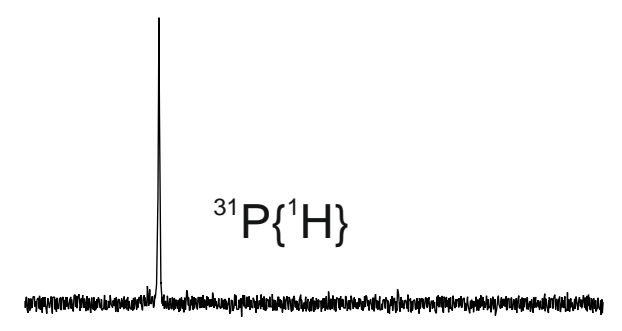

$\begin{array}{llllllllllll}40 & 30 & 20 & 10 & 0 & -10 & -20 & -30 & -40 & -50 & -60 & -70\end{array}$
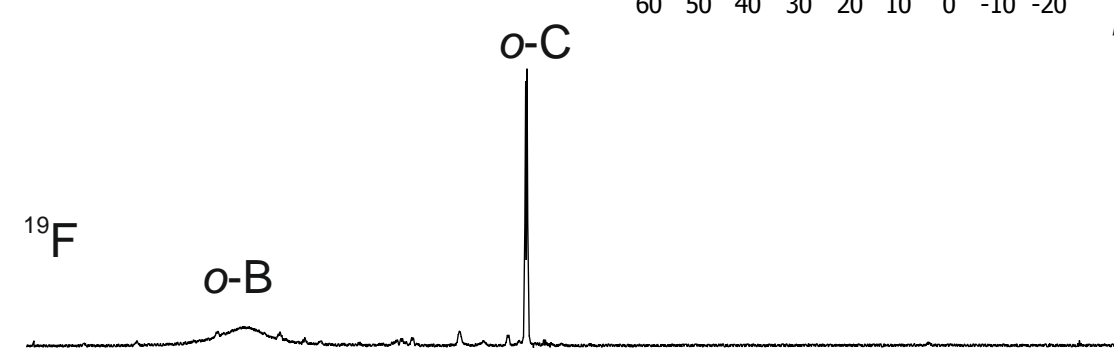

$-135$

$-140$

Figure S9: ${ }^{19} \mathrm{~F}$ NMR $\left(470 \mathrm{MHz}, \mathrm{C}_{7} \mathrm{D}_{8}, 299 \mathrm{~K}\right),{ }^{11} \mathrm{~B}\left\{{ }^{1} \mathrm{H}\right\}$ NMR $(160 \mathrm{MHz}),{ }^{31} \mathrm{P}\left\{{ }^{1} \mathrm{H}\right\} \mathrm{NMR}(202 \mathrm{MHz})$ and
${ }^{29} \mathrm{Si}\left\{{ }^{1} \mathrm{H}\right\}$ DEPT

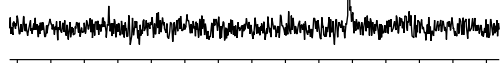

$\begin{array}{lllllll}5 & 0 & -5 & -10 & -15 & -20 & -25\end{array}$

$m-\mathrm{B}$

${ }^{29} \mathrm{Si}\left\{{ }^{1} \mathrm{H}\right\}$ DEPT NMR (99 MHz) spectra of compound 8a.

Figure S10: X-ray crystal structure analysis of compound 8a: formula $\mathrm{C}_{43} \mathrm{H}_{23} \mathrm{BF}_{15} \mathrm{PSi}, \mathrm{M}=894.48$, pale yellow crystal, $0.12 \times 0.07 \times 0.04 \mathrm{~mm}, a=10.4133(4), b=11.9484(5), c=18.3165(9) \AA, \alpha=$ 104.187(2), $b=95.648(1), \gamma=95.187(2)^{\circ}, V=2183.3(2) \AA^{3}, \rho_{\text {calc }}=1.361 \mathrm{gcm}^{-3}, \mu=0.184 \mathrm{~mm}^{-1}$, empirical absorption correction $(0.978 \leq T \leq 0.992), Z=2$, triclinic, space group $P \overline{1}$ (No. 2$), \lambda=$ $0.71073 \AA, T=223(2) \mathrm{K}, \omega$ and $\phi$ scans, 20885 reflections collected $( \pm h, \pm k, \pm /),[(\sin \theta) / \lambda]=0.59 \AA^{-1}$, 7473 independent $\left(R_{\text {int }}=0.049\right)$ and 5903 observed reflections $[/>2 \sigma(I)], 587$ refined parameters, $R=$ $0.073, w R^{2}=0.195$, max. (min.) residual electron density $0.45(-0.32)$ e. $\AA^{-3}$, hydrogen atoms were calculated and refined as riding atoms. 


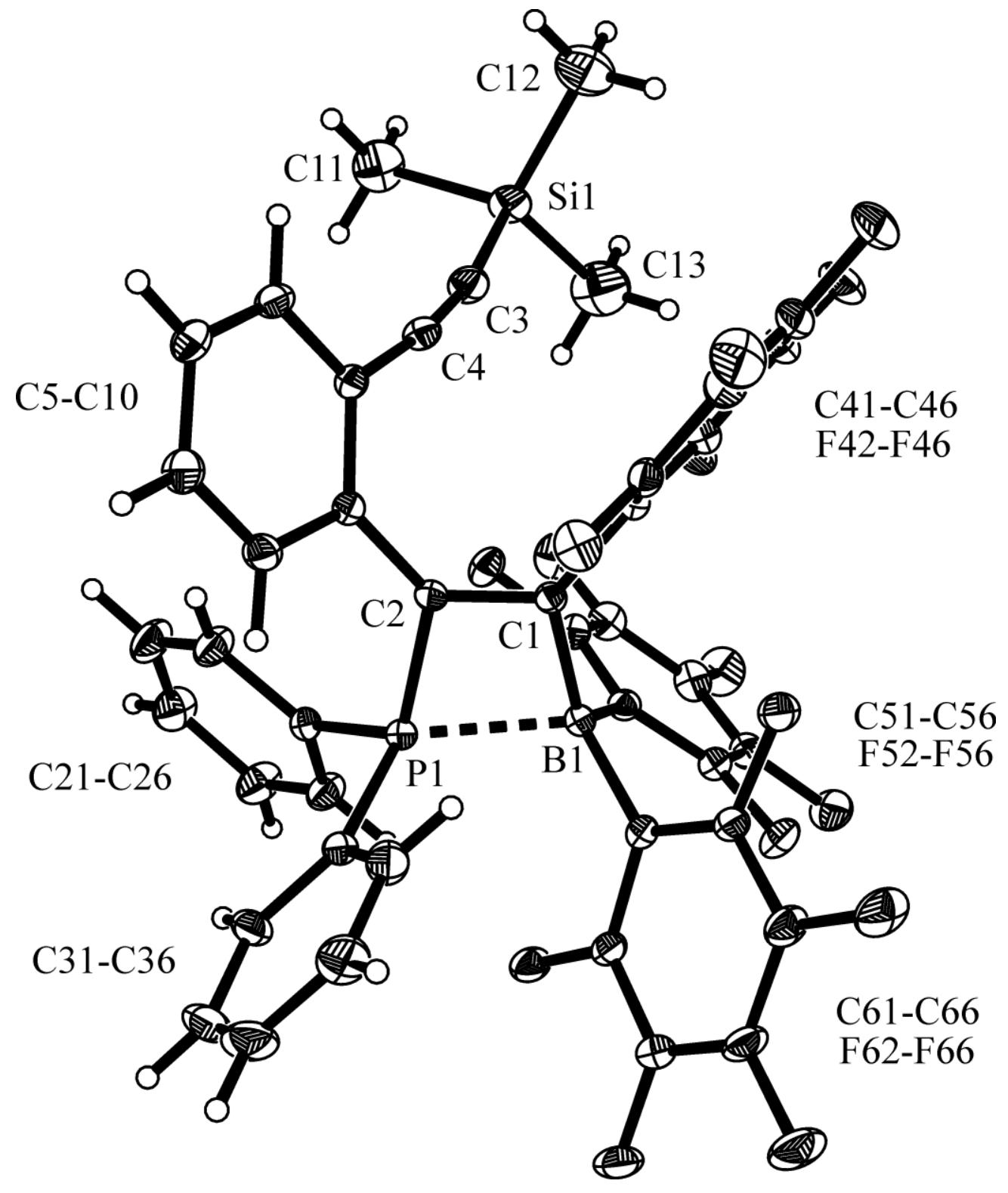

Synthesis of compound $8 b$

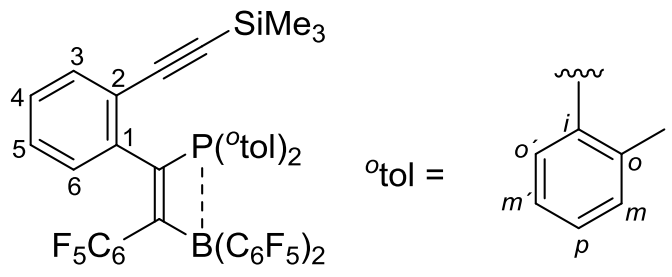

Scheme S5

Compound $7 \mathrm{~b}$ ( $82.2 \mathrm{mg}, 0.2 \mathrm{mmol}, 1 \mathrm{eq})$ was dissolved in toluene $(\sim 2 \mathrm{~mL})$ and a solution of $B\left(C_{6} F_{5}\right)_{3}(102 \mathrm{mg}$, $0.2 \mathrm{mmol}, 1 \mathrm{eq})$ in toluene $(\sim 2 \mathrm{~mL})$ was added at room temperature. The resulting solution was stirred at $60^{\circ} \mathrm{C}$ overnight, then cooled to room temperature and then all volatiles were removed in vacuo. Subsequently $n$-pentane $(\sim 10 \mathrm{~mL})$ was added and directly removed in vacuo to remove remaining toluene. The residue was redissolved in $n$-pentane $(\sim 10 \mathrm{~mL})$ and stored at $-40{ }^{\circ} \mathrm{C}$ for several hours. The formed slightly yellow solid was collected and redissolved in $n$-pentane $(\sim 5 \mathrm{~mL})$ again and crystallized at 
$-40{ }^{\circ} \mathrm{C}$. The obtained colorless solid was dried in vacuo to give compound $8 \mathbf{b}$ (95 mg, $0.13 \mathrm{mmol}$, $67 \%)$.

IR (KBr): $\tilde{v}\left[\mathrm{~cm}^{-1}\right]=3063(w), 2961(w), 2156(w), 1645(\mathrm{~m}), 1519(\mathrm{~s}), 1466(\mathrm{~s}), 1385(\mathrm{w}), 1313(\mathrm{w})$, $1286(\mathrm{~m}), 1250(\mathrm{~m}), 1209(\mathrm{w}), 1107(\mathrm{~s}), 1054(\mathrm{w}), 984(\mathrm{~s}), 918(\mathrm{~m}), 859(\mathrm{~s}), 781(\mathrm{w}), 756(\mathrm{~m}), 714(\mathrm{w})$, $644(w), 619(w)$.

Elemental analysis: Calcd for $\mathrm{C}_{45} \mathrm{H}_{27} \mathrm{BF}_{15} \mathrm{PSi}: \mathrm{C}, 58.58 ; \mathrm{H}, 2.95$. Found: $\mathrm{C}, 58.88 ; \mathrm{H}, 2.94$.

Melting point (DSC): $181^{\circ} \mathrm{C}$.

${ }^{1} \mathrm{H}$ NMR (500 MHz, $\left.\mathrm{CD}_{2} \mathrm{Cl}_{2}, 299 \mathrm{~K}\right): \delta=7.47(\mathrm{~m}, 1 \mathrm{H}, 3-\mathrm{H}), 7.45\left(\mathrm{~m}, 2 \mathrm{H}, p^{-0}\right.$ tol), $7.36\left(\mathrm{br}, 2 \mathrm{H}, o^{\prime}-{ }^{\circ}\right.$ tol), $7.27(\mathrm{~m}, 1 \mathrm{H}, 4-\mathrm{H}), 7.24$ (br m, $2 \mathrm{H}, m^{-}{ }^{\circ}$ tol $), 7.18\left(\mathrm{~m}, 2 \mathrm{H}, m^{\prime}{ }^{-}{ }^{\circ}\right.$ tol $), 7.16(\mathrm{~m}, 1 \mathrm{H}, 5-\mathrm{H}), 6.98(\mathrm{~m}, 1 \mathrm{H}, 6-\mathrm{H})$, $2.05\left(\mathrm{~s}, 6 \mathrm{H}, \mathrm{o}-\mathrm{CH}_{3}{ }^{\mathrm{tol}}\right), 0.09\left(\mathrm{~s},{ }^{2} \mathrm{~J}_{\mathrm{SiH}}=7.1 \mathrm{~Hz}, 9 \mathrm{H}, \mathrm{SiCH}_{3}\right)$.

${ }^{13} \mathrm{C}\left\{{ }^{1} \mathrm{H}\right\}$ NMR (126 MHz, CD $\left.\mathrm{Cl}_{2}, 299 \mathrm{~K}\right): \delta=164.5$ (br, BC=), 142.9 (d, ${ }^{2} J_{\mathrm{PC}}=9.7 \mathrm{~Hz}, \mathrm{o}^{-}{ }^{\circ}$ tol), $141.5\left(\mathrm{~d},{ }^{1} J_{\mathrm{PC}}\right.$ $=52.0 \mathrm{~Hz}, \mathrm{PC}=), 137.4(\mathrm{C} 1), 136.2(\mathrm{C} 3), 134.6\left(\mathrm{~d},{ }^{2} J_{\mathrm{PC}}=8.7 \mathrm{~Hz}, o^{\prime}-^{\circ}\right.$ tol), 132.7 (d, ${ }^{4} J_{\mathrm{PC}}=2.8 \mathrm{~Hz}, p-{ }^{\circ}$ tol), $132.2\left(\mathrm{br} \mathrm{d},{ }^{3} \mathrm{JPC}_{\mathrm{PC}}=8.7 \mathrm{~Hz}, \mathrm{~m}^{\circ}{ }^{\circ} \mathrm{tol}\right), 129.1$ (C5), 128.9 (C4), $127.6\left(\mathrm{br} \mathrm{d},{ }^{3} \mathrm{~J}_{\mathrm{PC}}=2.3 \mathrm{~Hz}, \mathrm{C} 6\right), 126.9\left(\mathrm{~d},{ }^{3} \mathrm{JPC}_{\mathrm{PC}}=\right.$ $9.7 \mathrm{~Hz}, m^{\prime}{ }^{\circ}{ }^{\circ}$ tol), $125.2\left(\mathrm{~d},{ }^{1} \mathrm{~J}_{\mathrm{PC}}=38,7 \mathrm{~Hz}, i-{ }^{\circ}\right.$ tol) $, 122.3\left(\mathrm{~d},{ }^{3} \mathrm{~J}_{\mathrm{PC}}=5.3 \mathrm{~Hz}, \mathrm{C} 2\right), 102.9$ (d, ${ }^{4} J_{\mathrm{PC}}=1.0 \mathrm{~Hz}$, $\left.{ }^{\mathrm{C} 2} \mathrm{C} \equiv\right), 99.8(\equiv \mathrm{CSi}), 21.8\left(\mathrm{~d},{ }^{3} \mathrm{JPC}_{\mathrm{PC}}=4.6 \mathrm{~Hz}, 0-\mathrm{CH}_{3}{ }^{\mathrm{tol}}\right),-0.6\left({ }^{1} J_{\mathrm{SiC}}=56.5 \mathrm{~Hz}, \mathrm{SiCH}_{3}\right),\left[\mathrm{C}_{6} \mathrm{~F}_{5}\right.$ not listed].

${ }^{29} \mathrm{Si}\left\{{ }^{1} \mathrm{H}\right\}$ DEPT (99 MHz, $\left.\mathrm{CD}_{2} \mathrm{Cl}_{2}, 299 \mathrm{~K}\right): \delta=-17.2\left(\mathrm{v}_{1 / 2} \sim 2 \mathrm{~Hz}\right)$.

${ }^{31} \mathrm{P}\left\{{ }^{1} \mathrm{H}\right\}$ NMR (202 MHz, $\left.\mathrm{CD}_{2} \mathrm{Cl}_{2}, 299 \mathrm{~K}\right): \delta=20.1\left(\mathrm{v}_{1 / 2} \sim 80 \mathrm{~Hz}\right)$.

${ }^{10} \mathrm{~B}\left\{{ }^{1} \mathrm{H}\right\}$ NMR (54 MHz, $\left.\mathrm{CD}_{2} \mathrm{Cl}_{2}, 299 \mathrm{~K}\right): \delta=-3.6\left(\mathrm{v}_{1 / 2} \sim 150 \mathrm{~Hz}\right)$.

${ }^{19} \mathrm{~F}$ NMR $\left(470 \mathrm{MHz}, \mathrm{CD}_{2} \mathrm{Cl}_{2}, 299 \mathrm{~K}\right): \delta=-127.2(\mathrm{br}, 4 \mathrm{~F}, o),-157.3\left(\mathrm{t}, 2 \mathrm{~F}, 3_{\mathrm{FF}}=19.2 \mathrm{~Hz}, p\right),-164.5(\mathrm{br}$, $4 \mathrm{~F}, m)\left(\mathrm{BC}_{6} \mathrm{~F}_{5}\right)\left[\Delta \delta^{19} \mathrm{~F}_{m, p}=7.2\right],-135.9(\mathrm{br}, 2 \mathrm{~F}, o),-155.9\left(\mathrm{t}, 1 \mathrm{~F},{ }^{3} \mathrm{~J}_{\mathrm{FF}}=21.2 \mathrm{~Hz}, p\right),-163.8(\mathrm{~m}, 2 \mathrm{~F}$, $m)\left(\mathrm{C}_{6} \mathrm{~F}_{5}\right)\left[\Delta \delta^{19} \mathrm{~F}_{m, p}=7.9\right]$.

${ }^{1} \mathrm{H},{ }^{13} \mathrm{C}$ GHSQC (500 MHz / $\left.126 \mathrm{MHz}, \mathrm{CD}_{2} \mathrm{Cl}_{2}, 299 \mathrm{~K}\right): \delta^{1} \mathrm{H} / \delta^{13} \mathrm{C}=7.47 / 136.2$ (3-H / C3), 7.45 / 132.7

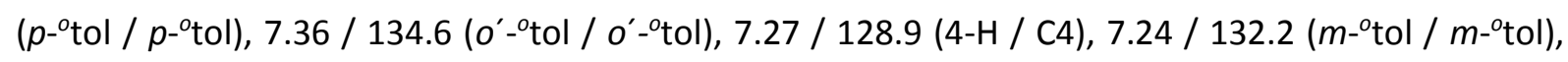
7.18 / 126.9 ( $m^{\prime}-{ }^{\circ}$ tol / $m^{\prime}-{ }^{\circ}$ tol), $7.16 / 129.1$ (5-H / C5), 6.98 / 127.6 (6-H / C6), $2.05 / 21.8$ (o- $-\mathrm{CH}_{3}{ }^{\text {tol }} /$ $\left.0-\mathrm{CH}_{3}{ }^{\mathrm{tol}}\right), 0.09 /-0.6\left(\mathrm{SiCH}_{3} / \mathrm{SiCH}_{3}\right)$.

${ }^{1} \mathrm{H},{ }^{13} \mathrm{C}$ GHMBC (500 MHz / $126 \mathrm{MHz}, \mathrm{CD}_{2} \mathrm{Cl}_{2}, 299 \mathrm{~K}$ ) [selected traces]: $\delta^{1} \mathrm{H} / \delta^{13} \mathrm{C}=7.47$ / 137.4, 129.1, 102.9 (3-H / C1, C5, $\left.{ }^{\mathrm{C} 2} \mathrm{C} \equiv\right), 7.45 / 142.9,134.6$ ( $p-^{\circ}{ }^{\circ}$ tol / o- ${ }^{\circ}$ tol, $o^{\prime}-{ }^{\circ}$ tol), 7.27 / 127.6, 122.3 (3-H / C6, C2), $7.24 / 126.9,125.2,21.8\left(m^{-}{ }^{\circ}\right.$ tol $/ m^{\prime}-{ }^{o}$ tol, $i-{ }^{\circ}$ tol, $\left.o-C_{3}{ }^{\text {tol }}\right), 7.18 / 134.6,132.2,125.2\left(m^{\prime}-{ }^{o}\right.$ tol / $o^{\prime}-{ }^{\circ}$ tol, $m-^{\circ}{ }^{\circ}$ tol, $i-{ }^{\circ}$ tol), $7.16 / 137.4,136.2(5-\mathrm{H} / \mathrm{C} 1, \mathrm{C} 3), 6.98 / 141.5,128.9,122.3(6-\mathrm{H} / \mathrm{PC}=, \mathrm{C} 4, \mathrm{C2})$, $2.05 / 142.9,132.2,125.2\left(o-\mathrm{CH}_{3}{ }^{\text {tol }} / o^{-}{ }^{o}\right.$ tol, $m-{ }^{\circ}$ tol, $i-{ }^{\circ}$ tol $), 0.09 / 99.8,-0.6\left(\mathrm{SiCH}_{3} / \equiv \mathrm{CSi}, \mathrm{SiCH}_{3}\right)$. 


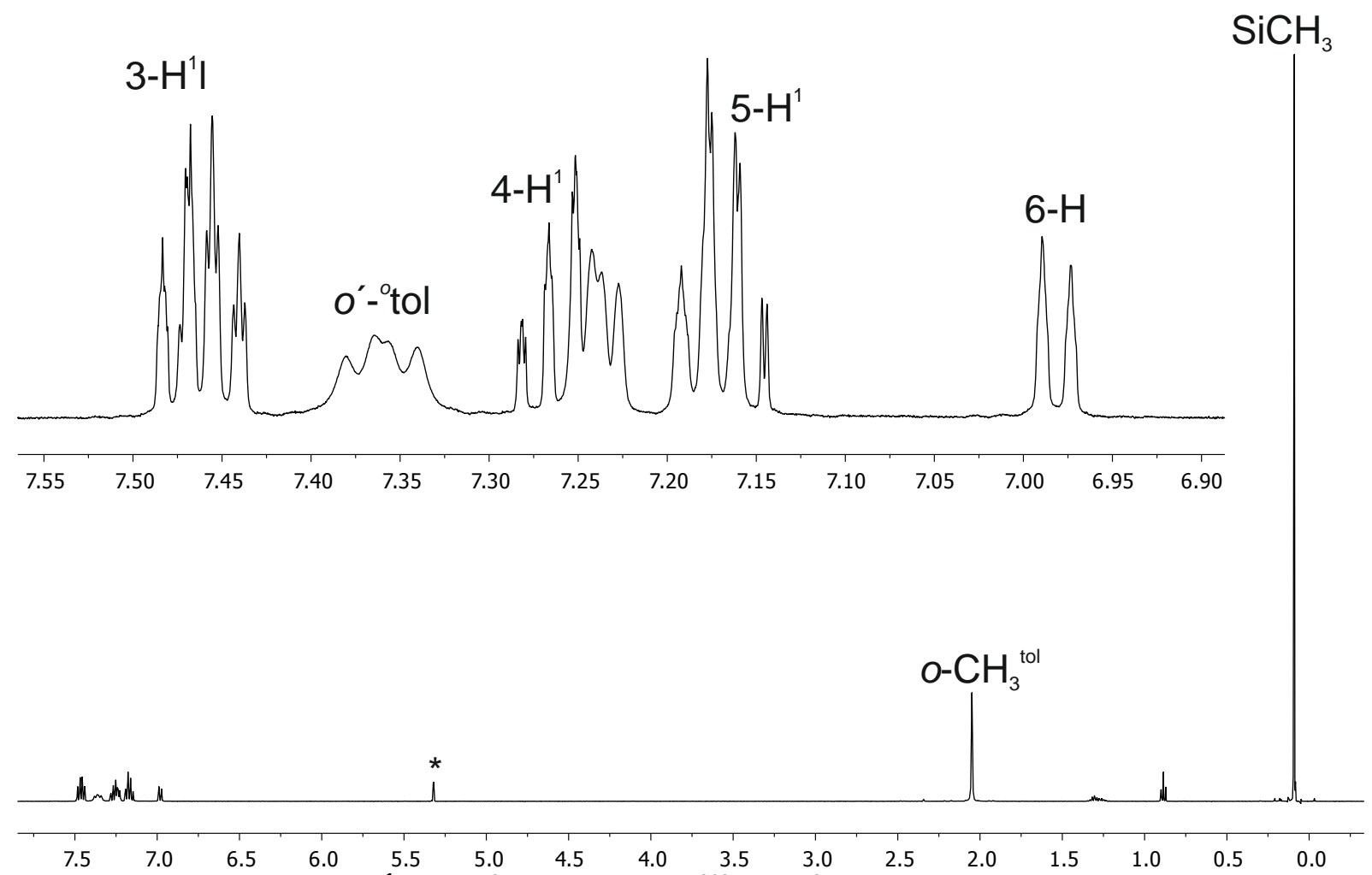

Figure S11: ${ }^{1} \mathrm{H}$ NMR (500 MHz, $\mathrm{CD}_{2} \mathrm{Cl}_{2}\left({ }^{*}\right)$, $299 \mathrm{~K}$ ) spectra of compound $8 \mathrm{~b}$.
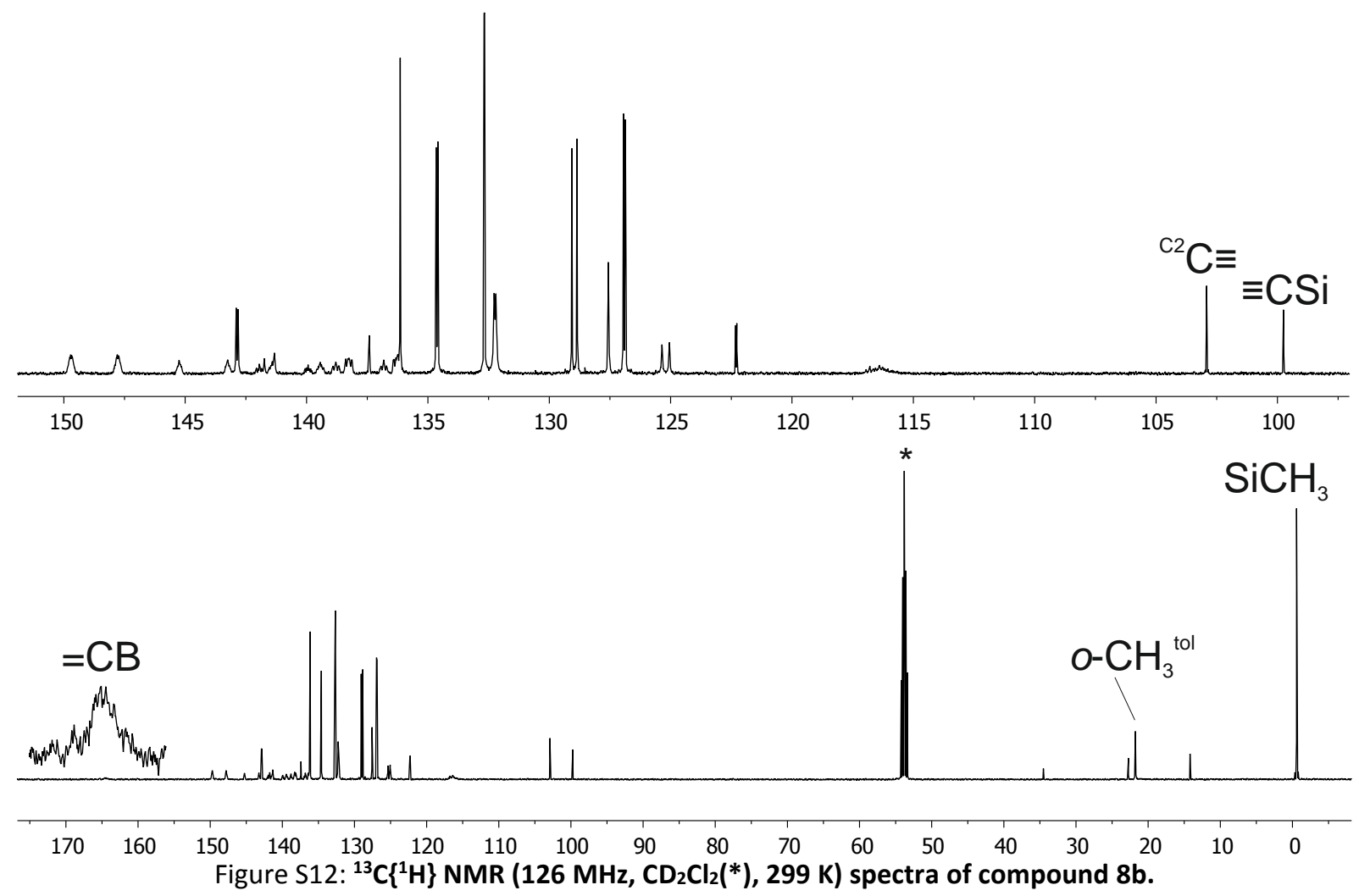

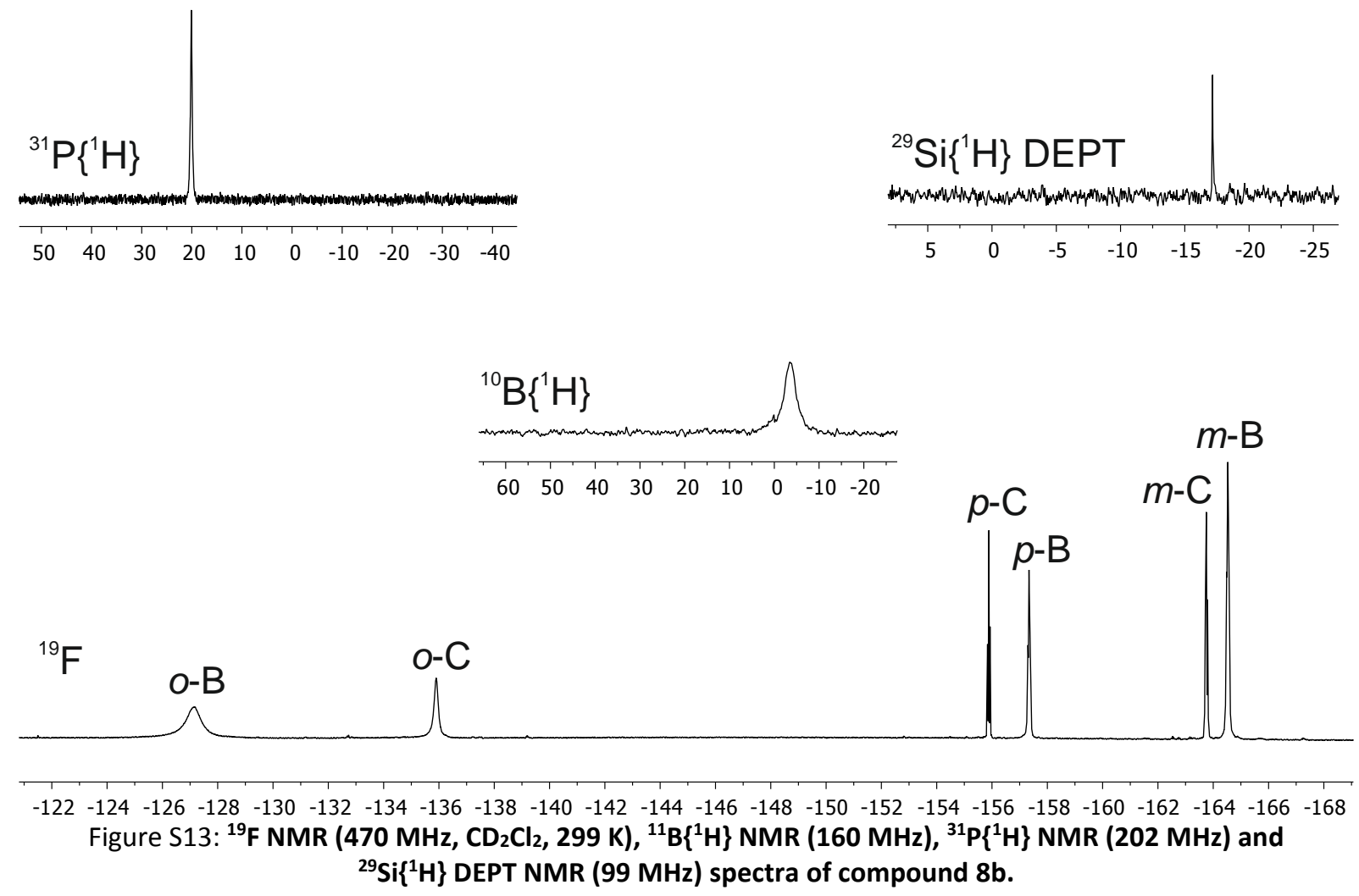

\section{Synthesis of compound 9}

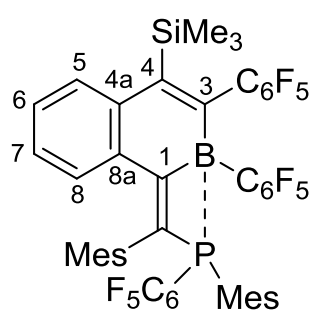

Scheme S6

Compound 7c (325 mg, $0.7 \mathrm{mmol}, 1 \mathrm{eq})$ and $\mathrm{B}\left(\mathrm{C}_{6} \mathrm{~F}_{5}\right)_{3}(356 \mathrm{mg}, 0.7 \mathrm{mmol}, 1 \mathrm{eq})$ were dissolved separately in toluene (each $\sim 3 \mathrm{~mL}$ ). The solutions were combined at room temperature and then stirred for $3 \mathrm{~h}$ at $80^{\circ} \mathrm{C}$. The resulting deep red solution was cooled to room temperature and the solvent was removed in vacuo. Then $n$-pentane $(\sim 5 \mathrm{~mL})$ was added and directly removed in vacuo (to remove remaining toluene). The residue was redissolved in $n$-pentane $(\sim 8 \mathrm{~mL})$ and stored at $-40{ }^{\circ} \mathrm{C}$ overnight to give a suspension. The supernatant was removed using a pipette and the colorless residue dried in vacuo to give compound 9 (564 mg, $0.58 \mathrm{mmol}, 82 \%$ ) as a colorless solid. Single crystals suitable for the X-ray diffraction were obtained by storing a diluted solution of compound 9 in $n$-heptane for several days at $-40{ }^{\circ} \mathrm{C}$.

IR (KBr): $\tilde{\mathbf{v}}\left[\mathrm{cm}^{-1}\right]=3649(\mathrm{w}), 2958(\mathrm{~m}), 2925(\mathrm{~m}), 2860(\mathrm{w}), 1645(\mathrm{~m}), 1608(\mathrm{~m}), 1518(\mathrm{~s}), 1477(\mathrm{~s})$, $1380(\mathrm{~m}), 1294(\mathrm{~m}), 1252(\mathrm{~m}), 1146(\mathrm{w}), 1093(\mathrm{~s}), 1031(\mathrm{~m}), 984(\mathrm{~s}), 913(\mathrm{~m}), 847(\mathrm{~s}), 782(\mathrm{w}), 769$ $(w), 759(w), 697(w), 639(w), 557(w), 506(w)$.

Elemental analysis: Calcd for $\mathrm{C}_{49} \mathrm{H}_{35} \mathrm{BF}_{15} \mathrm{PSi}$ : $\mathrm{C}, 60.14 ; \mathrm{H}, 3.60$. Found: $\mathrm{C}, 60.36 ; \mathrm{H}, 3.56$.

Melting point (DSC): $109^{\circ} \mathrm{C}$. 
${ }^{1} \mathrm{H}$ NMR $\left(600 \mathrm{MHz}, \mathrm{C}_{6} \mathrm{D}_{6}, 299 \mathrm{~K}\right): \delta=7.52\left(\mathrm{~d},{ }^{3} \mathrm{H}_{\mathrm{HH}}=7.9 \mathrm{~Hz}, 1 \mathrm{H}, 5-\mathrm{H}\right), 6.94\left(\mathrm{~d},{ }^{3} \mathrm{JHH}_{\mathrm{HH}}=7.6 \mathrm{~Hz}, 1 \mathrm{H}, 8-\mathrm{H}\right)$, $6.90(\mathrm{~m}, 1 \mathrm{H}, 6-\mathrm{H}), 6.71(\mathrm{~m}, 1 \mathrm{H}, 7-\mathrm{H}), 6.66\left(\mathrm{br}, 2 \mathrm{H}, m^{-}{ }^{\mathrm{C}} \mathrm{Mes}\right), 6.49\left(\mathrm{~d},{ }^{4} J_{\mathrm{PH}}=3.3 \mathrm{~Hz}, 2 \mathrm{H}, m^{\mathrm{P}}{ }^{\mathrm{Mes}}\right), 2.06$ (s, $\left.3 \mathrm{H}, p^{-}{ }^{\mathrm{C}} \mathrm{CH}_{3}{ }^{\text {Mes }}\right), 2.03\left(\mathrm{br}, 6 \mathrm{H}, o_{-}{ }^{\mathrm{C}} \mathrm{CH}_{3}{ }^{\text {Mes }}\right), 2.00\left(\mathrm{~s}, 6 \mathrm{H}, o_{-}{ }^{\mathrm{P}} \mathrm{CH}_{3}{ }^{\text {Mes }}\right), 1.93\left(\mathrm{~s}, 3 \mathrm{H}, p-{ }^{\mathrm{P}} \mathrm{CH}_{3}{ }^{\text {Mes }}\right),-0.02\left(\mathrm{~s}^{2}{ }^{2} \mathrm{~J}_{\mathrm{siH}}=\right.$ $\left.6.4 \mathrm{~Hz}, \mathrm{SiCH}_{3}\right)$.

${ }^{13} \mathrm{C}\left\{{ }^{1} \mathrm{H}\right\}$ NMR (151 MHz, $\left.\mathrm{C}_{6} \mathrm{D}_{6}, 299 \mathrm{~K}\right): \delta=183.5(\mathrm{br} \mathrm{d}, \mathrm{J} \sim 50 \mathrm{~Hz}, \mathrm{C} 1), 156.9(\mathrm{br}, \mathrm{C} 4), 145.7(=\mathrm{CP})$ t, 142.4 (d, $\left.{ }^{2} J_{\mathrm{PC}}=11.1 \mathrm{~Hz}, \mathrm{o}^{\mathrm{P}} \mathrm{Mes}\right), 142.2\left(\mathrm{~d},{ }^{4} \mathrm{JPC}_{\mathrm{PC}}=2.2 \mathrm{~Hz}, \mathrm{p}^{\mathrm{P}} \mathrm{Mes}\right), 138.4\left(\mathrm{~d},{ }^{4} \mathrm{~J}_{\mathrm{PC}}=2.6 \mathrm{~Hz}, \mathrm{C} 4 \mathrm{a}\right), 138.2(\mathrm{br}$,

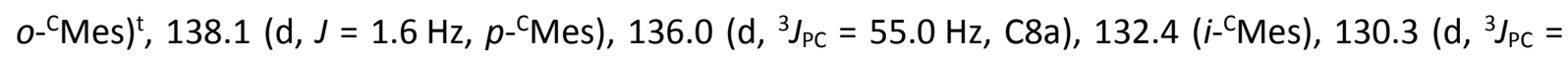
$7.5 \mathrm{~Hz}, m^{\mathrm{P}}{ }^{\mathrm{M}} \mathrm{Mes}$ ), 129.8 (d, $J=0.9 \mathrm{~Hz}, \mathrm{C} 5$ ), 129.3 ( $\mathrm{m}^{\mathrm{C}}{ }^{\mathrm{C} M e s}$ ), 128.5 (C6), 127.4 (d, J = 2.3 Hz, C7), 127.1 (br d, $\left.{ }^{1} J_{\mathrm{PC}}=11.4 \mathrm{~Hz}, i^{\mathrm{P}}{ }^{\mathrm{M}} \mathrm{Mes}\right), 125.6(\mathrm{C} 8), 22.5\left(\mathrm{br} \mathrm{d},{ }^{3} J_{\mathrm{PC}}=9.8 \mathrm{~Hz}, o_{-}{ }^{\mathrm{P}} \mathrm{CH}_{3}{ }^{\text {Mes }}\right), 21.0\left(p^{-}{ }^{\mathrm{C}} \mathrm{CH}_{3}{ }^{\text {Mes }}\right), 20.7$ (d, $\left.{ }^{5} J_{\mathrm{PC}}=1.1 \mathrm{~Hz}, p-{ }^{\mathrm{P}} \mathrm{CH}_{3}{ }^{\mathrm{Mes}}\right), 20.6\left(\mathrm{br}, \mathrm{o}^{-}{ }^{\mathrm{C}} \mathrm{CH}_{3}{ }^{\mathrm{Mes}}\right), 1.9\left({ }^{1} \mathrm{~J}_{\mathrm{SiC}}=52.5 \mathrm{~Hz}, \mathrm{SiCH}_{3}\right)$, n.o. (C3), [C ${ }_{6} \mathrm{~F}_{5}$ not listed].

${ }^{11} B\left\{{ }^{1} H\right\}$ NMR (192 MHz, $\left.C_{7} D_{8}, 299 \mathrm{~K}\right): \delta=15.9\left(v_{1 / 2} \sim 1500 \mathrm{~Hz}\right)$.

${ }^{31} \mathrm{P}\left\{{ }^{1} \mathrm{H}\right\}$ NMR (243 MHz, $\left.\mathrm{C}_{6} \mathrm{D}_{6}, 299 \mathrm{~K}\right): \delta=11.5\left(\mathrm{v}_{1 / 2} \sim 50 \mathrm{~Hz}\right)$.

${ }^{29} \mathrm{Si}\left\{{ }^{1} \mathrm{H}\right\}$ DEPT (119 MHz, $\left.\mathrm{C}_{6} \mathrm{D}_{6}, 299 \mathrm{~K}\right): \delta=-4.2\left(\mathrm{~V}_{1 / 2} \sim 2 \mathrm{~Hz}\right)$.

${ }^{19} \mathrm{~F}$ NMR (564 MHz, $\left.\mathrm{C}_{6} \mathrm{D}_{6}, 299 \mathrm{~K}\right): \delta=-125.8\left(\mathrm{br} \mathrm{m}, 2 \mathrm{~F}, o\right.$ ), $-148.2(\mathrm{br}, 1 \mathrm{~F}, p),-160.0(\mathrm{br}, 2 \mathrm{~F}, m)\left(\mathrm{C}_{6} \mathrm{~F}_{5}\right)$, $\left.\left[\Delta \delta^{19} \mathrm{~F}_{m, p}=11.8\right],-126.4(\mathrm{br}, 2 \mathrm{~F}, o),-154.9\left(\mathrm{t},{ }^{3}\right)_{\mathrm{FF}}=20.0 \mathrm{~Hz}, 1 \mathrm{~F}, p\right),-163.0(\mathrm{~m}, 2 \mathrm{~F}, m)\left(\mathrm{C}_{6} \mathrm{~F}_{5}\right),\left[\Delta \delta^{19} \mathrm{~F}_{m, p}=\right.$ 8.1], $-136.0,-137.9$ (each br, each $1 \mathrm{~F}, o),-158.0\left(\mathrm{t},{ }^{3} \mathrm{FFF}_{\mathrm{FF}}=21.0 \mathrm{~Hz}, 1 \mathrm{~F}, p\right),-163.6(\mathrm{br}, 2 \mathrm{~F}, m)\left(\mathrm{C}_{6} \mathrm{~F}_{5}\right)$, $\left[\Delta \delta^{19} \mathrm{~F}_{m, p}=5.6\right]$.

${ }^{1} H\left\{{ }^{1} H\right\}$ TOCSY (600 MHz, $C_{6} D_{6}, 299 \mathrm{~K}$ ) [selected experiments]: $\delta^{1} H_{\text {irr }} / \delta^{1} H_{\text {res }}=7.52 / 6.94,6.90,6.71$ (5-H / 8-H, 6-H, 7-H).

${ }^{1} \mathrm{H}\left\{{ }^{1} \mathrm{H}\right\}$ NOE (600 MHz, $\left.\mathrm{C}_{6} \mathrm{D}_{6}, 299 \mathrm{~K}\right)$ [selected experiments]: $\delta^{1} \mathrm{H}_{\text {irr }} / \delta^{1} \mathrm{H}_{\text {res }}=7.52 / 6.90,-0.02(5-\mathrm{H} /$ 6-H, $\left.\mathrm{SiCH}_{3}\right), 6.49$ / 2.00, 1.93 ( $\left.m-{ }^{\mathrm{P}} \mathrm{Mes} / \mathrm{O}^{-}{ }^{\mathrm{P}} \mathrm{CH}_{3}{ }^{\mathrm{Mes}}, p^{\mathrm{P}} \mathrm{CH}_{3}{ }^{\mathrm{Mes}}\right),-0.02, / 7.52\left(\mathrm{SiCH}_{3} / 5-\mathrm{H}\right)$.

${ }^{1} \mathrm{H},{ }^{1} \mathrm{H}$ GCOSY (600 MHz / $600 \mathrm{MHz}, \mathrm{C}_{6} \mathrm{D}_{6}, 299 \mathrm{~K}$ ) [selected traces]: $\delta^{1} \mathrm{H} / \delta^{1} \mathrm{H}=7.52 / 6.90(5-\mathrm{H} / 6-\mathrm{H})$, 6.94 / 6.71 (8-H / 7-H), 6.90 / 7.52, 6.71 (6-H / 5-H, 7-H), 6.71 / 6.94, 6.90 (7-H / 8-H, 6-H), 6.66 / 2.06,

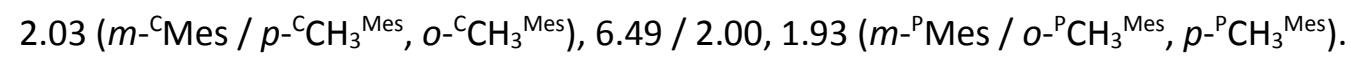

${ }^{1} \mathrm{H}^{13}{ }^{13} \mathrm{C} \mathrm{GHSQC}\left(600 \mathrm{MHz} / 151 \mathrm{MHz}, \mathrm{C}_{6} \mathrm{D}_{6}, 299 \mathrm{~K}\right): \delta^{1} \mathrm{H} / \delta^{13} \mathrm{C}=7.52$ / 129.8 (5-H / C5), 6.94 / 125.6 (8-H / C8), 6.90 / 128.5 (6-H / C6), 6.71 / 127.4 (7-H / C7), 6.66 / 129.3 ( - $^{\mathrm{C}}$ Mes / m- ${ }^{\mathrm{C}}$ Mes), 6.49 / 130.3 ( $m-{ }^{\mathrm{P}}$ Mes $/ m$ - ${ }^{\mathrm{P}}$ Mes) $, 2.06 / 21.0\left(p^{-}{ }^{\mathrm{C}} \mathrm{CH}_{3}{ }^{\text {Mes }} / p^{-}{ }^{\mathrm{C}} \mathrm{CH}_{3}{ }^{\text {Mes }}\right), 2.03 / 20.6\left(o^{-}{ }^{\mathrm{C}} \mathrm{CH}_{3}{ }^{\text {Mes }} / o_{-}{ }^{\mathrm{C}} \mathrm{CH}_{3}{ }^{\text {Mes }}\right), 2.00 / 22.5$ (o- $\left.{ }^{\mathrm{P}} \mathrm{CH}_{3}{ }^{\mathrm{Mes}} /{ }^{-}-{ }^{\mathrm{P}} \mathrm{CH}_{3}{ }^{\mathrm{Mes}}\right), 1.93 / 20.7\left(p-{ }^{\mathrm{P}} \mathrm{CH}_{3}{ }^{\mathrm{Mes}} / p-{ }^{\mathrm{P}} \mathrm{CH}_{3}{ }^{\mathrm{Mes}}\right),-0.02 / 1.9\left(\mathrm{SiCH}_{3} / \mathrm{SiCH}_{3}\right)$.

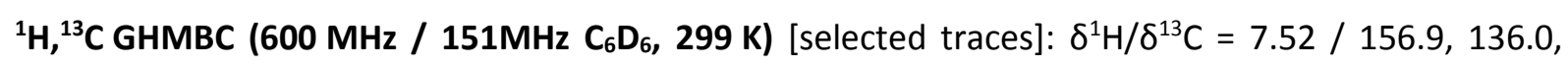
127.4 (5-H / C4, C8a, C7), 6.94 / 183.5, 138.4, 128.5 (8-H / C1, C4a, C6), 6.90 / 138.4, 125.6 (6-H / C4a, C8), 6.71 / 136.0, 129.8 (7-H / C8a, C5), 6.66 / 132.4, 129.3 ( $m$ - ${ }^{\mathrm{C}}$ Mes / $\left.{ }^{-}{ }^{\mathrm{C}} \mathrm{Mes},{ }^{\mathrm{C}}{ }^{\mathrm{C}} \mathrm{Mes}\right), 2.06$ / 138.1,

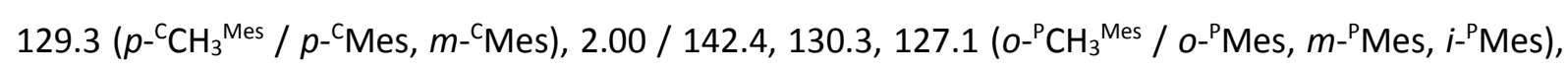
$1.93 / 142.2,130.3\left(p-{ }^{\mathrm{P}} \mathrm{CH}_{3}{ }^{\mathrm{Mes}} / p-{ }^{\mathrm{P}} \mathrm{Mes}, m-{ }^{\mathrm{P}} \mathrm{Mes}\right),-0.02 / 156.9,1.9\left(\mathrm{SiCH}_{3} / \mathrm{C} 4, \mathrm{SiCH}_{3}\right)$. 
${ }^{19} \mathrm{~F},{ }^{19} \mathrm{~F}$ GCOSY (564 MHz / $564 \mathrm{MHz}, \mathrm{C}_{6} \mathrm{D}_{6}, 299 \mathrm{~K}$ ) [selected traces]: $\delta^{19} \mathrm{~F} / \delta^{19} \mathrm{~F}=-125.8 /-160.0$ $\left(o-\mathrm{BC}_{6} \mathrm{~F}_{5} / m-\mathrm{BC}_{6} \mathrm{~F}_{5}\right),-126.4 /-163.0\left(o-\mathrm{PC}_{6} \mathrm{~F}_{5} / m-\mathrm{PC}_{6} \mathrm{~F}_{5}\right),-148.2 /-160.0\left(p-\mathrm{BC}_{6} \mathrm{~F}_{5} / m-\mathrm{BC}_{6} \mathrm{~F}_{5}\right),-154.9$ / $-163.0\left(p-\mathrm{PC}_{6} \mathrm{~F}_{5} / m-\mathrm{PC}_{6} \mathrm{~F}_{5}\right),-158.0 /-163.6\left(p-\mathrm{C}_{6} \mathrm{~F}_{5} / m-\mathrm{C}_{6} \mathrm{~F}_{5}\right)$.

${ }^{19} \mathrm{~F},{ }^{1} \mathrm{H}$ GHOESY (564 MHz / $600 \mathrm{MHz}, \mathrm{C}_{6} \mathrm{D}_{6}, 299 \mathrm{~K}$ ) [selected traces]: $\delta^{19} \mathrm{~F} / \delta^{1} \mathrm{H}=-125.8 / 2.00,-0.02$ $\left(0-\mathrm{BC}_{6} \mathrm{~F}_{5} / 0^{-}{ }^{\mathrm{P}} \mathrm{CH}_{3}{ }^{\mathrm{Mes}}, \mathrm{SiCH}_{3}\right),-126.4 / 6.94,2.00,-0.02\left(0-\mathrm{PC}_{6} \mathrm{~F}_{5} / 8-\mathrm{H}, 0^{-}{ }^{\mathrm{P}} \mathrm{CH}_{3}{ }^{\mathrm{Mes}}, \mathrm{SiCH}_{3}\right)$.

${ }^{1} \mathrm{H},{ }^{19} \mathrm{~F}$ GHOESY (600 MHz / $564 \mathrm{MHz}, \mathrm{C}_{6} \mathrm{D}_{6}, 299 \mathrm{~K}$ ) [selected traces]: $\delta^{19} \mathrm{~F} / \delta^{1} \mathrm{H}=6.94 /-126.4(8-\mathrm{H} /$ $\left.0-\mathrm{PC}_{6} \mathrm{~F}_{5}\right), 2.00 /-125.8,-126.4\left(0-{ }^{\mathrm{P}} \mathrm{CH}_{3}{ }^{\mathrm{Mes}} / 0-\mathrm{BC}_{6} \mathrm{~F}_{5}, 0-\mathrm{PC}_{6} \mathrm{~F}_{5}\right)$.

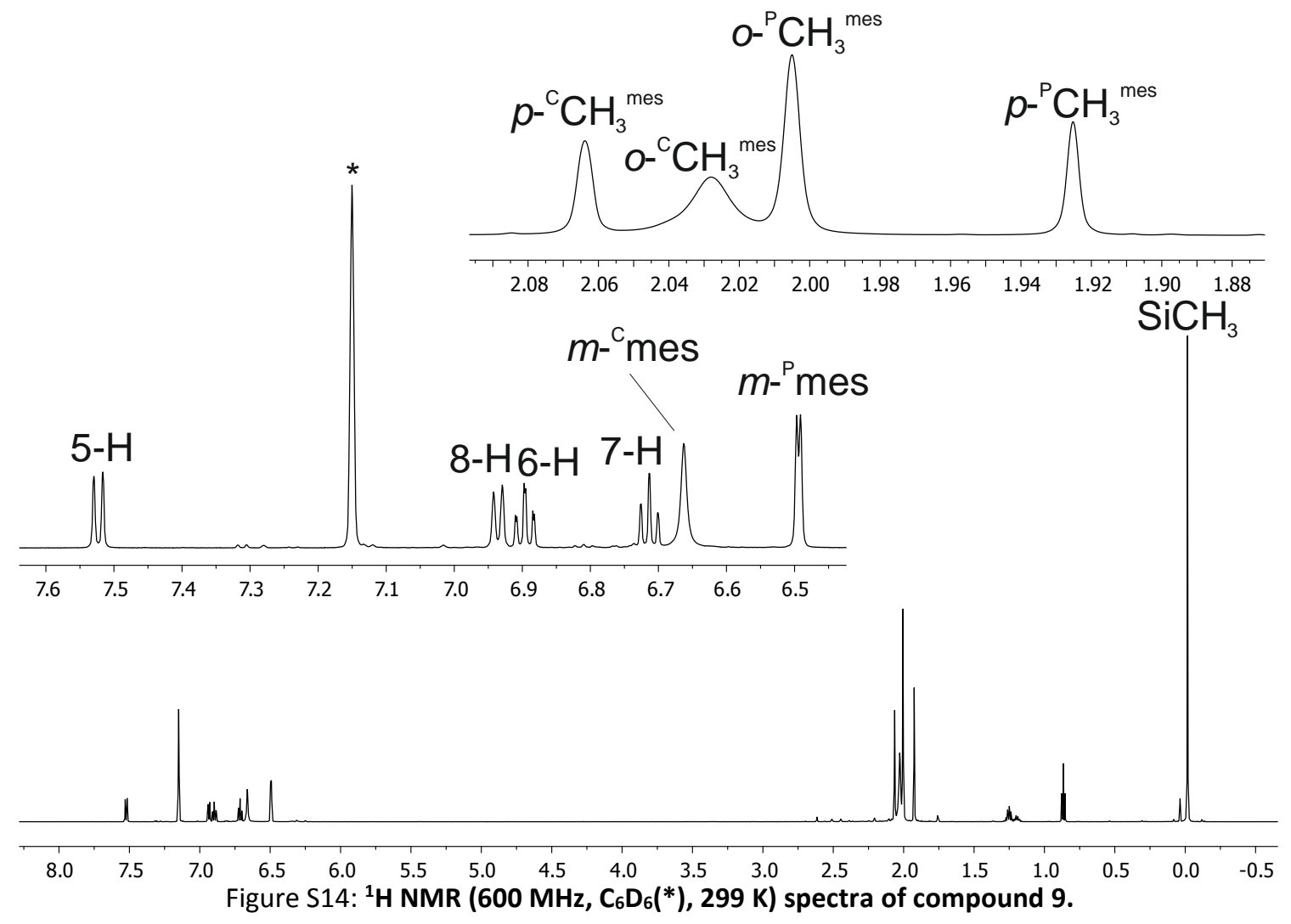




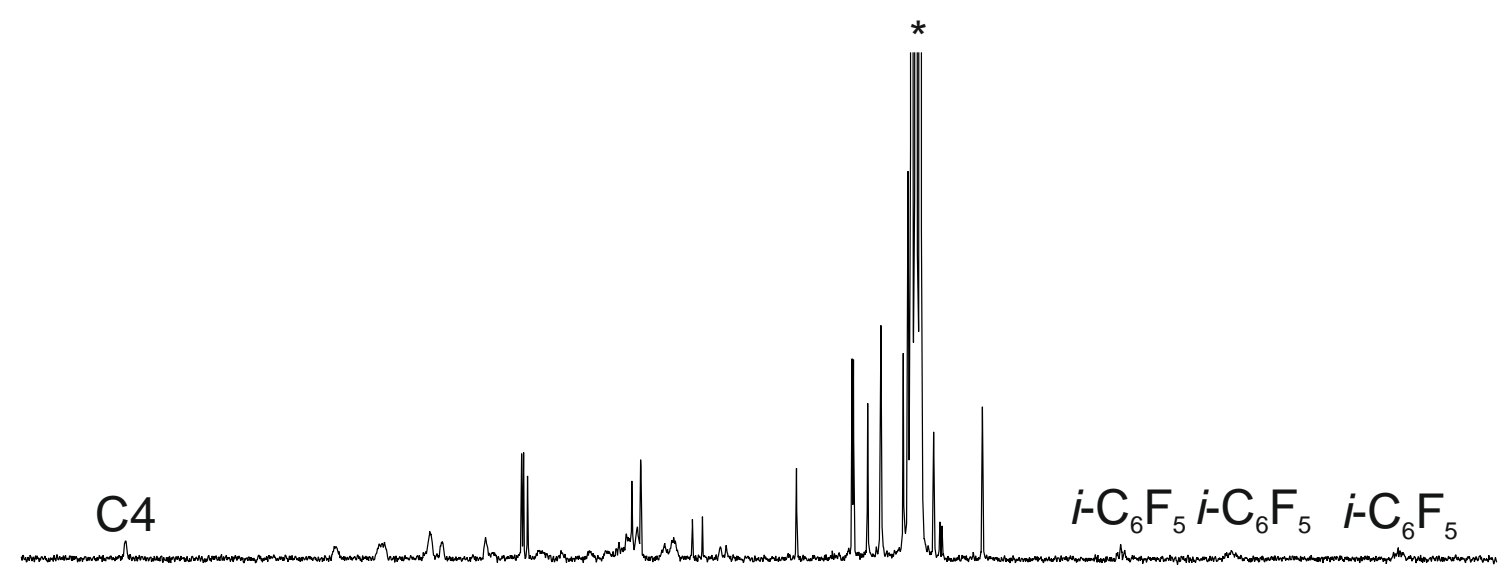

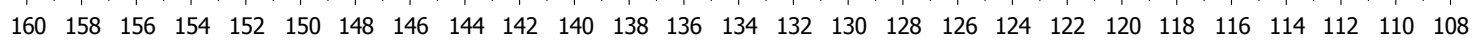
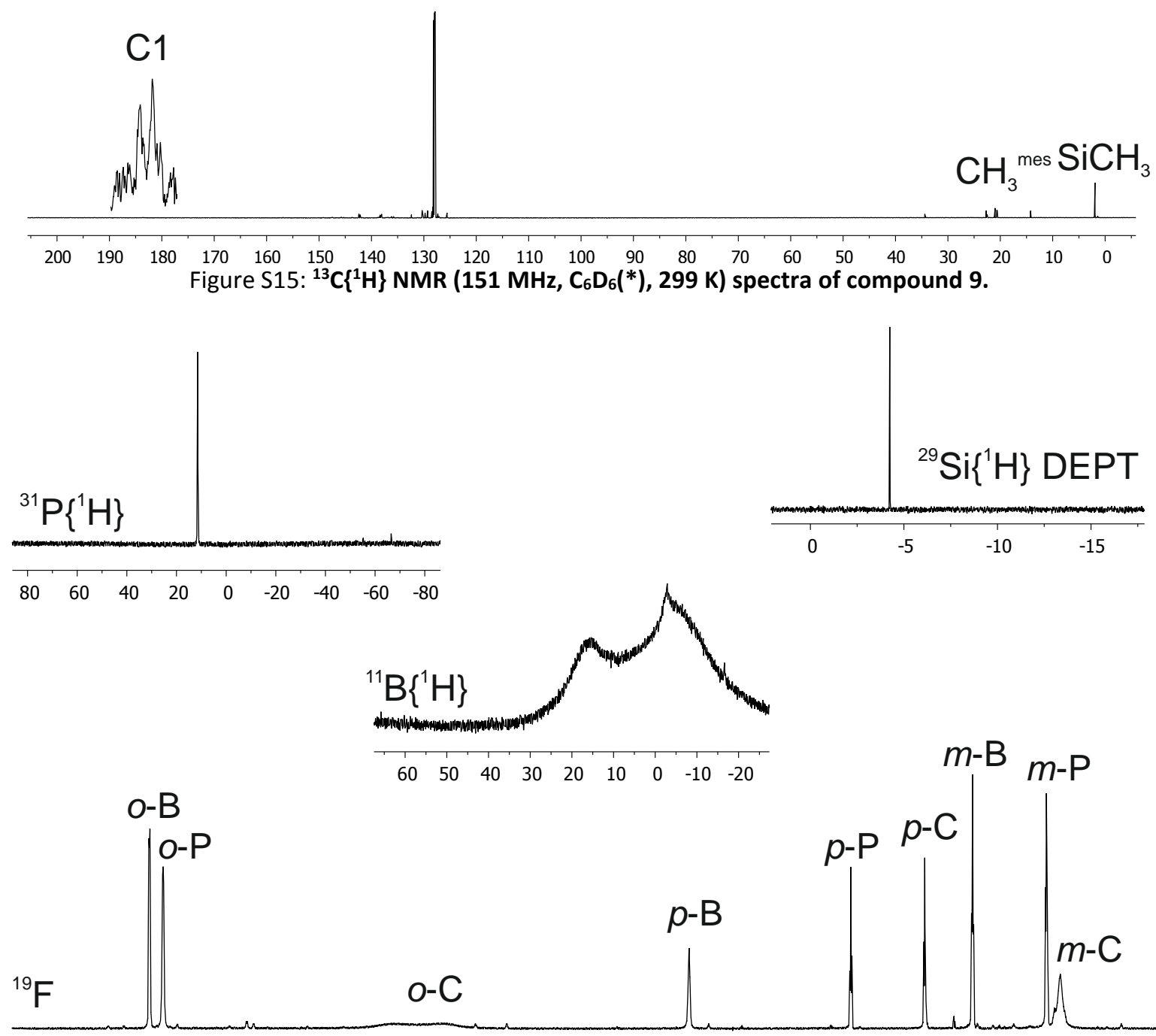

$\begin{array}{llllllllll}60 & 50 & 40 & 30 & 20 & 10 & 0 & -10 & -20\end{array}$

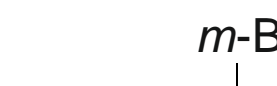

$m-\mathrm{B} m-\mathrm{P}$

$\begin{array}{lllllllllllllllllllllll}-122 & -124 & -126 & -128 & -130 & -132 & -134 & -136 & -138 & -140 & -142 & -144 & -146 & -148 & -150 & -152 & -154 & -156 & -158 & -160 & -162 & -164 & -166\end{array}$

Figure S16: ${ }^{19} \mathrm{~F}$ NMR (564 MHz, $\left.\mathrm{C}_{6} \mathrm{D}_{6}, 299 \mathrm{~K}\right),{ }^{11} \mathrm{~B}\left\{{ }^{1} \mathrm{H}\right\}$ NMR (192 MHz), ${ }^{31} \mathrm{P}\left\{{ }^{1} \mathrm{H}\right\}$ NMR (243 MHz) and ${ }^{29} \mathrm{Si}\left\{{ }^{1} \mathrm{H}\right\}$ DEPT (99 MHz) spectra of compound 9. 
Comment: please note, in the ${ }^{31} \mathrm{P},{ }^{1} \mathrm{H}$ ghmbc experiment the ${ }^{1} \mathrm{H}$ resonance of the para-methyl group of the carbon bonded mesityl group generates a cross peak to the ${ }^{31} \mathrm{P}$.

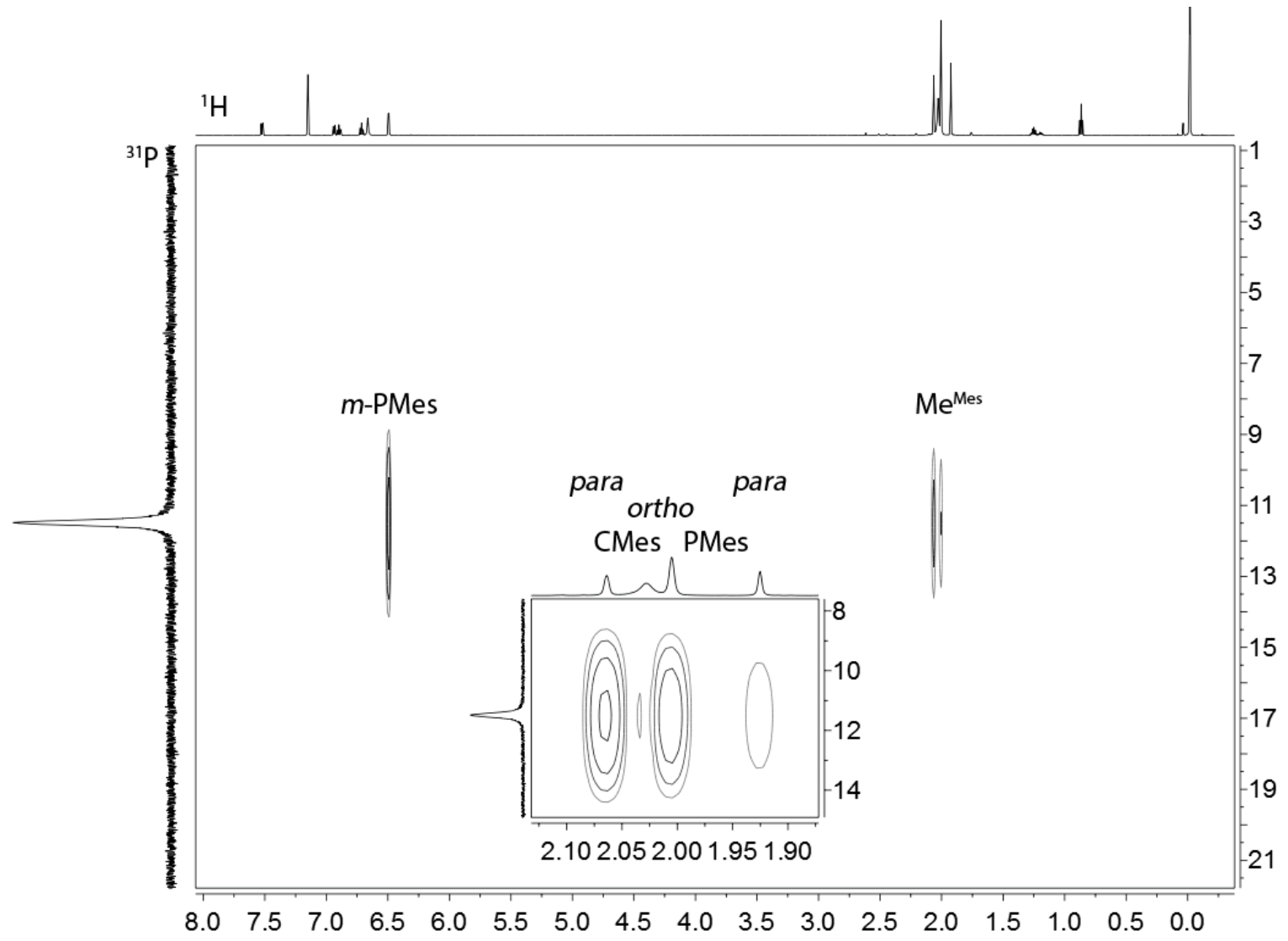

Figure S17: ${ }^{31} \mathrm{P},{ }^{1} \mathrm{H}$ ghmbc $\left(600,243 \mathrm{MHz}, \mathrm{C}_{6} \mathrm{D}_{6}, 299 \mathrm{~K}\right)$ spectra of compound 9

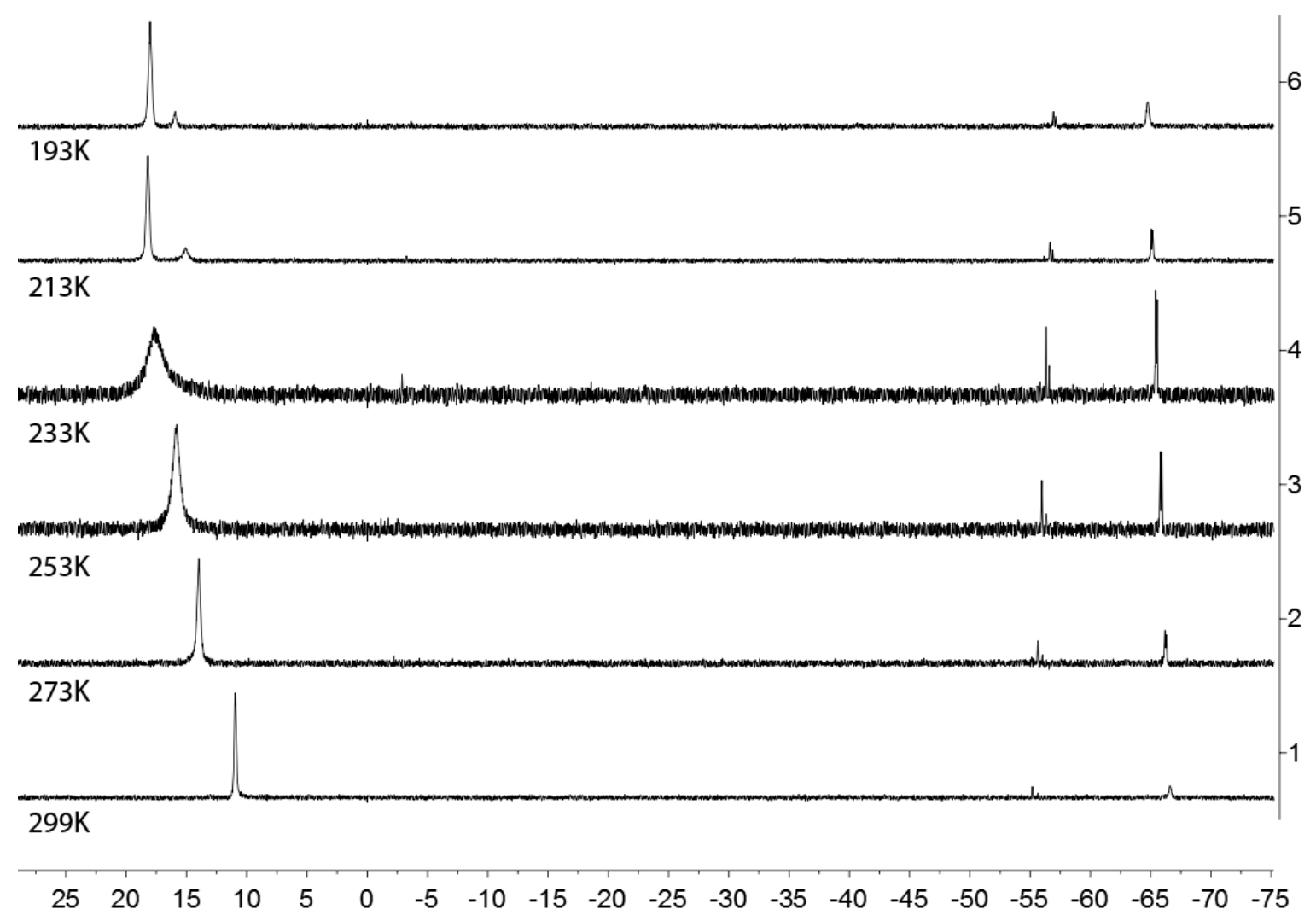

Figure S18: ${ }^{31} \mathrm{P}\left\{{ }^{1} \mathrm{H}\right\}$ NMR $\left(202 \mathrm{MHz}, \mathrm{C}_{7} \mathrm{D}_{8}\right)$ spectra of compound 9. 


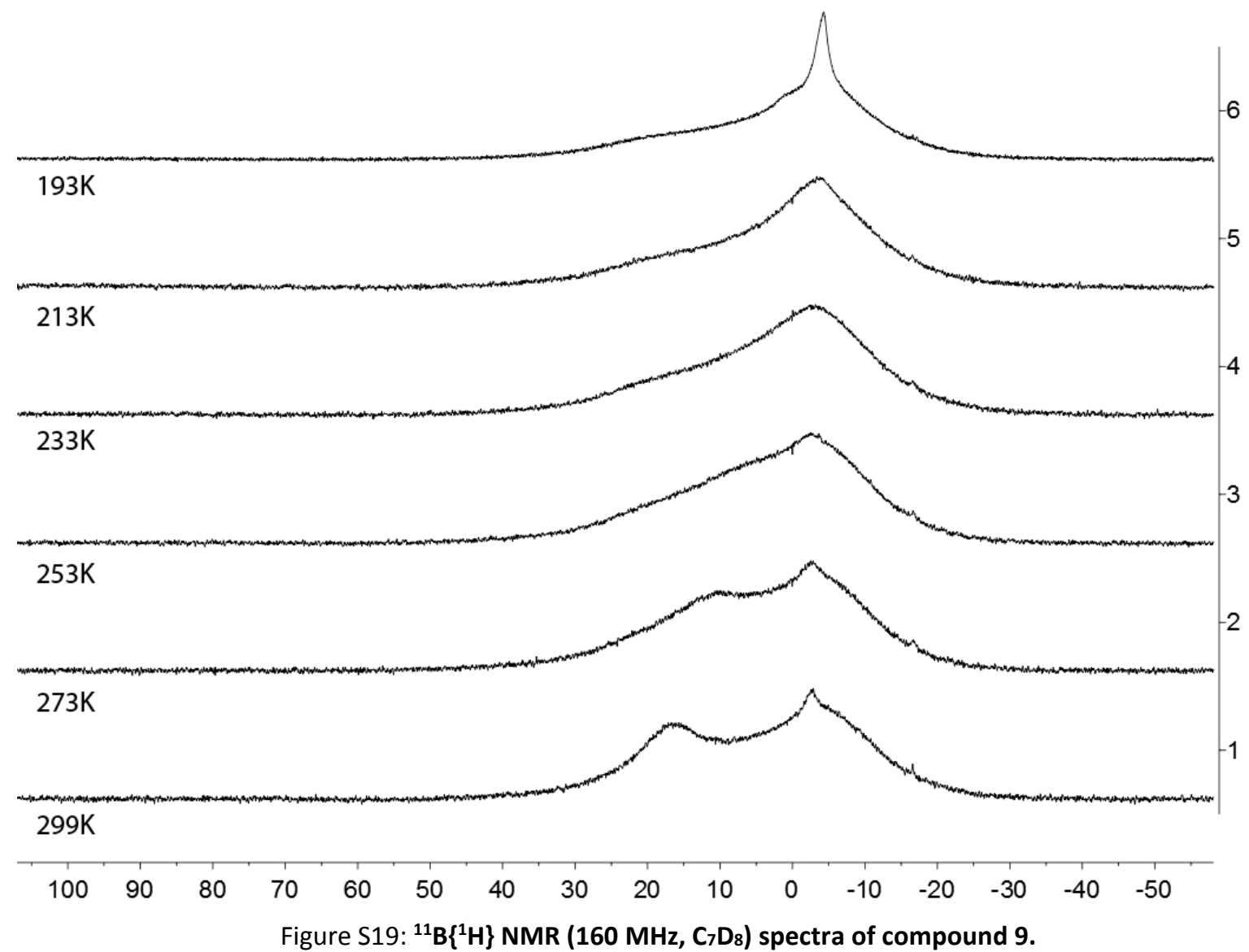

Figure S20: X-ray crystal structure analysis of compound 9: formula $\mathrm{C}_{49} \mathrm{H}_{35} \mathrm{BF}_{15} \mathrm{PSi}, M=978.64$, colourless crystal, $0.25 \times 0.10 \times 0.04 \mathrm{~mm}, a=11.2041(4), b=12.3549(3), c=18.8061(6) \AA, \alpha=$ 98.261(2), $\quad B=95.506(2), \gamma=103.061(1)^{\circ}, V=2487.2(1) \AA^{3}, \rho_{\text {calc }}=1.307 \mathrm{gcm}^{-3}, \mu=1.511 \mathrm{~mm}^{-1}$, empirical absorption correction (0.703 $\leq T \leq 0.942), Z=2$, triclinic, space group $P \overline{1}$ (No. 2$), \lambda=$ $1.54178 \AA, T=223(2) \mathrm{K}, \omega$ and $\phi$ scans, 33081 reflections collected $( \pm h, \pm k, \pm /),[(\sin \theta) / \lambda]=0.60 \AA^{-1}$, 8664 independent $\left(R_{\text {int }}=0.046\right)$ and 7307 observed reflections $[/>2 \sigma(/)], 613$ refined parameters, $R=$ $0.044, w R^{2}=0.124$, max. (min.) residual electron density $0.25(-0.30)$ e. $\AA^{-3}$, hydrogen atoms were calculated and refined as riding atoms. 


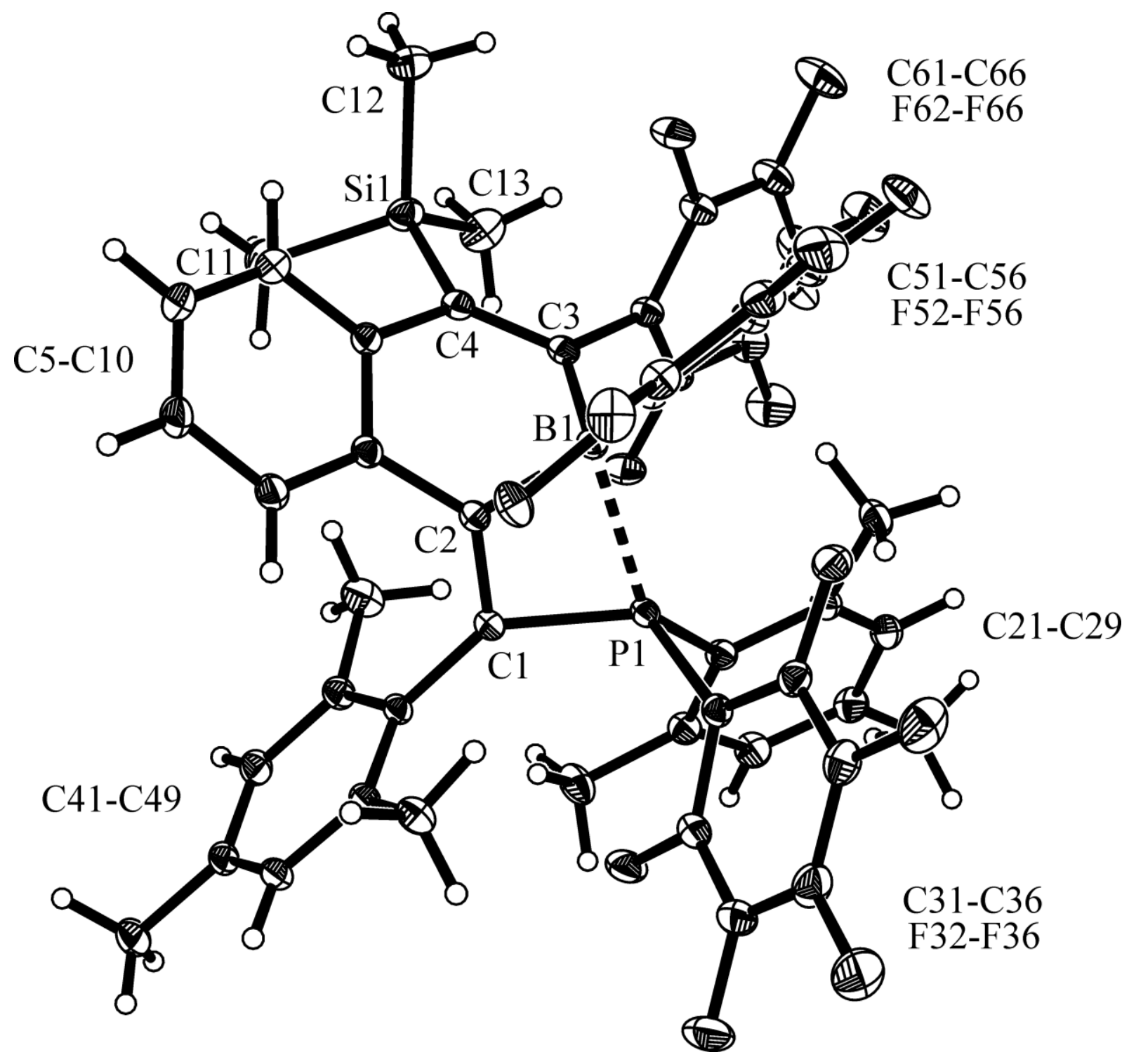




\section{Reaction with nitric oxide}

\section{Synthesis of compound 12}

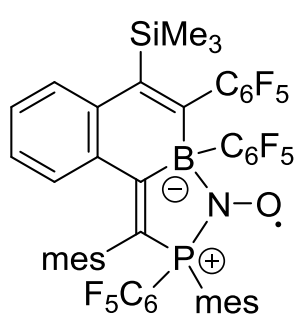

Scheme 57

Compound 9 ( $288 \mathrm{mg}, 0.29 \mathrm{mmol}, 1 \mathrm{eq}$ ) was dissolved in $\mathrm{C}_{6} \mathrm{H}_{6}(\sim 5 \mathrm{~mL})$ and the solution was exposed to 1.5 bar of NO gas. The color of the solution changed from red to yellow within a couple of minutes and the resulting solution was stirred for $2 \mathrm{~h}$ at room temperature. After that all volatiles were removed in vacuo and the resulting residue was redissolved in $n$-pentane $(\sim 5 \mathrm{~mL})$ and the time to remove remaining $\left.\mathrm{C}_{6} \mathrm{H}_{6}\right)$. Then the residue was redissolved in $n$-pentane $(\sim 5 \mathrm{~mL})$ and sonicated for $10 \mathrm{~min}$. The supernatant was removed and the residue washed with $n$-pentane $(2 \mathrm{x} \sim$ $3 \mathrm{~mL}$ ). Drying of the orange solid in vacuo gave compound 12 (190 mg, $0.19 \mathrm{mmol}$ ).

The washing solution was kept at $-40{ }^{\circ} \mathrm{C}$ for 3 days to give more orange material $(12$ (22.0 mg, $0.02 \mathrm{mmol})$ ), which was combined with the previous isolated material. In total compound 12 (212 $\mathrm{mg}, 0.21 \mathrm{mmol}, 72 \%$ ) was isolated as an orange solid. Single crystals suitable for the X-ray crystal structure analysis were obtained by slow diffusion of $n$-pentane into a solution of 12 in $\mathrm{CH}_{2} \mathrm{Cl}_{2}$ at $-40^{\circ} \mathrm{C}$.

IR (KBr): ṽ $\left[\mathrm{cm}^{-1}\right]=3442$ (br w), 2960 (br m), 2925 (br m), 1642 (m), 1605 (m), 1518 (s), 1486 (s), 1459 (s), $1397(w), 1383(w), 1303(m), 1253(m), 1152(w), 1101(s), 1029(w), 986(s), 909(m), 853(s)$, $771(w), 688(w), 658(m), 603(w), 580(w), 515(w)$.

Elemental analysis: Calcd for $\mathrm{C}_{49} \mathrm{H}_{35} \mathrm{BF}_{15} \mathrm{NOPSi}$ C, $58.35 \mathrm{H}, 3.50 \mathrm{~N}, 1.39$. Found: $\mathrm{C}, 58.57 \mathrm{H}, 3.62 \mathrm{~N}$, 1.41 .

Decomposition (DSC): $151^{\circ} \mathrm{C}$.

Figure S21: X-ray crystal structure analysis of compound 12: formula $\mathrm{C}_{49} \mathrm{H}_{35} \mathrm{BF}_{15} \mathrm{NOPSi}, M=1008.65$, yellow crystal, $0.18 \times 0.13 \times 0.05 \mathrm{~mm}, a=29.7467(7), b=16.9992(6), c=24.3490(7) \AA, b=$ $121.784(2)^{\circ}, V=10466.2(1) \AA^{3}, \rho_{\text {calc }}=1.280 \mathrm{gcm}^{-3}, \mu=1.473 \mathrm{~mm}^{-1}$, empirical absorption correction (0.777 $\leq T \leq 0.930, Z=8$, monoclinic, space group $C 2 / c$ (No. 15), $\lambda=1.54178 \AA, T=223(2) \mathrm{K}, \omega$ and $\phi$ scans, 41330 reflections collected $( \pm h, \pm k, \pm l),[(\sin \theta) / \lambda]=0.60 \AA^{-1}, 8712$ independent $\left(R_{\text {int }}=0.065\right)$ and 5713 observed reflections $[/>2 \sigma(I)], 631$ refined parameters, $R=0.059, w R^{2}=0.175$, max. (min.) residual electron density $0.30(-0.27)$ e. $\AA^{-3}$, hydrogen atoms were calculated and refined as riding atoms. 


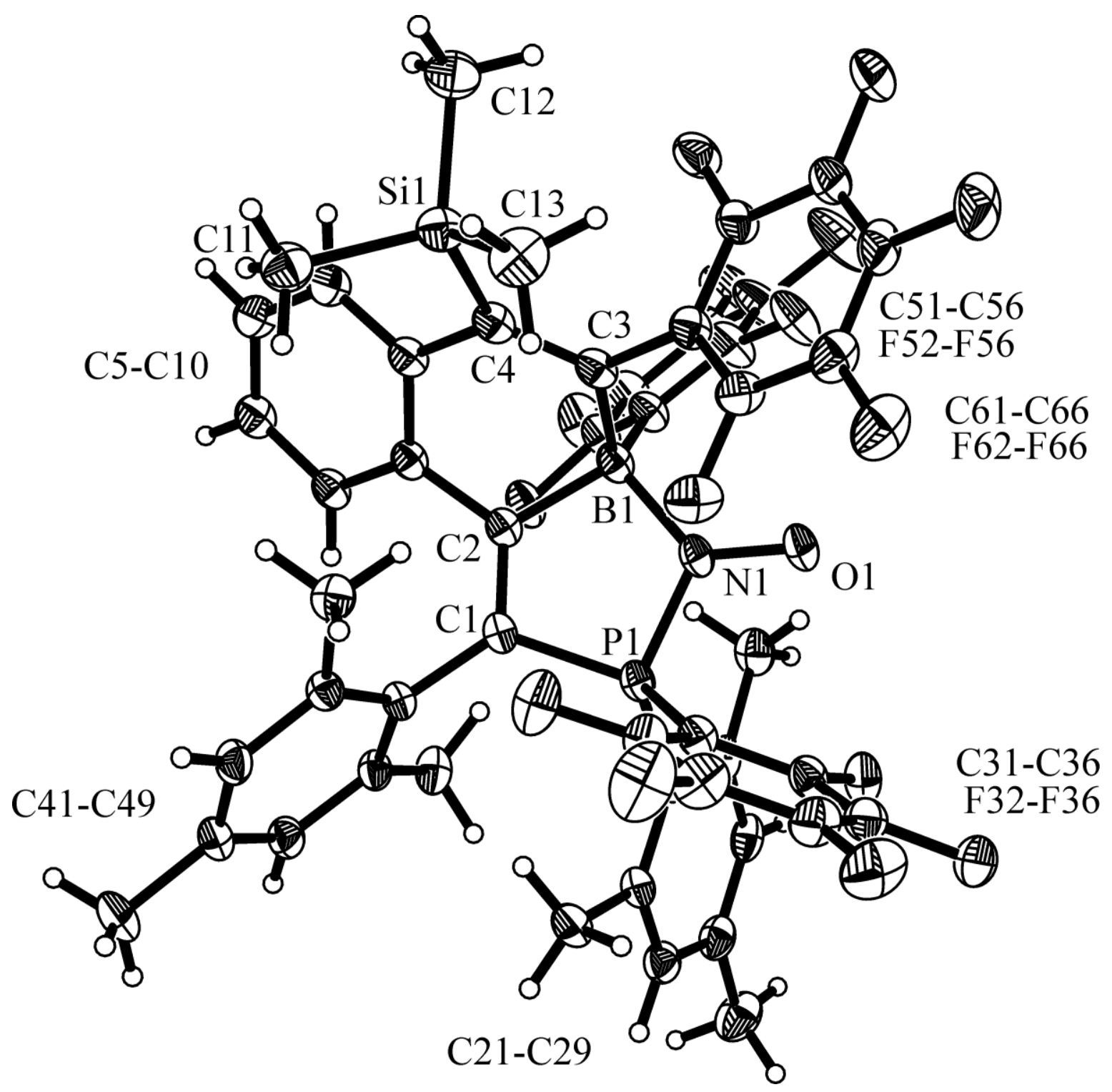

EPR Spectroscopy

The EPR measurement was performed in quartz tubes with J. Young valves. Solution EPR spectra were recorded on a JEOL continuous wave spectrometer JES-FA200 equipped with an X-band Gunn oscillator bridge and a cylindrical mode cavity. For all samples, a modulation frequency of $100 \mathrm{kHz}$ was employed. Spectrum was obtained from freshly prepared benzene solution of compound 12 at $298 \mathrm{~K}$.

Spectral simulation was performed using the program QCMP 136 by Prof. Dr. Frank Neese from the Quantum Chemistry Program Exchange as used by Neese et al. in J. Am. Chem. Soc. 1996, 118, 8692-8699. The fittings were performed by the "chi by eye" approach. Collinear $g$ and $A$ tensors were used. 
Figure S22: X-band EPR spectrum and simulation for compound 12 (benzene, RT): $8.89010 \mathrm{GHz}$, ModWidth $=0.01 \mathrm{mT}$, Power $=1.0 \mathrm{~mW}$, time constant $=1.0 \mathrm{~s}$, Simulation (Gaussian lineshape with $1.4 \mathrm{G}$ linebroadening) gives $g_{\text {iso }}=2.0081, A\left({ }^{14} \mathrm{~N}\right)=18.5 \mathrm{MHz}, A\left({ }^{31} \mathrm{P}\right)=48.9 \mathrm{MHz}, A\left({ }^{11} \mathrm{~B}\right)=9.7 \mathrm{MHz}$. Coupling to ${ }^{10} \mathrm{~B}$ was not simulated.

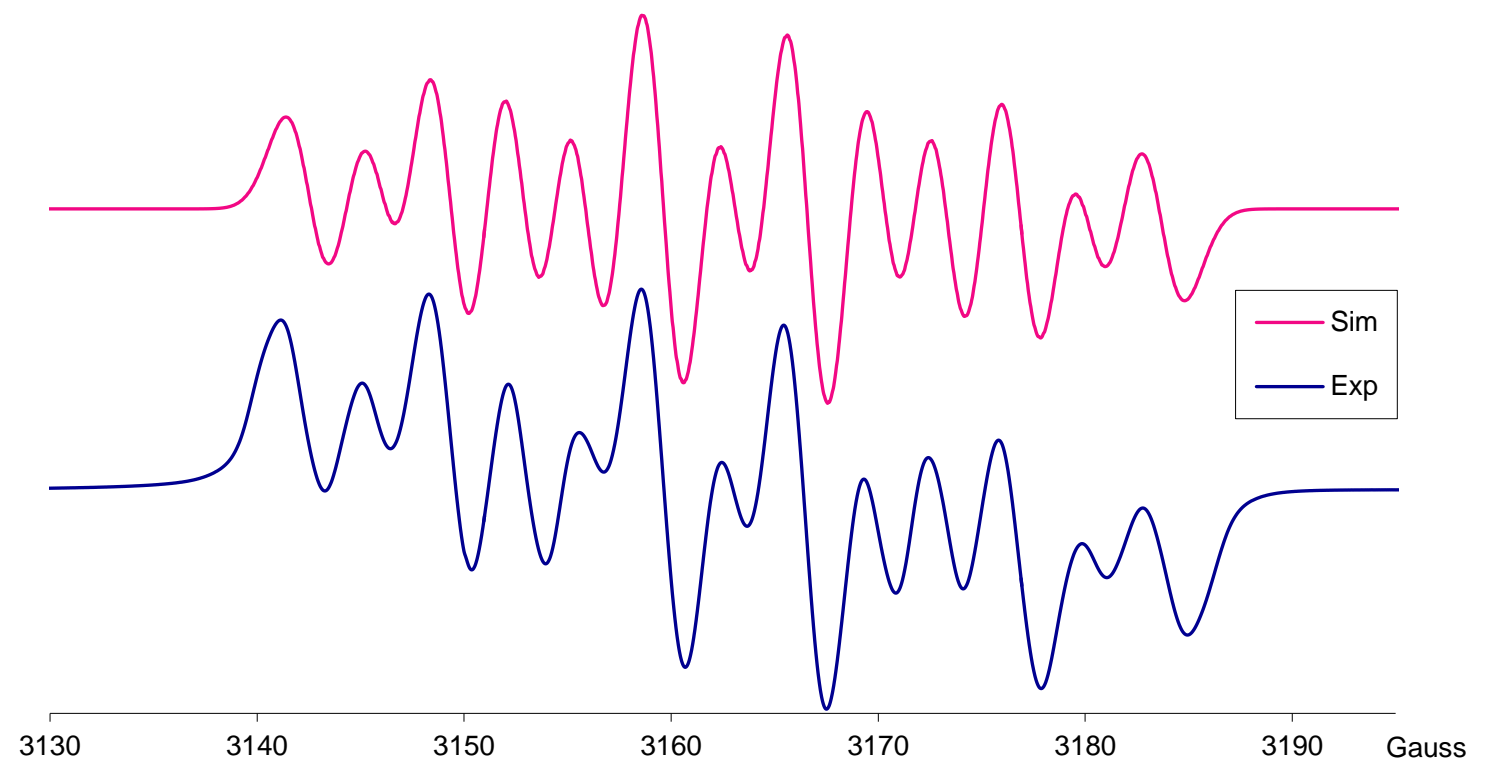




\section{Synthesis of compound 13}

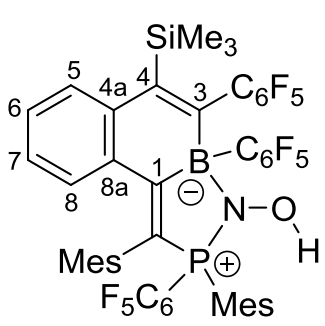

Scheme S8

Compound 12 (101 mg, $0.1 \mathrm{mmol}, 1 \mathrm{eq})$ was dissolved in $\mathrm{C}_{6} \mathrm{H}_{6}(\sim 3 \mathrm{~mL})$ and 1,4-cyclohexadiene ( $20 \mu \mathrm{L}, 0.2 \mathrm{mmol}, 2 \mathrm{eq})$ was added. The resulting solution was stirred at room temperature overnight, then all volatiles were removed in vacuo and $n$-pentane $(\sim 5 \mathrm{~mL}$ ) was added and directly removed in vacuo (to remove remaining $\left.\mathrm{C}_{6} \mathrm{H}_{6}\right)$. The residue was purified by flash-chromatography $\left(\mathrm{SiO}_{2}, \mathrm{CH}_{2} \mathrm{Cl}_{2}: n\right.$-pentane $\left.=1: 4\right)$. After drying in vacuo compound $13(94.0 \mathrm{mg}$,

$0.09 \mathrm{mmol}, 94 \%)$ was isolated as a colorless solid. Single crystals suitable for the X-ray crystal structure analysis were obtained from a saturated solution of compound $\mathbf{1 3}$ in $\mathrm{C}_{6} \mathrm{D}_{6}$ at room temperature.

IR (ATR): $\tilde{v}\left[\mathrm{~cm}^{-1}\right]=3543(\mathrm{w}), 2956(\mathrm{br} \mathrm{m}), 2926(\mathrm{br} \mathrm{m}), 1643(\mathrm{~m}), 1606(\mathrm{~m}), 1516(\mathrm{~s}), 1485(\mathrm{~s}), 1455$ (s), $1383(w), 1300(w), 1253(m), 1150(w), 1100(s), 1016(m), 984(s), 917(m), 845(s), 768(m), 685$ $(w), 656(m), 604(w), 514(w)$.

Elemental analysis: Calcd for $\mathrm{C}_{49} \mathrm{H}_{36} \mathrm{BF}_{15} \mathrm{NOPSi}$ : $\mathrm{C}, 58.29 \mathrm{H}, 3.59 \mathrm{~N}, 1.29$. Found: $\mathrm{C}, 58.16 \mathrm{H}, 4.08 \mathrm{~N}$, 1.24 .

Decomposition (DSC): $227^{\circ} \mathrm{C}$.

${ }^{1}{ }_{H}$ NMR $\left(600 \mathrm{MHz}, \mathrm{C}_{6} \mathrm{D}_{6}, 299 \mathrm{~K}\right): \delta=7.35(\mathrm{~m}, 1 \mathrm{H}, 5-\mathrm{H}), 6.91(\mathrm{~m}, 1 \mathrm{H}, 8-\mathrm{H}), 6.90(\mathrm{~m}, 1 \mathrm{H}, 6-\mathrm{H}), 6.73(\mathrm{~m}$, $1 \mathrm{H}, 7-\mathrm{H}), 6.64\left(\mathrm{~s}, 1 \mathrm{H}, m^{\prime}-{ }^{\mathrm{C}} \mathrm{Mes}\right), 6.63\left(\mathrm{~s}, 1 \mathrm{H}, m^{-}{ }^{\mathrm{C}} \mathrm{Mes}\right), 6.42\left(\mathrm{~d},{ }^{4} \mathrm{~J}_{\mathrm{PH}}=4.5 \mathrm{~Hz}, 1 \mathrm{H}, m^{\prime}-{ }^{\mathrm{P}} \mathrm{Mes}\right), 6.34\left(\mathrm{~d},{ }^{4} J_{\mathrm{PH}}\right.$ $\left.=4.5 \mathrm{~Hz}, 1 \mathrm{H}, m^{\mathrm{P}} \mathrm{Mes}\right), 3.54\left(\mathrm{dm},{ }^{3} J_{\mathrm{PH}}=5.9 \mathrm{~Hz}, 1 \mathrm{H}, \mathrm{NOH}\right), 2.06\left(\mathrm{~d}, J=8.1 \mathrm{~Hz}, 3 \mathrm{H}, o^{\prime}-{ }^{\mathrm{C}} \mathrm{CH}_{3}{ }^{\mathrm{Mes}}\right), 2.05(\mathrm{~m}$, $\left.3 \mathrm{H}, p-{ }^{\mathrm{C}} \mathrm{CH}_{3}{ }^{\mathrm{Mes}}\right), 1.94\left(\mathrm{~s}, 3 \mathrm{H}, o^{\prime}-{ }^{\mathrm{P}} \mathrm{CH}_{3}{ }^{\text {Mes }}\right), 1.88\left(\mathrm{~s}, 3 \mathrm{H}, p-{ }^{\mathrm{P}} \mathrm{CH}_{3}{ }^{\mathrm{Mes}}\right), 1.74\left(\mathrm{~s}, 3 \mathrm{H}, o^{-}{ }^{\mathrm{C}} \mathrm{CH}_{3}{ }^{\mathrm{Mes}}\right), 1.36(\mathrm{~s}, 3 \mathrm{H}$, $\left.O-{ }^{\mathrm{P}} \mathrm{CH}_{3}{ }^{\mathrm{Mes}}\right),-0.01\left(\mathrm{~s}^{2} \mathrm{~J}_{\mathrm{SiH}}=6.0 \mathrm{~Hz}, \mathrm{SiCH}_{3}\right)$.

${ }^{1} \mathrm{H}\left\{{ }^{31} \mathrm{P}\right\}$ NMR (600 MHz, $\mathrm{C}_{6} \mathrm{D}_{6}, 299 \mathrm{~K}$ ) [selected resonances]: $\delta=6.42\left(\mathrm{~s}, 1 \mathrm{H}, \mathrm{m}^{\prime}{ }^{-}{ }^{\mathrm{P}} \mathrm{Mes}\right), 6.34(\mathrm{~s}, 1 \mathrm{H}$, $\left.m-{ }^{\mathrm{P}} \mathrm{Mes}\right), 3.54(\mathrm{br}, 1 \mathrm{H}, \mathrm{NOH}), 2.06\left(\mathrm{~d}, J=8.1 \mathrm{~Hz}, 3 \mathrm{H}, o^{\prime}-{ }^{\mathrm{C}} \mathrm{CH}_{3}{ }^{\mathrm{Mes}}\right), 2.06$ (s, 3H, $p-{ }^{\mathrm{C}} \mathrm{CH}_{3}{ }^{\mathrm{Mes}}$ ).

${ }^{13} \mathrm{C}\left\{{ }^{1} \mathrm{H}\right\}$ NMR (151 MHz, $\left.\mathrm{C}_{6} \mathrm{D}_{6}, 299 \mathrm{~K}\right): \delta=189.0(\mathrm{br}, \mathrm{C} 1), 152.1(\mathrm{br}, \mathrm{C} 3)^{\mathrm{t}}, 149.1(\mathrm{C} 4), 143.8\left(\mathrm{~d},{ }^{4} J_{\mathrm{PC}}=\right.$ $3.1 \mathrm{~Hz}, p-{ }^{\mathrm{P}} \mathrm{Mes}$ ), 142.2 (d, $\left.{ }^{2} J_{\mathrm{PC}}=9.4 \mathrm{~Hz}, o^{\prime}-{ }^{\mathrm{P}} \mathrm{Mes}\right), 141.0$ (d, ${ }^{2} J_{\mathrm{PC}}=13.9 \mathrm{~Hz}, o^{\mathrm{P}}{ }^{\mathrm{Mes}}$ ), 140.5 (C4a), 140.0 $\left(\mathrm{d},{ }^{3} \mathrm{JPC}_{\mathrm{PC}}=5.6 \mathrm{~Hz}, o^{\prime}-{ }^{\mathrm{C}} \mathrm{Mes}\right), 139.8$ (d, $\left.{ }^{3} \mathrm{JPC}_{\mathrm{PC}}=2.8 \mathrm{~Hz}, \mathrm{o}^{\mathrm{C}}{ }^{\mathrm{C}} \mathrm{Mes}\right), 138.0$ (d, $\left.{ }^{5} \mathrm{PC}_{\mathrm{PC}}=2.4 \mathrm{~Hz}, p^{-}{ }^{\mathrm{C}} \mathrm{Mes}\right), 137.1\left(\mathrm{~d},{ }^{3} \mathrm{JPC}_{\mathrm{PC}}\right.$ $=22.0 \mathrm{~Hz}, \mathrm{C} 8 \mathrm{a}$ ), 131.9 (d, $\left.{ }^{3} \mathrm{~J}_{\mathrm{PC}}=10.6 \mathrm{~Hz}, m^{\prime}-^{\mathrm{P} M e s}\right), 131.5$ (d, $\left.{ }^{3} J_{\mathrm{PC}}=10.6 \mathrm{~Hz}, m^{\mathrm{P}}{ }^{\mathrm{P}} \mathrm{Mes}\right), 129.9$ ( $m^{\prime}-{ }^{\mathrm{C}}$ Mes),

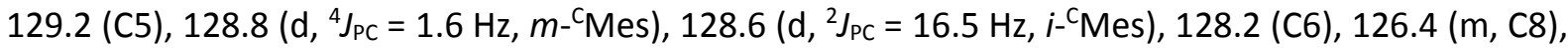
$126.2(\mathrm{C} 7), 124.1$ (d, ${ }^{1} J_{\mathrm{PC}}=90.1 \mathrm{~Hz}, i-{ }^{\mathrm{P}}$ Mes), $117.3\left(\mathrm{~d},{ }^{1} J_{\mathrm{PC}}=100.5 \mathrm{~Hz},=\mathrm{CP}\right.$ ) ${ }^{\mathrm{t}}, 23.2\left(\mathrm{~d},{ }^{3} J_{\mathrm{PC}}=5.4 \mathrm{~Hz}\right.$, $\left.0-{ }^{\mathrm{P}} \mathrm{CH}_{3}{ }^{\text {Mes }}\right), 20.9\left(\mathrm{~m}, p^{-}{ }^{\mathrm{C}} \mathrm{CH}_{3}{ }^{\text {Mes }}\right), 20.8\left(\mathrm{~d},{ }^{5} \mathrm{JPC}_{\mathrm{PC}}=1.4 \mathrm{~Hz}, p-{ }^{\mathrm{P}} \mathrm{CH}_{3}{ }^{\text {Mes }}\right), 20.6\left({ }^{-}{ }^{\mathrm{C}} \mathrm{CH}_{3}{ }^{\text {Mes }}\right), 20.1(\mathrm{~d}, J=19.7 \mathrm{~Hz}$, $\left.o^{\prime}-{ }^{\mathrm{C}} \mathrm{CH}_{3}{ }^{\text {Mes }}\right), 19.4\left(\mathrm{~d},{ }^{3} \mathrm{~J}_{\mathrm{PC}}=5.7 \mathrm{~Hz}, \mathrm{o}^{\prime}-{ }^{\mathrm{P}} \mathrm{CH}_{3}{ }^{\mathrm{Mes}}\right), 1.7\left({ }^{1} \mathrm{~J}_{\mathrm{SiC}}=52.4 \mathrm{~Hz}, \mathrm{SiCH}_{3}\right),\left[\mathrm{C}_{6} \mathrm{~F}_{5}\right.$ not listed, ${ }^{\mathrm{t}}$ tentative assignment].

${ }^{31} \mathrm{P}\left\{{ }^{1} \mathrm{H}\right\}$ NMR $\left(243 \mathrm{MHz}, \mathrm{C}_{6} \mathrm{D}_{6}, 299 \mathrm{~K}\right): \delta=38.1\left(\mathrm{v}_{1 / 2}=30.0 \mathrm{~Hz}\right)$.

${ }^{10} \mathrm{~B}\left\{{ }^{1} \mathrm{H}\right\}$ NMR $\left(64 \mathrm{MHz}, \mathrm{C}_{6} \mathrm{D}_{6}, 299 \mathrm{~K}\right): \delta=-4.4\left(\mathrm{~V}_{1 / 2} \sim 200 \mathrm{~Hz}\right)$. 
${ }^{29} \mathrm{Si}\left\{{ }^{1} \mathrm{H}\right\}$ DEPT (119 MHz, $\left.\mathrm{C}_{6} \mathrm{D}_{6}, 299 \mathrm{~K}\right): \delta=-5.7\left(\mathrm{v}_{1 / 2} \sim 2 \mathrm{~Hz}\right)$.

${ }^{19} \mathrm{~F}$ NMR $\left(564 \mathrm{MHz}, \mathrm{C}_{6} \mathrm{D}_{6}, 299 \mathrm{~K}\right): \delta=-123.2(\mathrm{~m}, o),-126.2\left(\mathrm{~m}, o^{\prime}\right),-146.0\left(\mathrm{t},{ }^{3} \mathrm{JFF}_{\mathrm{FF}}=20.9 \mathrm{~Hz}, p\right),-159.7$ $\left(\mathrm{m}, m^{\prime}\right),-160.9(\mathrm{~m}, m)\left(1 \mathrm{~F}, \mathrm{PC}_{6} \mathrm{~F}_{5}\right),\left[\Delta \delta^{19} \mathrm{~F}_{m, p}=14.9,13.7\right],-138.3(\mathrm{~m}, o),-139.9\left(\mathrm{~m}, o^{\prime}\right),-159.2\left(\mathrm{t},{ }^{3} J_{\mathrm{FF}}=\right.$ $21.4 \mathrm{~Hz}, p),-164.1(\mathrm{~m}, m),-168.0\left(\mathrm{~m}, \mathrm{~m}^{\prime}\right)\left(\right.$ each $\left.1 \mathrm{~F}, \mathrm{C}_{6} \mathrm{~F}_{5}\right),\left[\Delta \delta^{19} \mathrm{~F}_{m, p}=8.8,4.9\right],-125.3(\mathrm{br}, 2 \mathrm{~F}, o)$, $-158.1\left(\mathrm{t},{ }^{3} J_{\mathrm{FF}}=21.2 \mathrm{~Hz}, 1 \mathrm{~F}, p\right),-163.6(\mathrm{~m}, 2 \mathrm{~F}, m)\left(\mathrm{BC}_{6} \mathrm{~F}_{5}\right),\left[\Delta \delta^{19} \mathrm{~F}_{m, p}=5.5\right]$.

${ }^{1} H\left\{{ }^{1} H\right\}$ TOCSY (600 MHz, $C_{6} D_{6}, 299 K$ ) [selected experiments]: $\delta^{1} H_{\text {irr }} / \delta^{1} H_{\text {res }}=7.35 / 6.91,6.90,6.73$ (5-H / 8-H, 6-H, 7-H), 1.94 / 6.42, 6.34, 1.88, 1.36 (o'- ${ }^{\mathrm{P}} \mathrm{CH}_{3}{ }^{\text {Mes }} / m^{\prime}-{ }^{\mathrm{P}}$ Mes, $\left.m-{ }^{\mathrm{P}} \mathrm{Mes}, p-{ }^{\mathrm{P}} \mathrm{CH}_{3}{ }^{\mathrm{Mes}}, o^{-}{ }^{\mathrm{P}} \mathrm{CH}_{3}{ }^{\mathrm{Mes}}\right)$, 1.74 / 6.64, 6.63, 2.06, $2.06\left(o_{-}{ }^{C} \mathrm{CH}_{3}{ }^{\text {Mes }} / m^{\prime}-{ }^{C}\right.$ Mes, $m-{ }^{C}$ Mes, $\left.o^{\prime}-{ }^{C} \mathrm{CH}_{3}{ }^{\text {Mes }}, p^{-}{ }^{C} \mathrm{CH}_{3}{ }^{\text {Mes }}\right)$.

${ }^{1} \mathrm{H}\left\{{ }^{1} \mathrm{H}\right\}$ NOE (600 MHz, $\mathrm{C}_{6} \mathrm{D}_{6}, 299 \mathrm{~K}$ ) [selected experiments]: $\delta^{1} \mathrm{H}_{\text {irr }} / \delta^{1} \mathrm{H}_{\text {res }}=7.35 / 6.90,-0.01(5-\mathrm{H} /$ 6-H, $\left.\mathrm{SiCH}_{3}\right), 6.42$ / 1.94, 1.88 ( $m^{\prime}--^{\mathrm{P}}$ Mes / $\left.o^{\prime}-{ }^{\mathrm{P}} \mathrm{CH}_{3}{ }^{\mathrm{Mes}}, p^{\mathrm{P}} \mathrm{CH}_{3}{ }^{\mathrm{Mes}}\right), 6.34 / 1.88,1.36\left(m^{-}{ }^{\mathrm{P}} \mathrm{Mes} / p-{ }^{\mathrm{P}} \mathrm{CH}_{3}{ }^{\mathrm{Mes}}\right.$,

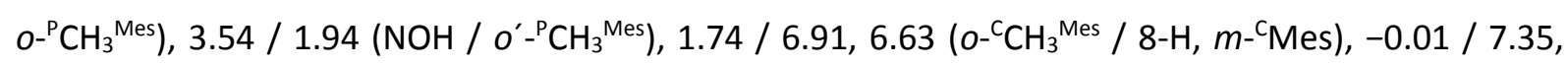
$2.06\left(\mathrm{SiCH}_{3} / 5-\mathrm{H}, \mathrm{O}^{\prime}-{ }^{C} \mathrm{CH}_{3}{ }^{\text {Mes }}\right)$.

${ }^{1} \mathrm{H}^{1}{ }^{1} \mathrm{H}$ GCOSY (600 MHz / $600 \mathrm{MHz}, \mathrm{C}_{6} \mathrm{D}_{6}, 299 \mathrm{~K}$ ) [selected traces]: $\delta^{1} \mathrm{H} / \delta^{1} \mathrm{H}=6.73 / 6.91,6.90(7-\mathrm{H} /$ 8-H, 6-H), 6.42 / 6.34, 1.94, 1.88, 1.36 ( $\left.m^{\prime}-{ }^{\mathrm{P}} \mathrm{Mes} / m^{\mathrm{P}} \mathrm{Mes}, o^{\prime}-{ }^{\mathrm{P}} \mathrm{CH}_{3}{ }^{\mathrm{Mes}}, p^{\mathrm{P}} \mathrm{CH}_{3}{ }^{\mathrm{Mes}}, o^{-}{ }^{\mathrm{P}} \mathrm{CH}_{3}{ }^{\mathrm{Mes}}\right)$.

${ }^{1} \mathrm{H}^{13}{ }^{13} \mathrm{C} \mathrm{GHSQC}\left(600 \mathrm{MHz} / 151 \mathrm{MHz}, \mathrm{C}_{6} \mathrm{D}_{6}, 299 \mathrm{~K}\right): \delta^{1} \mathrm{H} / \delta^{13} \mathrm{C}=7.35 / 129.2$ (5-H / C5), $6.91 / 126.5$ (8-H / C8), 6.90 / 128.3 (6-H / C6), 6.73 / 126.2 (7-H / C7), 6.64 / 129.9 ( $\mathrm{m}^{\prime}{ }^{-}{ }^{\mathrm{C}}$ Mes / $\left.\mathrm{m}^{\prime}{ }^{\mathrm{C}} \mathrm{Mes}\right), 6.63$ / 128.8 ( $m$ - ${ }^{\mathrm{C}}$ Mes / $m$ - ${ }^{\mathrm{C}}$ Mes), 6.42 / 131.9 ( $m^{\prime}{ }^{-}{ }^{\mathrm{P}}$ Mes / $m^{\prime}{ }^{\mathrm{P}}$ Mes), $6.34 / 131.5$ ( $m$ - $^{\mathrm{P}}$ Mes / m- ${ }^{\mathrm{P}}$ Mes), $2.06 / 20.1$ (o'- $\left.{ }^{\mathrm{C}} \mathrm{CH}_{3}{ }^{\text {Mes }} / o^{\prime}-{ }^{-} \mathrm{CH}_{3}{ }^{\text {Mes }}\right), 2.06 / 20.9\left(p^{-}{ }^{C} \mathrm{CH}_{3}{ }^{\text {Mes }} / p^{-}{ }^{C} \mathrm{CH}_{3}{ }^{\text {Mes }}\right), 1.94 / 19.4\left(o^{\prime}-{ }^{\mathrm{P}} \mathrm{CH}_{3}{ }^{\text {Mes }} / o^{\prime}-{ }^{\mathrm{P}} \mathrm{CH}_{3}{ }^{\text {Mes }}\right), 1.88 /$ $20.8\left(p-{ }^{\mathrm{P}} \mathrm{CH}_{3}{ }^{\text {Mes }} / p-{ }^{\mathrm{P}} \mathrm{CH}_{3}{ }^{\text {Mes }}\right), 1.74 / 20.6\left(o^{-}{ }^{\mathrm{C}} \mathrm{CH}_{3}{ }^{\text {Mes }} / o^{-}{ }^{\mathrm{C}} \mathrm{CH}_{3}{ }^{\text {Mes }}\right), 1.36 / 23.2\left(o_{-}{ }^{\mathrm{P}} \mathrm{CH}_{3}{ }^{\text {Mes }} / o^{-}{ }^{\mathrm{P}} \mathrm{CH}_{3}{ }^{\text {Mes }}\right)$, $-0.01 / 1.7\left(\mathrm{SiCH}_{3} / \mathrm{SiCH}_{3}\right)$.

${ }^{1} \mathrm{H}^{13}{ }^{13} \mathrm{C} \mathrm{GHMBC}\left(600 \mathrm{MHz} / 151 \mathrm{MHz}, \mathrm{C}_{6} \mathrm{D}_{6}, 299 \mathrm{~K}\right): \delta^{1} \mathrm{H} / \delta^{13} \mathrm{C}=7.35$ / 149.0, 137.1, 126.2 (5-H / C4, C8a, C7), 6.91 / 189.0, 140.5, 128.3 (8-H / C1, C4a, C6), 6.90 / 140.5, 126.5 (6-H / C4a, C8), 6.73 / 137.1,

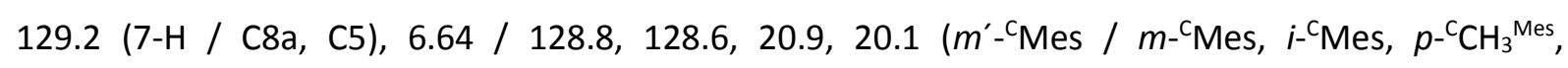
$\left.o^{\prime}-{ }^{\mathrm{C}} \mathrm{CH}_{3}{ }^{\text {Mes }}\right), 6.63$ / 129.9, 128.6, 20.9, 20.6 ( $m$ - ${ }^{\mathrm{C}}$ Mes / $m^{\prime}-{ }^{\mathrm{C}}$ Mes, $\left.i-{ }^{\mathrm{C}} \mathrm{Mes},{ }^{-}{ }^{\mathrm{C}} \mathrm{CH}_{3}{ }^{\text {Mes }}, o^{-}{ }^{\mathrm{C}} \mathrm{CH}_{3}{ }^{\text {Mes }}\right), 6.42$ / 131.5, 124.1, 20.8, 19.4 ( $m^{\prime}--^{\mathrm{P}}$ Mes / $m-{ }^{\mathrm{P}}$ Mes, $i-{ }^{\mathrm{P}}$ Mes, $\left.p-{ }^{\mathrm{P}} \mathrm{CH}_{3}{ }^{\mathrm{Mes}}, o^{\prime}-{ }^{\mathrm{P}} \mathrm{CH}_{3}{ }^{\mathrm{Mes}}\right), 6.34$ / 131.9, 124.1, 23.2, 20.8 (m- ${ }^{\mathrm{P}}$ Mes / $m^{\prime}-{ }^{\mathrm{P}}$ Mes, $i-{ }^{\mathrm{P}}$ Mes, $\left.0-{ }^{\mathrm{P}} \mathrm{CH}_{3}{ }^{\mathrm{Mes}}, p-{ }^{\mathrm{P}} \mathrm{CH}_{3}{ }^{\mathrm{Mes}}\right), 2.06 / 140.0,129.9,128.6\left(o^{\prime}-{ }^{\mathrm{C}} \mathrm{CH}_{3}{ }^{\mathrm{Mes}} /\right.$

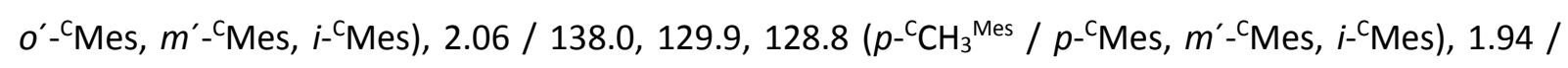

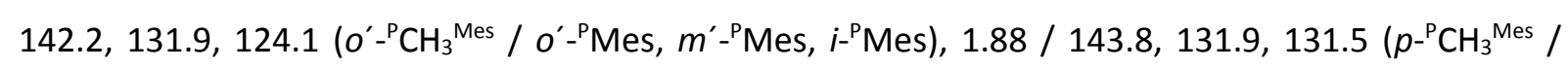
$p$ - ${ }^{\mathrm{P}}$ Mes, $m^{\prime}-{ }^{\mathrm{P}}$ Mes, $m-{ }^{\mathrm{P}}$ Mes), 1.74 / 139.8, 128.8, 128.6 (o- ${ }^{\mathrm{C}} \mathrm{CH}_{3}{ }^{\mathrm{Mes}} / o^{-}{ }^{\mathrm{C}}$ Mes, $m$ - ${ }^{\mathrm{C}}$ Mes, $i-{ }^{\mathrm{C}}$ Mes), 1.36 / 141.0, 131.5, 124.1 (o- $\left.{ }^{\mathrm{P}} \mathrm{CH}_{3}{ }^{\mathrm{Mes}} /{ }^{o-}{ }^{\mathrm{P}} \mathrm{Mes}, m^{-}{ }^{\mathrm{P}} \mathrm{Mes}, i^{\mathrm{P}} \mathrm{Mes}\right),-0.01 / 149.0,1.7\left(\mathrm{SiCH}_{3} / \mathrm{C} 4, \mathrm{SiCH}_{3}\right)$.

${ }^{19} \mathrm{~F},{ }^{19} \mathrm{~F}$ GCOSY (564 MHz / $564 \mathrm{MHz}, \mathrm{C}_{6} \mathrm{D}_{6}, 299 \mathrm{~K}$ ) [selected traces]: $\delta^{19} \mathrm{~F} / \delta^{19} \mathrm{~F}=-123.3 /-160.9$ $\left(o-\mathrm{PC}_{6} \mathrm{~F}_{5} / m-\mathrm{PC}_{6} \mathrm{~F}_{5}\right),-126.2 /-159.7\left(o^{\prime}-\mathrm{PC}_{6} \mathrm{~F}_{5} / m^{\prime}-\mathrm{PC}_{6} \mathrm{~F}_{5}\right),-138.3 /-164.1\left(o-\mathrm{C}_{6} \mathrm{~F}_{5} / m-\mathrm{C}_{6} \mathrm{~F}_{5}\right),-139.9 /$ $-168.0\left(o^{\prime}-\mathrm{C}_{6} \mathrm{~F}_{5} / m^{\prime}-\mathrm{C}_{6} \mathrm{~F}_{5}\right),-146.0 /-159.7,-160.9\left(p-\mathrm{PC}_{6} \mathrm{~F}_{5} / m^{\prime}-\mathrm{PC}_{6} \mathrm{~F}_{5}, m-\mathrm{PC}_{6} \mathrm{~F}_{5}\right),-158.1 /-163.6$ $\left(p-\mathrm{BC}_{6} \mathrm{~F}_{5} / m-\mathrm{BC}_{6} \mathrm{~F}_{5}\right),-159.2 /-164.1,-168.0\left(p-\mathrm{C}_{6} \mathrm{~F}_{5} / m-\mathrm{C}_{6} \mathrm{~F}_{5}, m^{\prime}-\mathrm{C}_{6} \mathrm{~F}_{5}\right)$. 


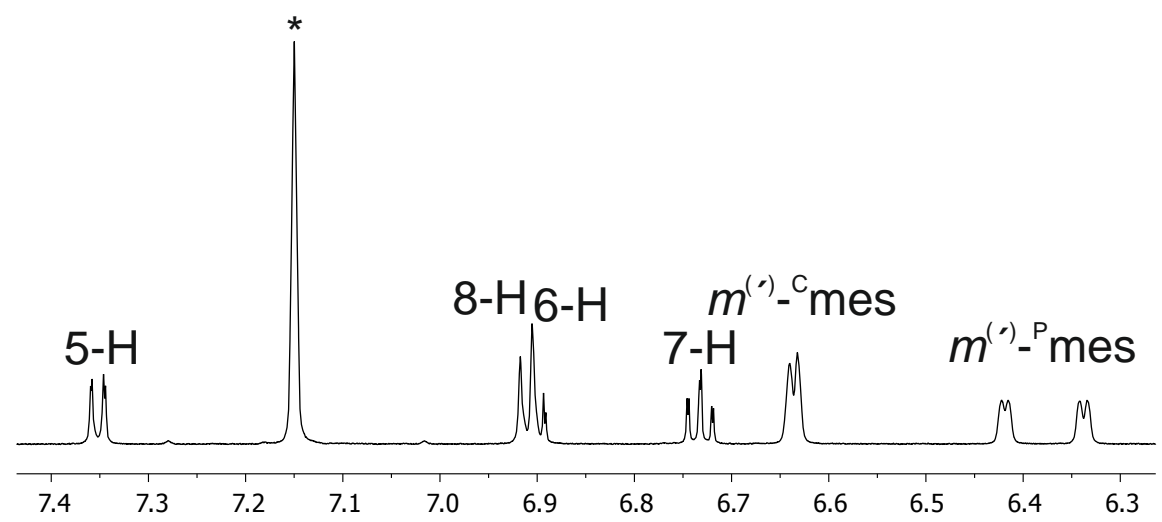

$\mathrm{SiCH}_{3}$

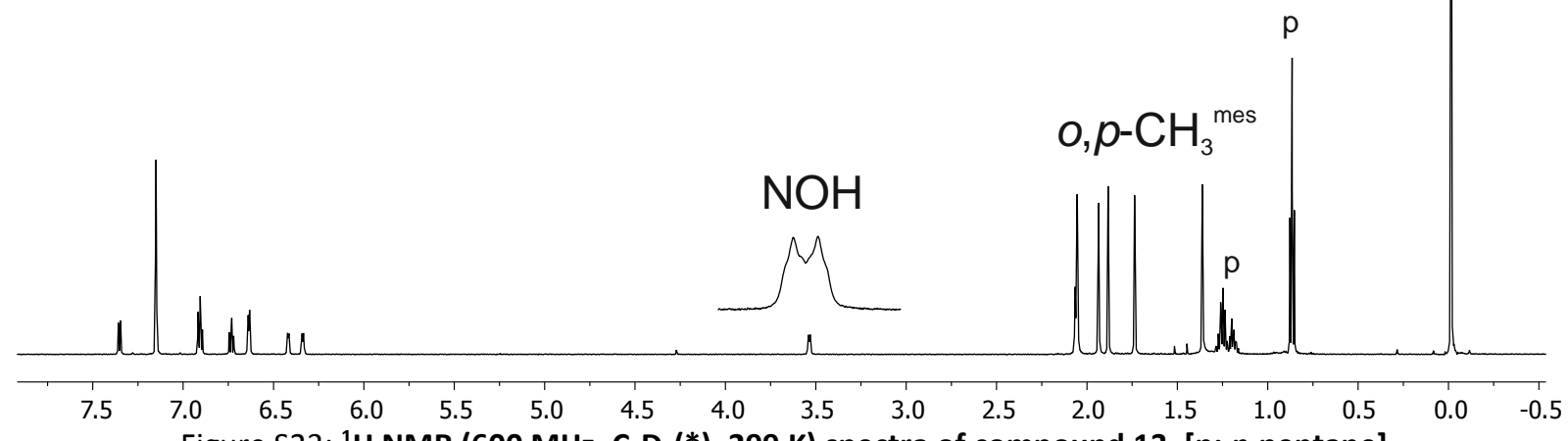

Figure S23: ${ }^{1} \mathrm{H}$ NMR (600 MHz, $\mathrm{C}_{6} \mathrm{D}_{6}(*)$ ), $299 \mathrm{~K}$ ) spectra of compound 13, [p: $n$-pentane].

${ }^{1} \mathrm{H}\left\{{ }^{31} \mathrm{P}\right\}$
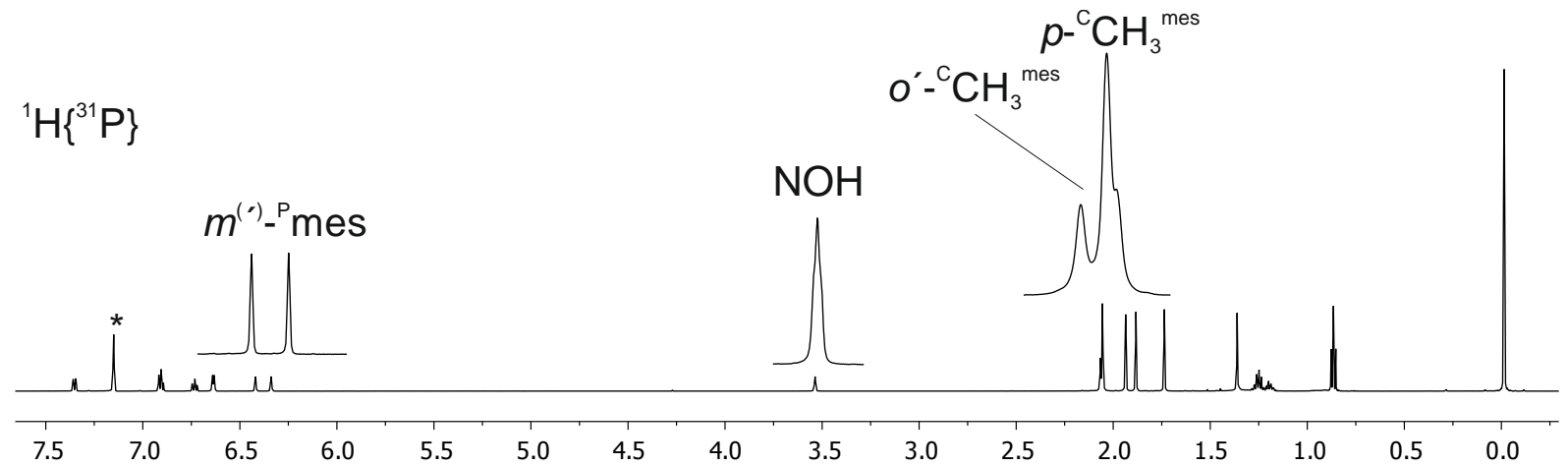

${ }^{1} \mathrm{H}$

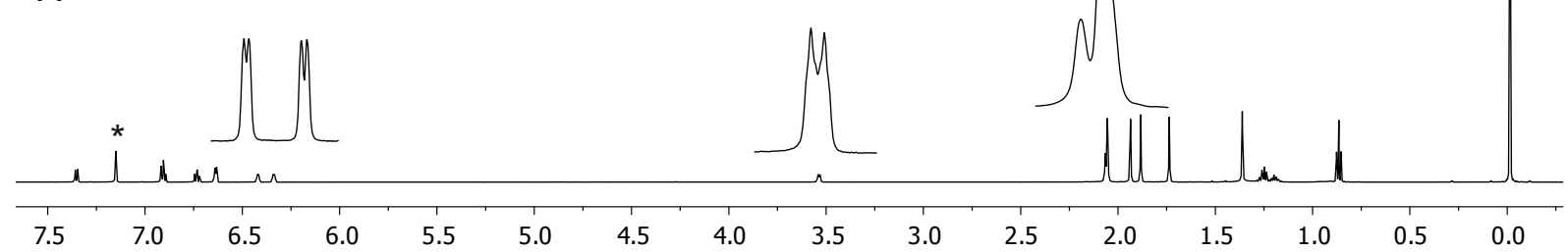
Figure S24: ${ }^{1} \mathrm{H}$ NMR (600 MHz, $\mathrm{C}_{6} \mathrm{D}_{6}\left({ }^{*}\right), 299 \mathrm{~K}$ ) and ${ }^{1} \mathrm{H}\left\{{ }^{31} \mathrm{P}\right\}$ NMR (600 MHz) spectra of compound 13. 

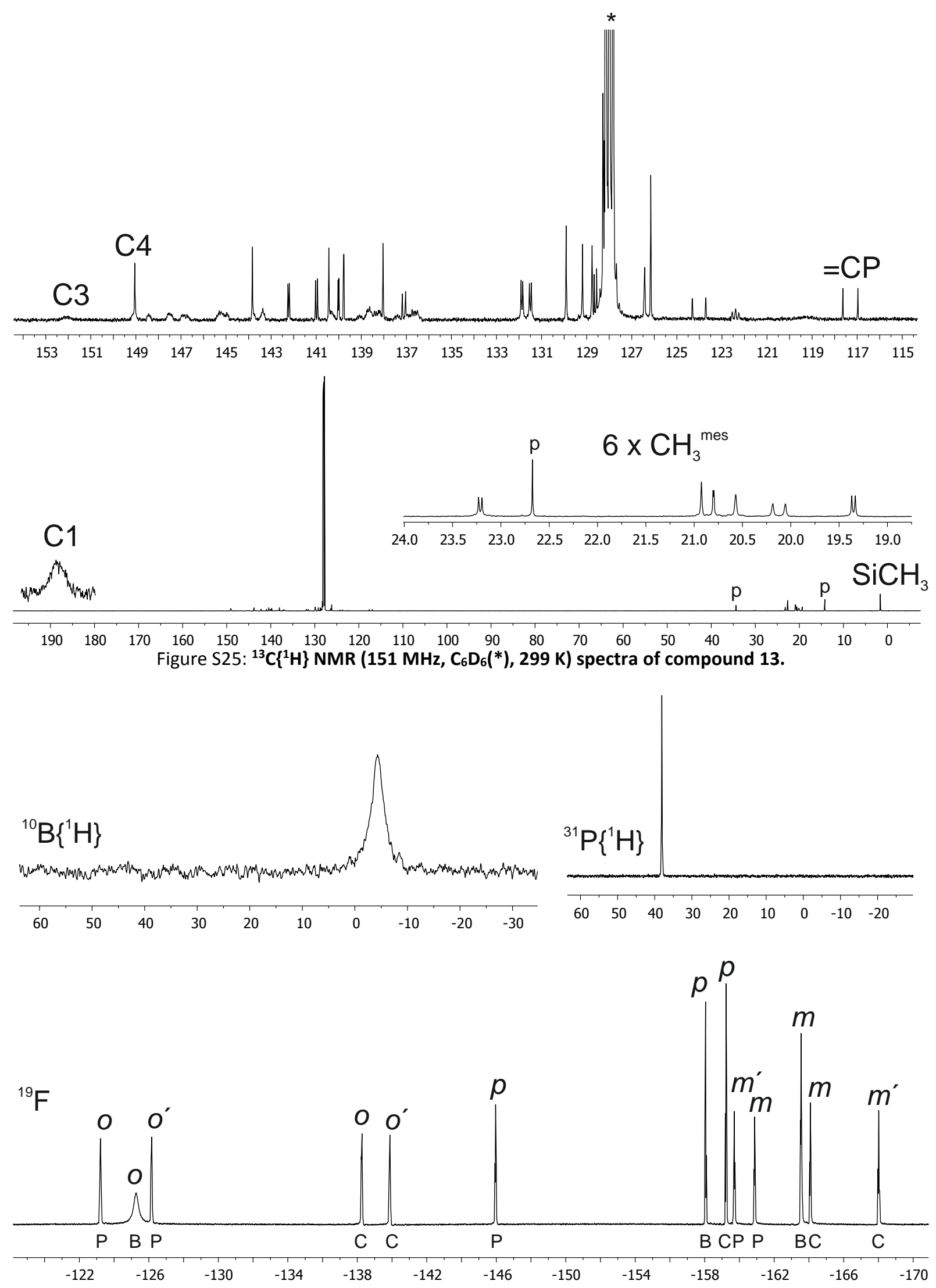

Figure S26: ${ }^{19} \mathrm{~F}$ NMR $\left(564 \mathrm{MHz}, \mathrm{C}_{6} \mathrm{D}_{6}, 299 \mathrm{~K}\right),{ }^{31} \mathrm{P}\left\{{ }^{1} \mathrm{H}\right\} \mathrm{NMR}(243 \mathrm{MHz})$ and ${ }^{10} \mathrm{~B}\left\{{ }^{1} \mathrm{H}\right\} \mathrm{NMR}(64 \mathrm{MHz})$ spectra of compound 13. 
Comment: please note, in the ${ }^{31} \mathrm{P},{ }^{1} \mathrm{H}$ ghmbc experiment the ${ }^{1} \mathrm{H}$ resonances of the para- and orthomethyl group of the carbon bonded mesityl group generate a cross peaks to the ${ }^{31} \mathrm{P}$.

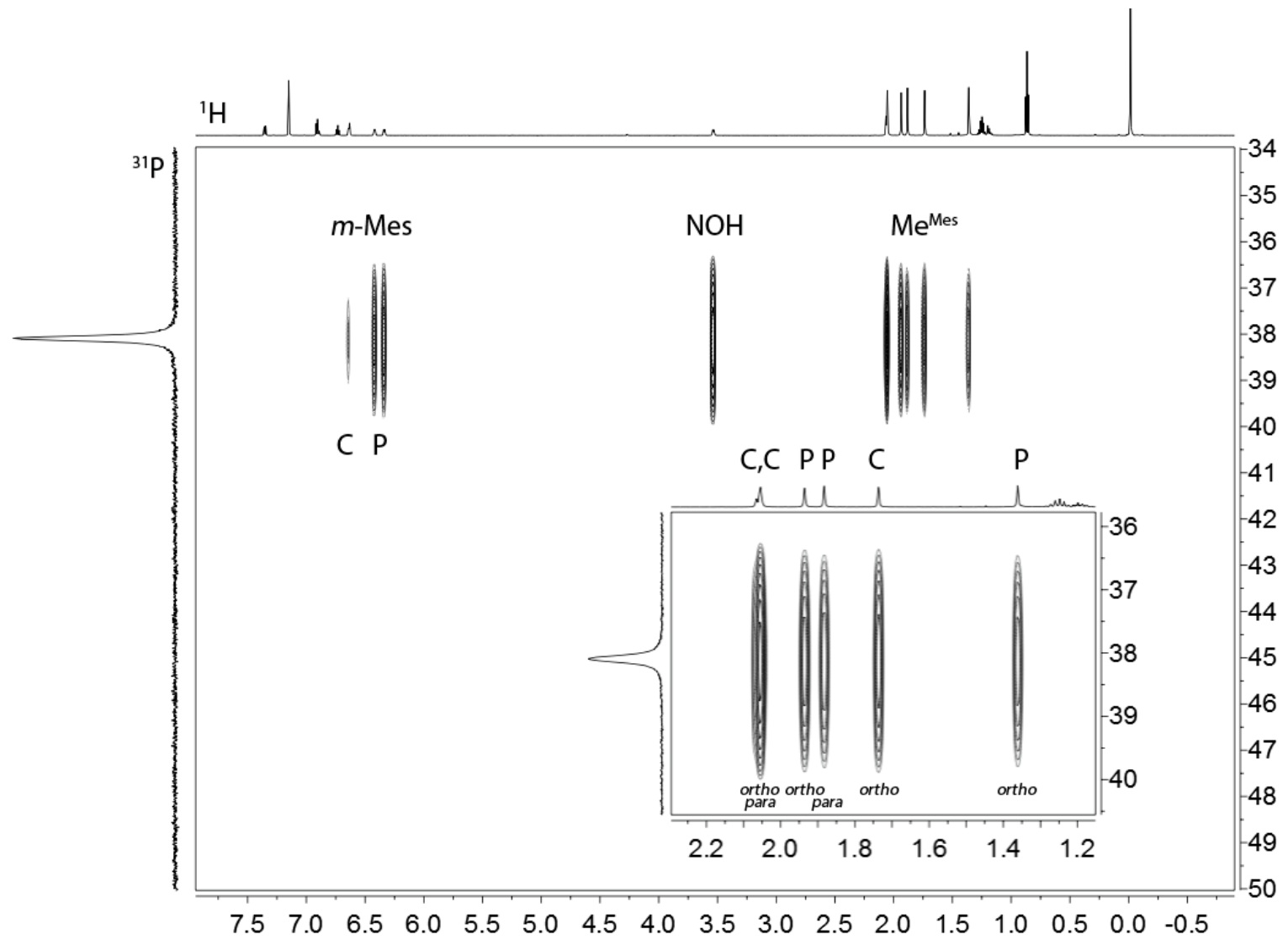

Figure S27: ${ }^{31} \mathrm{P},{ }^{1} \mathrm{H}$ ghmbc $\left(600,243 \mathrm{MHz}, \mathrm{C}_{6} \mathrm{D}_{6}, 299 \mathrm{~K}\right)$ spectra of compound 13

Figure S28: X-ray crystal structure analysis of compound 13: formula $\mathrm{C}_{49} \mathrm{H}_{36} \mathrm{BF}_{15} \mathrm{NOPSi} \cdot \mathrm{C}_{6} \mathrm{H}_{6}, M=$ 1087.77, colourless crystal, $0.22 \times 0.05 \times 0.02 \mathrm{~mm}, a=30.7946(5), b=17.3685(3), c=19.8227(3) \AA, V$ $=10602.3(3) \AA^{3}, \rho_{\text {calc }}=1.363 \mathrm{gcm}^{-3}, \mu=1.495 \mathrm{~mm}^{-1}$, empirical absorption correction $(0.734 \leq \mathrm{T} \leq$ 0.970), $Z=8$, orthorhombic, space group $P b c n$ (No. 60), $\lambda=1.54178 \AA, T=223(2) K, \omega$ and $\phi$ scans, 57133 reflections collected $( \pm h, \pm k, \pm /), 8511$ independent $\left(R_{\text {int }}=0.097\right)$ and 5096 observed reflections $[/>2 \sigma(I)], 674$ refined parameters, $R=0.059, w R^{2}=0.171$, max. (min.) residual electron density 0.21 $(-0.40)$ e. $\AA^{-3}$, hydrogen atoms were calculated and refined as riding atoms. 


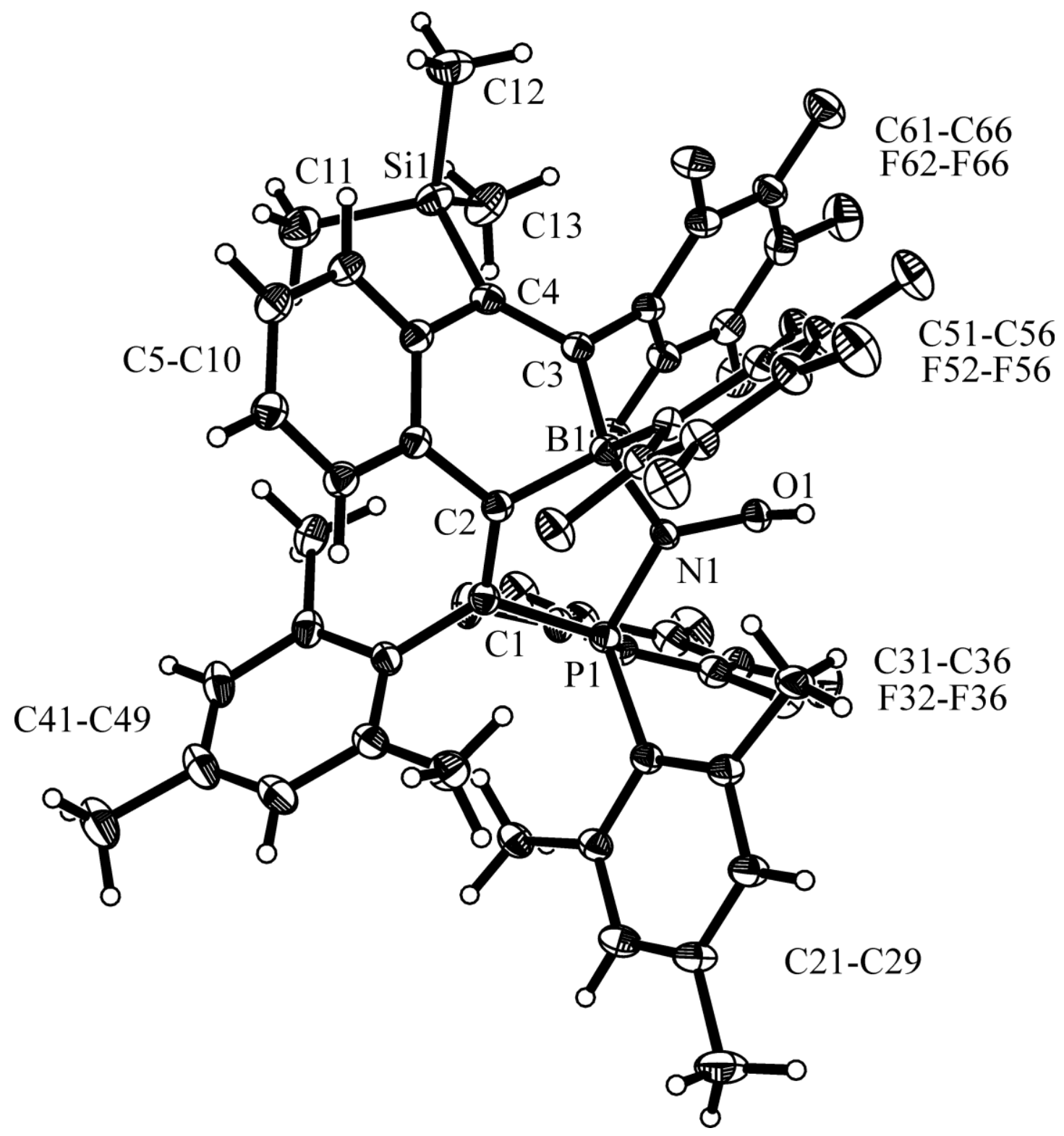




\section{Synthesis of the diastereomers 14/14'}

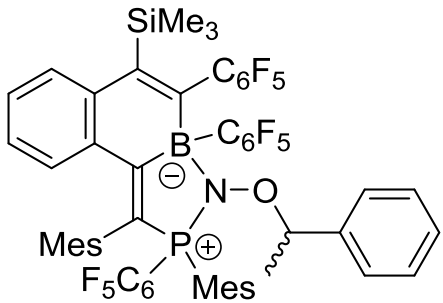

Scheme S9

A flask with compound 12 (101 mg, $0.1 \mathrm{mmol}, 1 \mathrm{eq}$ ), Cu-powder (11.0 mg, $\quad 0.17 \mathrm{mmol}, \quad 1.7 \mathrm{eq}), \quad$ 5,5'-di-tert-butyl-2,2'-bipyridine (11.0 mg, $0.04 \mathrm{mmol}, 0.4 \mathrm{eq})$ and $\mathrm{Cu}(\mathrm{OTf})_{2}(3.0 \mathrm{mg}, 0.01 \mathrm{mmol}, 0.1 \mathrm{eq})$ were suspended in $\mathrm{C}_{6} \mathrm{H}_{6}(\sim 2 \mathrm{~mL})$ and 1-bromoethylbenzene was added. The resulting suspension was degassed by pump-freeze-thaw cycles $(3 \mathrm{x})$ and then it was heated to $70{ }^{\circ} \mathrm{C}$ for 2 days. It was cooled to room temperature and the supernatant was collected using a pipette. The residue was washed with $\mathrm{C}_{6} \mathrm{H}_{6}(\sim 2 \mathrm{~mL})$ and the solvent of the combined organic phases was removed in vacuo. The residue was purified by flash-chromatography $\left(\mathrm{SiO}_{2}, n\right.$-pentane : $\left.\mathrm{CH}_{2} \mathrm{Cl}_{2}=4: 1\right)$ to give a solid $(30.0 \mathrm{mg}$, $0.03 \mathrm{mmol}, 27 \%)$.

IR (ATR): $\tilde{v}\left[\mathrm{~cm}^{-1}\right]=3543(\mathrm{w}), 2956$ (br m), 2926 (br m), $1643(\mathrm{~m}), 1606(\mathrm{~m}), 1516(\mathrm{~s}), 1485$ (s), 1455 (s), $1383(w), 1300(w), 1253(m), 1150(w), 1100(s), 1016(m), 984(s), 917(m), 845(s), 768(m), 685$ $(w), 656(m), 604(w), 514(w)$.

Elemental analysis: Calcd for $\mathrm{C}_{57} \mathrm{H}_{44} \mathrm{BF}_{15} \mathrm{NOPSi}: \mathrm{C}, 61.47 \mathrm{H}, 3.98 \mathrm{~N}, 1.26$. Found: $\mathrm{C}, 61.74 \mathrm{H}, 4.04 \mathrm{~N}$, 1.14 .

Decomposition (DSC): $210^{\circ} \mathrm{C}$.

NMR characterization of the obtained solid in $C_{6} D_{6}$ showed a mixture of diastereomers $(\sim 61: 39$ mol\% $\left.\left({ }^{1} \mathrm{H}\right)\right)$, which were identified by selected NMR resonances.

${ }^{1} \mathrm{H}$ NMR (600 MHz, $\mathrm{C}_{6} \mathrm{D}_{6}, 299 \mathrm{~K}$ ) [selected resonances]: $\delta$ (major) $=4.71(\mathrm{~m}, 1 \mathrm{H}, \mathrm{OCH}), 0.01(\mathrm{~s}, 9 \mathrm{H}$, $\left.\mathrm{SiCH}_{3}\right) . \delta$ (minor) $=4.82(\mathrm{~m}, 1 \mathrm{H}, \mathrm{OCH}),-0.06\left(\mathrm{~s}, 9 \mathrm{H}, \mathrm{SiCH}_{3}\right)$.

${ }^{13} \mathrm{C}\left\{{ }^{1} \mathrm{H}\right\}$ NMR (151 MHz, $\mathrm{C}_{6} \mathrm{D}_{6}, 299 \mathrm{~K}$ ) [selected resonances]: $\delta$ (major) = $191.0\left(\mathrm{C} 1^{\mathrm{a}, \mathrm{b}}\right.$ ), 150.3 (C4), 83.7 (br, OCH), $1.75\left(\mathrm{SiCH}_{3}\right) . \delta$ (minor) = $191.0\left(\mathrm{Cl}^{\mathrm{a}, \mathrm{b}}\right), 149.2(\mathrm{C} 4), 83.2(\mathrm{br}, \mathrm{OCH}), 1.76\left(\mathrm{SiCH}_{3}\right)$.

${ }^{31} \mathrm{P}\left\{{ }^{1} \mathrm{H}\right\}$ NMR (243 MHz, $\left.\mathrm{C}_{6} \mathrm{D}_{6}, 299 \mathrm{~K}\right): \delta=52.7\left(\mathrm{v}_{1 / 2} \sim 180 \mathrm{~Hz}, 35 \mathrm{mo} \%\right), 48.3\left(\mathrm{v}_{1 / 2} \sim 30 \mathrm{~Hz}, 65 \mathrm{~mol} \%\right)$. ${ }^{10} \mathrm{~B}\left\{{ }^{1} \mathrm{H}\right\} \mathrm{NMR}\left(64 \mathrm{MHz}, \mathrm{C}_{6} \mathrm{D}_{6}, 299 \mathrm{~K}\right): \delta=-2.3\left(\mathrm{v}_{1 / 2} \sim 250 \mathrm{~Hz}\right)$.

${ }^{29} \mathrm{Si}\left\{{ }^{1} \mathrm{H}\right\}$ DEPT (119 MHz, $\left.\mathrm{C}_{6} \mathrm{D}_{6}, 299 \mathrm{~K}\right): \delta=-5.7\left(\mathrm{v}_{1 / 2} \sim 2 \mathrm{~Hz}\right.$, major), $-6.0\left(\mathrm{v}_{1 / 2} \sim 2 \mathrm{~Hz}\right.$, minor). 

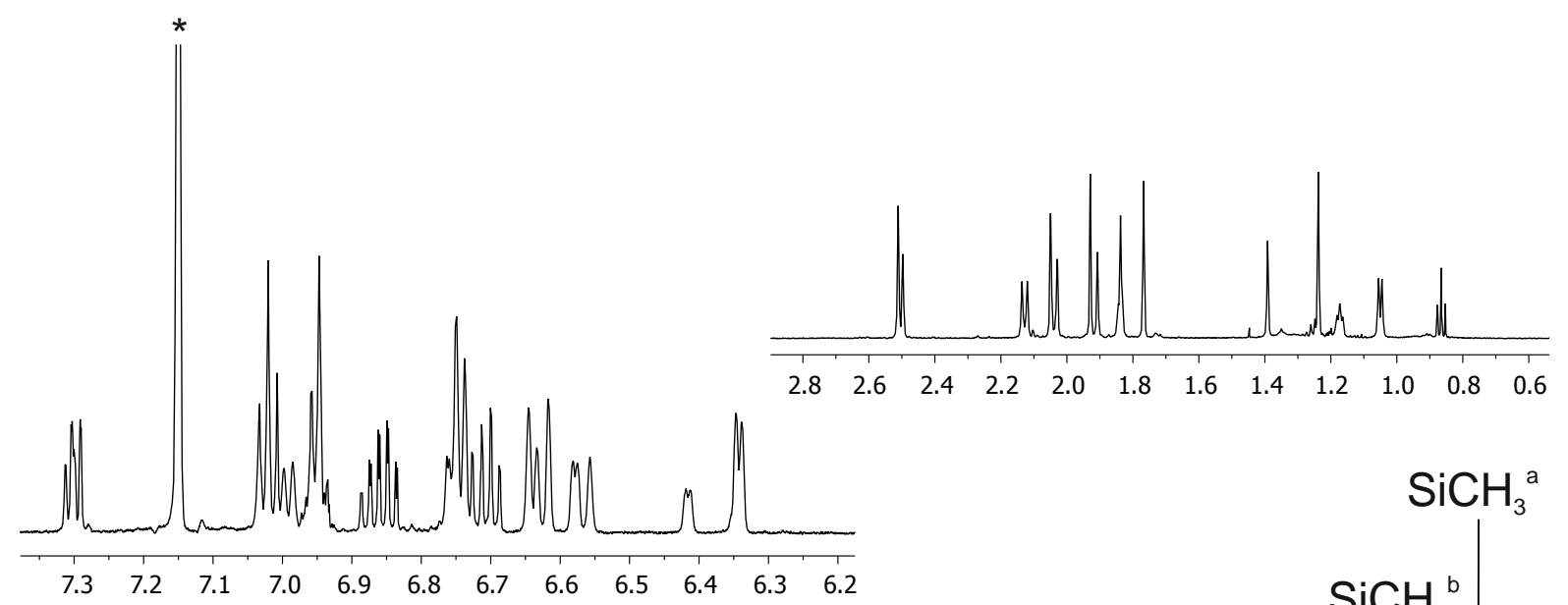

$\mathrm{OCH}^{\mathrm{a}}$
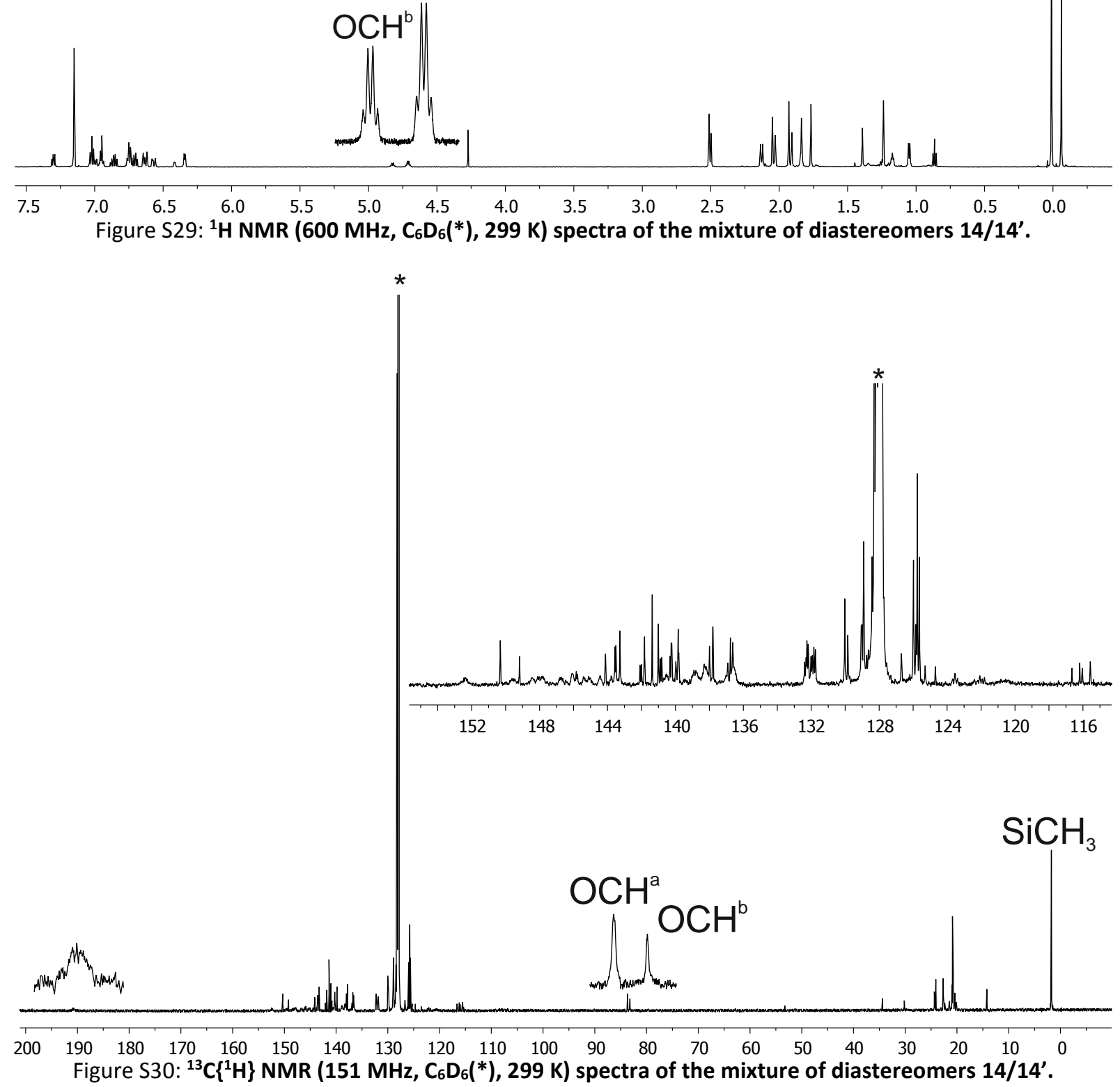

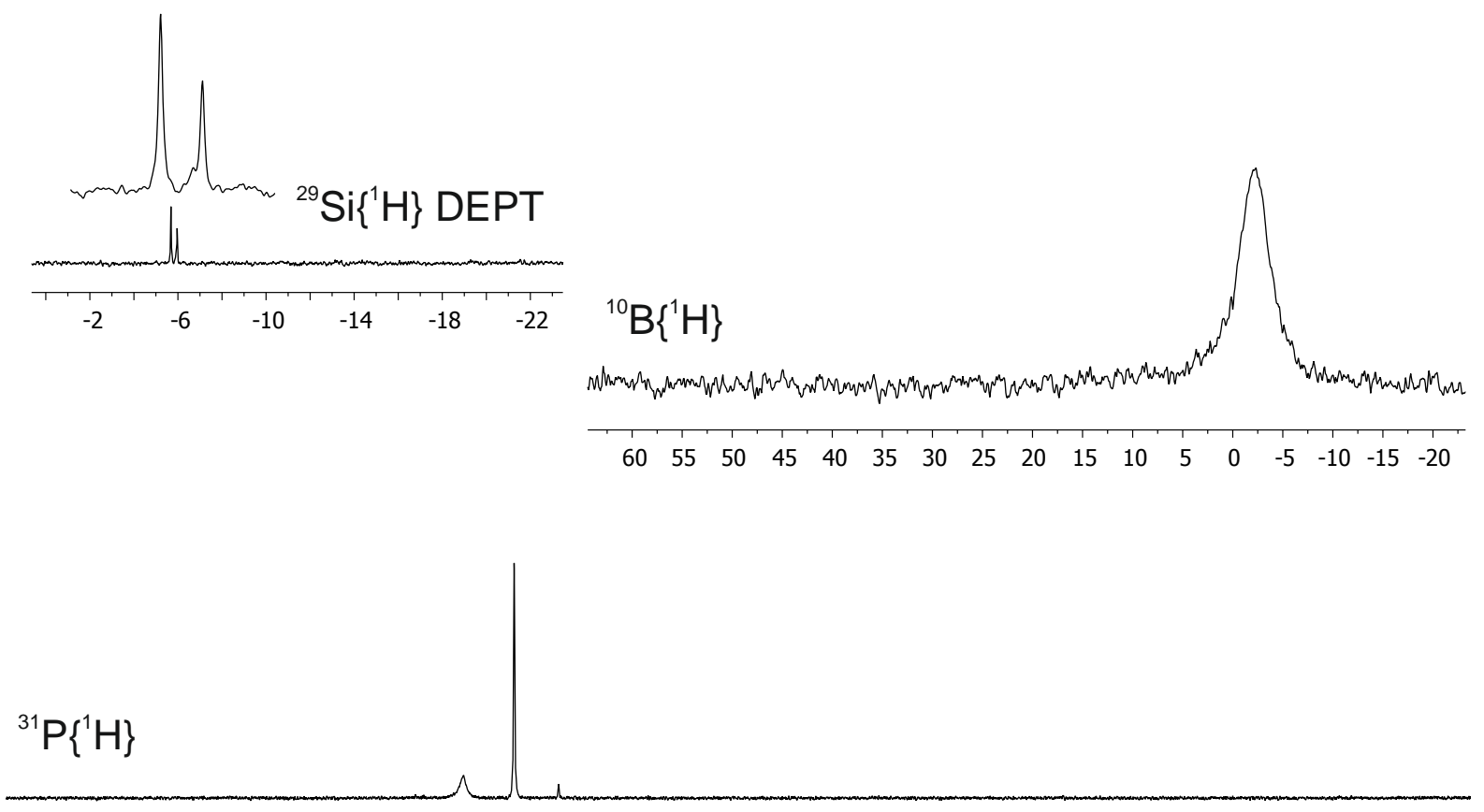

$\begin{array}{lllllllllllllllllllllllllllll}90 & 85 & 80 & 75 & 70 & 65 & 60 & 55 & 50 & 45 & 40 & 35 & 30 & 25 & 20 & 15 & 10 & 5 & 0 & -5 & -10 & -15 & -20 & -25 & -30 & -35\end{array}$ Figure S31: ${ }^{31} \mathrm{P}\left\{{ }^{1} \mathrm{H}\right\}$ NMR (243 MHz, $\left.\mathrm{C}_{6} \mathrm{D}_{6}, 299 \mathrm{~K}\right),{ }^{10} \mathrm{~B}\left\{{ }^{1} \mathrm{H}\right\}$ NMR (64 MHz) and ${ }^{29} \mathrm{Si}\left\{{ }^{1} \mathrm{H}\right\}$ DEPT (119 MHz) spectra of the mixture of diastereomers 14/14'

Single crystals suitable for the $X$-ray crystal structure analysis (two diastereomers: rac- $R^{P}, R^{C}, S^{B}(85 \%)$ and rac- $\left.R^{P}, S^{C}, S^{B}(15 \%)\right)$ were obtained from a solution of the obtained solid in $\mathrm{C}_{6} \mathrm{H}_{6}$ at room temperature.

Figure S32: X-ray crystal structure analysis of compound 14: formula $\mathrm{C}_{57} \mathrm{H}_{44} \mathrm{BF}_{15} \mathrm{NOPSi} \cdot \mathrm{C}_{6} \mathrm{H}_{6}, M=$ 1191.91, colourless crystal, $0.15 \times 0.08 \times 0.02 \mathrm{~mm}, a=11.7505(4), b=12.4955(5), c=21.0100(12) \AA$, $\alpha=102.451(3), 6=92.353(3), \gamma=110.290(2)^{\circ}, V=2802.7(2) \AA^{3}, \rho_{\text {calc }}=1.412 \mathrm{gcm}^{-3}, \mu=0.165 \mathrm{~mm}^{-1}$, empirical absorption correction $(0.975 \leq T \leq 0.996), Z=2$, triclinic, space group $P \overline{1}$ (No. 2$), \lambda=$ $0.71073 \AA, T=223(2) \mathrm{K}, \omega$ and $\phi$ scans, 26068 reflections collected $( \pm h, \pm k, \pm /), 9463$ independent $\left(R_{\text {int }}=0.057\right)$ and 6816 observed reflections $[/>2 \sigma(I)], 878$ refined parameters, $R=0.063, w R^{2}=0.155$, max. (min.) residual electron density $0.27(-0.25)$ e. $\AA^{-3}$, hydrogen atoms were calculated and refined as riding atoms. 

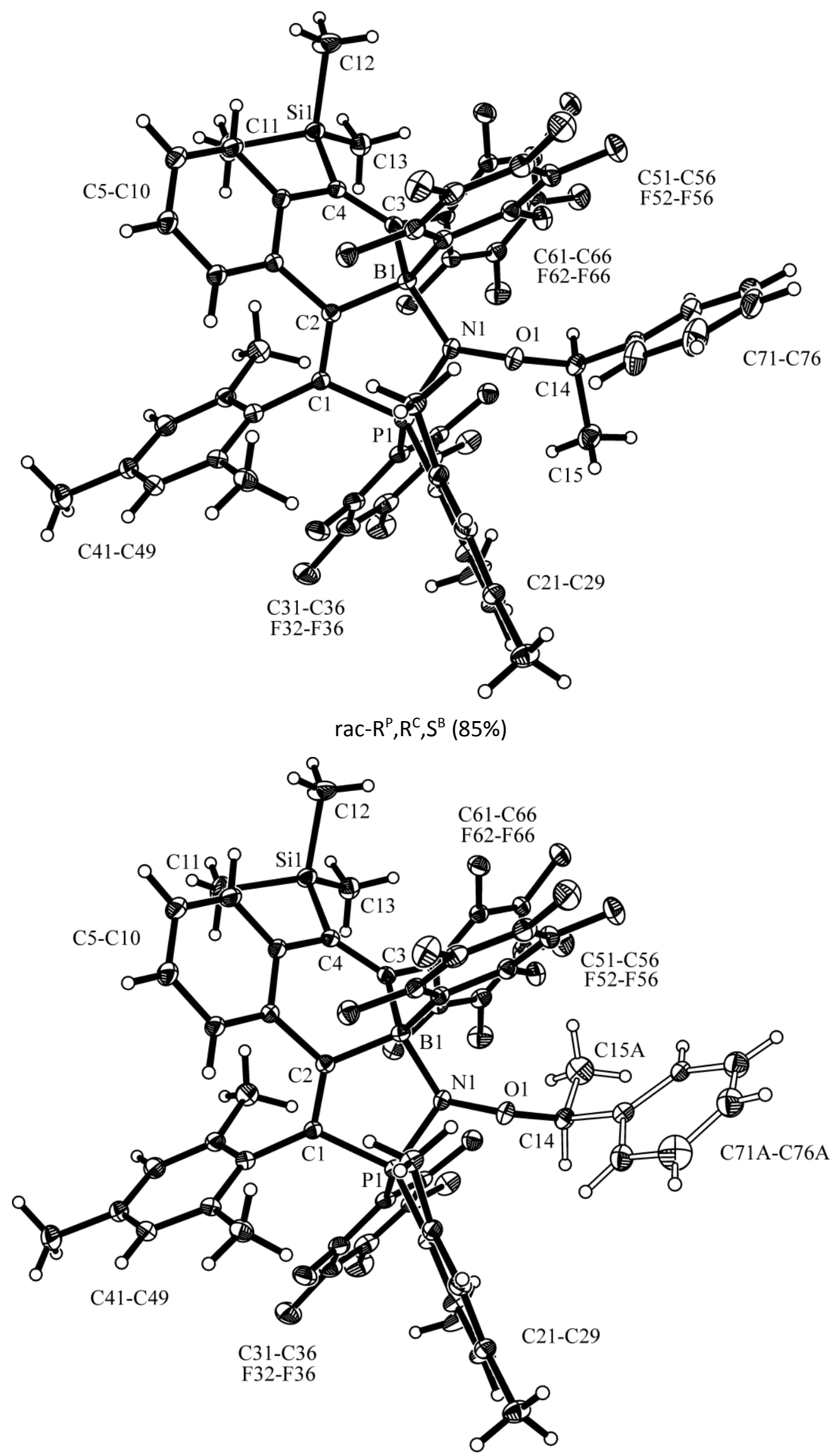

$\operatorname{rac}-R^{P}, S^{C}, S^{B}(15 \%)$ 


\section{Polymerzation reactions:}

General (solvents, reagents and equipment used): Styrene was freshly distilled over $\mathrm{CaH}_{2}$. Benzene was freshly distilled over Na. Gel permeation chromatography (GPC) was carried out with degassed THF as eluent at a flow rate of $1.0 \mathrm{~mL} / \mathrm{min}$ at $25^{\circ} \mathrm{C}$ on a system consisting of an HPLC Pump 64 (Knauer), a set of two PLgel $5 \mu \mathrm{m}$ MIXED-C columns (300 $\times 7.5 \mathrm{~mm}$, Polymer Laboratories) and a Shodex RI differential refractometer detector. Data were analyzed with PSS WinGPC Compact V.7.20 software (Polymer Standards Service) based on calibration curves built upon poly(styrene) standards (Polymer Laboratories Poly(styrene) Medium MW Calibration Kit to determine the molecular weight of polymers) with peak molecular weights ranging from 1660 to $1000000 \mathrm{~g} / \mathrm{mol}$.

General Procedure for the Polymerization of Styrene with Alkoxyamines 14/14': A heat gun dried Schlenk tube was charged with alkoxyamines 14/14' (10 mg, $1.0 \mathrm{~mol} \%, 1.0 \mathrm{~mol} \%$ ), free nitroxide 12 (0-1.7 mol\%), styrene (94 mg, $0.90 \mathrm{mmol}, 1.0$ eq.) and benzene $(0.20 \mathrm{~mL})$ under argon. The tube was subjected to three freeze-thaw cycles and then sealed. The polymerization was carried out at 90 $110^{\circ} \mathrm{C}$ for 8-11 h. After cooling to ambient temperature, the solvent and residual monomer were removed under reduced pressure. The polymer was dissolved in DCM $(0.2 \mathrm{~mL})$ and precipitated into $\mathrm{MeOH}(5 \mathrm{~mL}$ ) three times. The product was dried in vacuo and the target polymer was obtained as white powder. Yield was determined gravimetrically, molecular weight and PDI were determined by GPC.

Polymerization of Styrene with Alkoxyamines 14/14': The diastereoisomers 14/14' were also tested as initiators/regulators for the nitroxide mediated radical polymerization (NMP) ${ }^{[1]}$ of styrene (Table S1). Polymerizations were conducted using $\mathbf{1 4 / \mathbf { 1 4 } ^ { \prime }}$ as a $\mathbf{1 : 1}$ mixture of diastereoisomers in benzene with 1.0 mol\% of $14 / 14^{\prime}$ with respect to styrene at $90^{\circ} \mathrm{C}$ for $9 \mathrm{~h}$ under inert gas atmosphere (Table S1, entry 1). Under these conditions polymerization did not occur. Running the same experiment at $100^{\circ} \mathrm{C}$ for $8 \mathrm{~h}$ provided poly(styrene) in $11 \%$ yield (after precipitation from methanol, entry 2). Gel permeation chromatography (GPC) revealed an average molecular weight of $M_{n}=69.6 \mathrm{kDa}$ and a polydispersity index (PDI) of 1.67 . Polymerization at $110^{\circ} \mathrm{C}$ under otherwise identical conditions provided polystyrene in $25 \%$ yield with $M_{n}$ of $59.2 \mathrm{kDa}$ and a PDI of 1.57 (entry 3). In an attempt to increase the controlled character of the radical polymerization, free nitroxide 12 (1.7 mol\%) was added to the polymerization. As expected, average molecular weight decreased $\left(M_{n}=11800 \mathrm{kDa}\right)$, however, PDI (1.88) remained large indicating little or no control over the polymerization (entry 4). Notably, styrene autopolymerization at $110^{\circ} \mathrm{C}$ provided polystyrene with significantly larger $\mathrm{Mn}$ (entry 5), revealing that alkoxyamines $\mathbf{1 4} / \mathbf{1 4}^{\prime}$ are capable of mediating the polymerization of styrene to some extent. However, efficiency of these alkoxyamines as NMP initiators/regulators is very low. 
Table S1: Polymerisation data of styrene with the alkoxyamines $14 / 14$

\begin{tabular}{ccccccc} 
Entry & $\mathbf{T}\left[{ }^{\circ} \mathbf{C}\right]$ & $\mathbf{1 2}[\mathbf{m o l} \%]$ & $\mathbf{t ~ [ h ]}$ & yield [\%] & $\mathbf{M}_{\mathbf{n}}[\mathbf{k D a}]$ & PDI \\
\hline $\mathbf{1}$ & 90 & - & 9 & 0 & - & - \\
$\mathbf{2}$ & 100 & - & 8 & 11 & 69.6 & 1.67 \\
$\mathbf{3}$ & 110 & - & 8 & 25 & 59.2 & 1.57 \\
$\mathbf{4}$ & 110 & 1.7 & 11 & 27 & 11.8 & 1.88 \\
$\mathbf{5}^{*}$ & 110 & - & 5 & 23 & 255.0 & 1.70 \\
& *Experiment was conducted without initiator in neat styrene.
\end{tabular}

Ref. [1]: (a) Hawker, C. J.; Bosman, A. W.; Harth, E. Chem. Rev. 2001, 101, 3661-3688. (b) Sciannamea, V.; Jérôme, R.; Detrembleur, C. Chem. Rev. 2008, 108, 1104-1126. 